Purdue University

Purdue e-Pubs

Open Access Theses

Theses and Dissertations

Summer 2014

\title{
Search for new physical phenomena via displaced muon signatures with the CMS detector at the LHC
}

Melih Solmaz

Purdue University

Follow this and additional works at: https://docs.lib.purdue.edu/open_access_theses

Part of the Elementary Particles and Fields and String Theory Commons

\section{Recommended Citation}

Solmaz, Melih, "Search for new physical phenomena via displaced muon signatures with the CMS detector at the LHC" (2014). Open Access Theses. 688.

https://docs.lib.purdue.edu/open_access_theses/688

This document has been made available through Purdue e-Pubs, a service of the Purdue University Libraries. Please contact epubs@purdue.edu for additional information. 
Graduate School ETD Form 9

(Revised 01/14)

\section{PURDUE UNIVERSITY \\ GRADUATE SCHOOL \\ Thesis/Dissertation Acceptance}

This is to certify that the thesis/dissertation prepared

By Melih Solmaz

Entitled

SEARCH FOR NEW PHYSICAL PHENOMENA VIA DISPLACED MUON SIGNATURES WITH

THE CMS DETECTOR AT THE LHC

For the degree of $\quad$ Master of Science

Is approved by the final examining committee:

Ian P. Shipsey

Daniela Bortoletto

Martin Kruczenski

To the best of my knowledge and as understood by the student in the Thesis/Dissertation Agreement. Publication Delay, and Certification/Disclaimer (Graduate School Form 32), this thesis/dissertation adheres to the provisions of Purdue University's "Policy on Integrity in Research" and the use of copyrighted material.

Ian P. Shipsey

Approved by Major Professor(s):

Approved by: Marc W. Caffee $07 / 30 / 2014$

Head of the Department Graduate Program

Date 


\title{
SEARCH FOR NEW PHYSICAL PHENOMENA VIA DISPLACED MUON SIGNATURES WITH THE CMS DETECTOR AT THE LHC
}

\author{
A Thesis \\ Submitted to the Faculty \\ of \\ Purdue University \\ by \\ Melih Solmaz
}

In Partial Fulfillment of the

Requirements for the Degree

of

Master of Science

August 2014

Purdue University

West Lafayette, Indiana 
À mon frère et maman, la plus belle du monde 
"When spring comes, If I'm already dead, The flowers will blossom the same way, And the trees will be no greener than last spring. Reality needs me not. I feel great joy In considering that my death is not important at all." -Fernando Pessoa- 


\section{ACKNOWLEDGMENTS}

Looking back at last two years, meeting my supervisor, Ian Shipsey, has been one of the turning points in my life for sure, though describing him just as my supervisor would be an understatement. Following his advice, I attended CMS Data Analysis School at Fermilab in January 2013 and have been actively taking part in the same experiment ever since. In other words, CMS has been a part of me.

When I started to work in the Purdue CMS Exotica group, there was the idea of extending the search for long-lived beyond Standard Model particles at the LHC by using only the muon chambers. I was thrilled by the idea because it was original. I was then encouraged to work on this research subject and I went for it. To make the long story shorter, it has been no bed of roses yet a fantastic learning experience in a friendly research environment.

I would like to express my deepest appreciation to Ian Shipsey for believing in me and truly caring about my development and future as a young researcher. He has meant more than an advisor to me. The vision he gave me as a scientist will never abandon me as time goes by. I am also grateful that he made all the facilitative arrangements at Purdue to keep me focused on the research despite his move to Oxford. Without his guidance, wisdom and persistent help this thesis would not have been possible.

In addition, I would like to thank also to my thesis committee members, Daniela Bortoletto and Martin Kruczenski for raising exceptional questions about the analysis, some of which are discussed in the text.

It has been my sincere privilege to work with Marco De Mattia, Zhen $\mathrm{Hu}$ and Nuno Leonardo. I frequently benefited from their research experience and broad

physics knowledge throughout my research at Purdue. I am greatly indebted to them 
for the preparation of this thesis. It is unfortunate that this successful group has to disintegrate soon.

I would like to give special thanks to Denes Molnar for his support during my graduate school applications. The informal discussions I had with him were unforgettable and broadened my horizons. Words can not describe how thankful I am to Sandy Formica for being not only the greatest graduate secretary ever but also a person with an understanding heart saving me from lots of trouble.

Sandra Sweval, another genuine Midwest lady, thank you very much for letting me practice my flute in the sanctuary of the University Church. It was stress reliever for me. Mariko Knox, please accept my gratitude for taking your time to teach me the fundamentals of the Argentinian Tango patiently. Domo arigato! James Foster, I thank you from the bottom of my heart for all intellectually stimulating conversations about life, existence, love and literature. I lost the track of time in the course of these discussions at Greyhouse. Thank you all. 


\section{TABLE OF CONTENTS}

Page

LIST OF TABLES . . . . . . . . . . . . . . . . . . . v viii

LIST OF FIGURES . . . . . . . . . . . . . . . . .

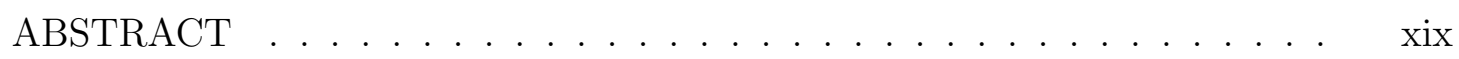

1 Introduction . . . . . . . . . . . . . . . . . . . . 1

2 The CMS detector . . . . . . . . . . . . . . . . . 4

3 Data and Monte Carlo simulation samples . . . . . . . . . . . 8

4 Event reconstruction and selection . . . . . . . . . . . . . . . 12

4.1 Displaced track reconstruction . . . . . . . . . . . . . . . . 12

4.2 Muon selection . . . . . . . . . . . . . . . . . . 12

4.3 Selection of long-lived exotica . . . . . . . . . . . . . . . . . . . . . . . . . . . . . . . . .

4.4 Results of blind analysis . . . . . . . . . . . . . . . . 17

4.5 Selection efficiency and acceptance . . . . . . . . . . . . 20

5 Estimated background and associated systematic uncertainties . . . . . . 28

5.1 Background validation . . . . . . . . . . . . . . . 29

5.2 Background systematic uncertainties . . . . . . . . . . . 30

6 Signal systematic uncertainties . . . . . . . . . . . . . . . . . . 33

6.1 Luminosity . . . . . . . . . . . . . . . . . . . . . . 33

6.2 Effect of pileup . . . . . . . . . . . . . . . . . . . . 34

6.3 Track finding and selection efficiency . . . . . . . . . . . . . . . . 34

6.4 Trigger efficiency measurement . . . . . . . . . . . . . . . 47

6.5 Effect of the $p_{\mathrm{T}}$ resolution . . . . . . . . . . . . . . . . 52

7 Analysis sensitivity and expected upper limits . . . . . . . . . . . . 53

7.1 Comparison with the tracker-based analysis . . . . . . . . . . 54

7.2 Combined limits . . . . . . . . . . . . . . . . . . . 58

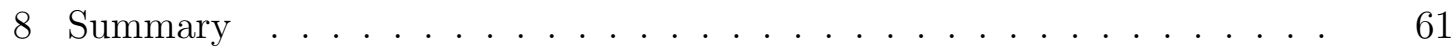

REFERENCES . . . . . . . . . . . . . . . . . . 62 APPENDICES

A Performance of refittedStandAlone muons . . . . . . . . . . . . . . . 64

A.1 Track parameters of RSA and SA muons . . . . . . . . . . 65 
A.2 Reconstruction efficiencies of prompt and displaced muons . . . . . 71

B Muon timing information in the events removed by cosmic rejection . . . 73

C Muon trigger efficiency . . . . . . . . . . . . . . . . 76

D Agreement between data and the background simulation . . . . . . 87

E Effect of the RSA muon $p_{\mathrm{T}}$ resolution on the analysis . . . . . . . . . . . 91

F $\quad L_{x y}$ resolution of RSA muons for prompt decays . . . . . . . . . . . 95

G Secondary vertex reconstruction efficiency f . . . . . . . . . . . 98

G.1 Secondary vertex reconstruction efficiency in signal MC . . . . . . . 98

G.2 Secondary vertex reconstruction efficiency in cosmics data and simula-

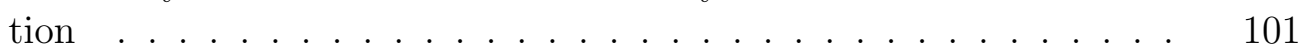

$\mathrm{H}$ Alternative background systematics estimate . . . . . . . . . . 103 


\section{LIST OF TABLES}

Table

Page

3.1 The CMS datasets used by the analysis. . . . . . . . . . . . . . 8

3.2 The list of simulated signal samples used in the analysis. $\mathrm{H}^{0}$ and $\mathrm{X}$ mass values are presented along with three different lifetimes for X. After the boost, the mean transverse decay lengths are of approximately $2 \mathrm{~cm}, 20 \mathrm{~cm}$ and $200 \mathrm{~cm}$ with respect to the laboratory frame. . . . . . . . . . .

3.3 The simulated background samples used in the analysis. The DYJetsToLL samples are the leading background and include Drell-Yan production of all three lepton flavours. The QCD background is smaller. It is modelled with the Mu-Enriched QCD samples. The Mu-Enriched QCD samples contain QCD events where there is at least one generator level muon with $p_{\mathrm{T}}>15 \mathrm{GeV} / c$ (or $5 \mathrm{GeV} / c$ at low $\hat{p_{\mathrm{T}}}$ ). All samples are from the Summer 2012 DR53X production. The event weighting factor is shown for an integrated luminosity of $20 \mathrm{fb}^{-1}$. The cross section includes the efficiency of the generator-level filter, if applicable. . . . . . . . . . . .

4.1 Summary of the analysis selection. . . . . . . . . . . . .

4.2 Dimuon candidate selection efficiencies. Each cut efficiency is the fraction of the candidates passing the cut given that the previous ones are already passed. Preselection is a cut that requires the presence of at least two muons with a transverse momentum, $p_{\mathrm{T}}>26 \mathrm{GeV} / c$ in a triggered event. The cut efficiencies of the signal samples are shown for the events in which there is only one LL particle generated decaying to muons. . . . . . . .

4.3 Efficiencies of the signal MC samples to pass the full selection of the analysis for the events where only one simulated LL particle decays to muons $\left(\epsilon_{1}\right)$, and for the events where two generated LL particles decay to muon pairs $\left(\epsilon_{2}\right) \ldots \ldots \ldots \ldots \ldots \ldots \ldots$

4.4 Efficiencies of the signal MC samples to satisfy the full selection for events within the acceptance. The efficiencies are computed separately for the events where only one simulated LL particle decays to muons $\left(\epsilon_{1}\right)$, and for the events where two generated LL particles decay to muon pairs $\left(\epsilon_{2}\right)$.

6.1 Systematic uncertainties related to the signal selection. The uncertainty specified is a relative uncertainty. The relative uncertainty in the luminosity is $2.6 \% \ldots \ldots \ldots \ldots \ldots \ldots$ 
Table

7.1 Efficiencies of the signal MC samples to satisfy the full selection for the two analyses. The efficiencies for the events in which only one simulated LL particle decays to the muons, $\epsilon_{1}$, and for the ones where there are two generated LL particles decaying to the muon pairs, $\epsilon_{2}$, are shown separately. . . . . . . . . . . . . . . . .

A.1 Reconstruction efficiency of the muon chambers for the muons with several

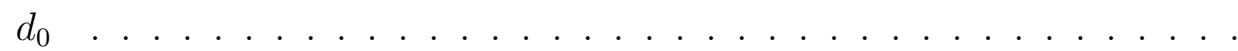




\section{LIST OF FIGURES}

Figure

1.1 (Upper left) Efficiency of the tracker to find a track given a cosmic ray muon as a function of the transverse impact parameter of the muon. Only the tracker muons with $\left|z_{0}\right|<10 \mathrm{~cm}$ are used. (Upper right) Efficiency of the muon chambers for muons with $\left|z_{0}\right|<50 \mathrm{~cm}$. The lower left and lower right plots show the ratio of the efficiency in data to the simulation for the tracker and the muon chambers, respectively. . . . . . . . . .

2.1 The CMS detector in the plane transverse to the beam. . . . . . . . . 5

2.2 One quadrant of the CMS detector in the longitudinal plane. The drift tube (DT) stations are represented by dark green rectangles. The blue and red rectangles denote the four cathode strip chamber (CSC) stations and resistive plate chamber (RPC) stations, respectively. . . . . . . . .

3.1 Transverse view of a simulated event with $M_{\mathrm{H}^{0}}=1000 \mathrm{GeV} / c^{2}$ and $M_{\mathrm{X}}$ $=350 \mathrm{GeV} / c^{2}$. In this event, one $\mathrm{X}$ boson decays to a pair of muons, identified by the hits in the muon system. The other $\mathrm{X}$ boson decays to an electron pair which is not shown in the figure. . . . . . . . . .

4.1 Simple geometry of a dimuon decay. PV and SV denote primary and secondary vertices, respectively. The dimuon momentum vector is represented by the thick red arrow. . . . . . . . . . . . . . . .

4.2 An example event removed by the cosmic rejection cut. Three muons are shown in red, two of which emerge from a cosmic ray muon. A LL candidate is reconstructed from half a cosmic and the other muon in the event that is independent of the cosmic. Note that only the track segments in the muon chambers are representative of the track direction. The segments at smaller radius are instead interpolated to the beamspot position by the visualization software, as it is designed to display prompt tracks.

4.3 The sensitivity of the analysis as a function of generated $L_{x y}$. Although the RSA muon reconstruction efficiency is non-null up to $\approx 5$ meters in the transverse plane, the effective range of the analysis is up to 2.5 meters in the transverse plane due to the trigger efficiency. The light blue line on the plot denotes the generated transverse decay length distribution of the triggered events. The signal sample shown on the diagram has the following mass points: $M_{\mathrm{H}^{0}}=1000 \mathrm{GeV} / c^{2}$ and $M_{\mathrm{X}}=350 \mathrm{GeV} / c^{2}$ with $c \tau=350 \mathrm{~cm} \ldots \ldots \ldots \ldots \ldots \ldots$ 
4.4 Trigger efficiency vs. generated transverse decay length for the $\mathrm{H}^{0} \rightarrow \mathrm{XX}$ signal model with three different mass combinations. The trigger efficiency is computed as the fraction of events within the acceptance that satisfy the trigger requirement. . . . . . . . . . . . . . .

4.5 Distribution of $\Delta R$ separation between the two muons for the dimuon candidates passing the full selection except the one plotted in the control region, $|\Delta \Phi|>\pi / 2$ (left) and in the blinded signal region, $|\Delta \Phi|<\pi / 2$ (right). The dashed lines indicate the cut value of the parameter shown.

4.6 Distribution of the cosine of the 3D opening angle, $\cos (\alpha)$, between the two muons for the dimuon candidates passing the full selection except the one plotted in the control region, $|\Delta \Phi|>\pi / 2$ (left) and blinded signal region, $|\Delta \Phi|<\pi / 2$ (right). . . . . . . . . . . . . . . .

4.7 Distribution of the minimum number of valid muon hits of the two muons for the dimuon candidates passing the full selection except the one plotted in the control region, $|\Delta \Phi|>\pi / 2$ (left) and in the blinded signal region, $|\Delta \Phi|<\pi / 2$ (right). . . . . . . . . . . . . . . . . .

4.8 Distribution of the minimum number of valid muon stations of the two muons for the dimuon candidates passing the full selection except the one plotted in the control region, $|\Delta \Phi|>\pi / 2$ (left) and in the blinded signal region, $|\Delta \Phi|<\pi / 2$ (right) . . . . . . . . . . . . . . . . .

4.9 Distribution of the normalized vertex $\chi^{2}$ of the dimuon candidates passing the full selection except the one plotted in the control region, $|\Delta \Phi|>\pi / 2$ (left) and in the blinded signal region, $|\Delta \Phi|<\pi / 2$ (right). . . . . . . .

4.10 Distribution of the maximum normalized track $\chi^{2}$ of the two muons for the dimuon candidates passing the full selection except the one plotted in the control region, $|\Delta \Phi|>\pi / 2$ (left) and in the blinded signal region, $|\Delta \Phi|<\pi / 2$ (right). . . . . . . . . . . . . . . . . .

4.11 Distribution of the minimum absolute transverse impact parameter significance of the two muons for the dimuon candidates passing the full selection except the one plotted in the control region, $|\Delta \Phi|>\pi / 2$ (left) and in the blinded signal region, $|\Delta \Phi|<\pi / 2$ (right). . . . . . . . . . . . .

4.12 Distribution of the transverse decay length significance of the dimuon candidates passing the full selection except the one plotted in the control region, $|\Delta \Phi|>\pi / 2$ (left) and in the blinded signal region, $|\Delta \Phi|<\pi / 2$ (right). . . . . . . . . . . . . . . . . 
5.1 Collinearity angle, $|\Delta \Phi|$, distribution for the dimuon candidates passing the full selection except $\left|d_{0}\right| / \sigma_{d}, L_{x y} / \sigma_{L_{x y}}$ and $|\Delta \Phi|$ cuts. The signal events plotted have only one LL particle generated decaying to muons. The dashed line indicates the cut value of the parameter shown. . . . . . . .

5.2 Comparisons of $L_{x y} / \sigma_{L_{x y}}$ tail-cumulative distributions between signal , $|\Delta \Phi|<$ $\pi / 2$, and control, $|\Delta \Phi|>\pi / 2$, regions for both data (upper left) and background MC samples (upper right). The full selection is applied with the exception of the $L_{x y} / \sigma_{L_{x y}}$ cut. The $\left|d_{0}\right| / \sigma_{d}$ cut is reversed to $\left|d_{0}\right| / \sigma_{d}<4$. The plots on the bottom left and the bottom right show the statistical significance of the difference between the two regions for data and $\mathrm{MC}$, respectively. The $L_{x y} / \sigma_{L_{x y}}>6$ region is excluded in data to avoid the possible signal contamination. . . . . . . . . . . .

5.3 (Left) $L_{x y} / \sigma_{L_{x y}}$ distribution after applying the full selection except the $L_{x y} / \sigma_{L_{x y}}$ and the tracker track rejection cuts on the data in the control region. The shape is largely determined by the $\left|d_{0}\right| / \sigma_{d}$ cut. The red curve shows the exponential fit for $L_{x y} / \sigma_{L_{x y}}>7$ region. (Right) $L_{x y} / \sigma_{L_{x y}}$ distribution after applying the full selection except the $L_{x y} / \sigma_{L_{x y}}$ cut. Only one event is found with $L_{x y} / \sigma_{L_{x y}}$ larger than $7 \ldots \ldots \ldots$

$5.4 L_{x y} / \sigma_{L_{x y}}$ distribution after applying the full selection except the $L_{x y} / \sigma_{L_{x y}}$ and the tracker track rejection cuts on the data in the control region. The shape is largely determined by the $\left|d_{0}\right| / \sigma_{d}$ cut. The green band shows the variation in the fitted background shape when the exponential fit parameter $\alpha$ is varied by $\pm 20 \%$. . . . . . . . . . . . . . . . .

6.1 Distribution of the number of reconstructed primary vertices for data and the background simulation. The simulation is reweighted according to the procedure explained in the text. The full selection except the tracker track rejection and the cuts on $\left|d_{0}\right| / \sigma_{d}$ and $L_{x y} / \sigma_{L_{x y}}$ is applied. The grey vertical band represents the systematic uncertainty from varying the pileup weights by $\pm 5 \% \ldots \ldots \ldots \ldots \ldots$

6.2 Efficiency of the cosmic muon rejection (left) and of the tracker track rejection (right) vs. the number of reconstructed primary vertices for the signal MC sample with $M_{\mathrm{H}^{0}}=1000 \mathrm{GeV} / c^{2}, M_{\mathrm{X}}=350 \mathrm{GeV} / c^{2}$ and $c \tau=350 \mathrm{~cm}$. All other analysis selection cuts are applied. In both cases no significant dependence on the number of reconstructed primary vertices is observed. 
6.3 Efficiency of the cosmic muon rejection (left) and of the tracker track rejection (right) vs. the number of reconstructed primary vertices for the signal MC sample with $M_{\mathrm{H}^{0}}=1000 \mathrm{GeV} / c^{2}, M_{\mathrm{X}}=350 \mathrm{GeV} / c^{2}$ and $c \tau=350 \mathrm{~cm}$. All other analysis selection cuts are applied except the $\left|d_{0}\right| / \sigma_{d}$ and $L_{x y} / \sigma_{L_{x y}}$ cuts. The minimum muon valid station requirement is also loosened for both plots. In both cases no significant dependence on the number of reconstructed primary vertices is observed. . . . . . . . . . .

6.4 Number of degrees of freedom (left) and timeAtIpInOut (right) in cosmic data and simulation for all muon candidates. . . . . . . . . . . . . .

6.5 timeAtIpInOut vs $\phi$ for data (left) and MC (right) for all muon candidates.

6.6 Number of degrees of freedom (left) and timeAtIpInOut (right) in cosmic data and simulation for muon candidates in the top half of CMS passing the timing selection. . . . . . . . . . . . . .

6.7 timeAtIpInOut vs $\phi$ for data (left) and MC (right) for muon candidates in the top half of CMS passing the timing selection. . . . . . . . . .

6.8 Comparison of kinematic distributions in cosmic data and simulation for all muon candidates. . . . . . . . . . . . . . . . .

6.9 Comparison of kinematic distributions in cosmic data and simulation for muon candidates passing the selection detailed in the text. . . . . . . .

6.10 RSA muon reconstruction and selection efficiency measured by requiring the presence of a reconstructed track in the silicon tracker as a function of $\left|d_{0}\right| \ldots \ldots \ldots \ldots \ldots \ldots \ldots \ldots$

6.11 RSA muon reconstruction and selection efficiency measured using only the muon chambers as a function of $\left|d_{0}\right|$ for $\left|z_{0}\right|<50 \mathrm{~cm} . \ldots \ldots$

$6.12\left|d_{0}\right|$ distributions of both muons from the decay of the same LL particle in Monte Carlo signal samples. From left to right, $M_{X}=20,50,150$, and $350 \mathrm{GeV} / c^{2}$ and from top to bottom, $M_{H}=1000,400,200$, and $125 \mathrm{GeV} / c^{2}$. The generated $c \tau$ of each sample can be found from Figure 6.13. . . . .

6.13 Ratio of the weighted mean efficiencies convoluted with the signal MC distributions. . . . . . . . . . . . . . . . . .

6.14 Trigger efficiency of HLT_L2DoubleMu23_NoVertex vs. $p_{T}$ of the probe muon for both data and $\mathrm{Z} \rightarrow \mu \mu$ simulation, obtained with the Tag and Probe method. . . . . . . . . . . . . . . . . . . . .

6.15 Ratio of HLT_L2DoubleMu23_NoVertex_2Cha_Angle2p5 trigger efficiency to HLT_L2DoubleMu23_NoVertex trigger efficiency vs. $p_{T}$ for both data and $\mathrm{Z} \rightarrow \mu \mu$ simulation. . . . . . . . . . . . . . . . 
6.16 Trigger efficiency of HLT_L2DoubleMu23_NoVertex_2Cha_Angle2p5 vs. generated decay length, $c \tau$, for the signal simulated events with $M_{\mathrm{H}^{0}}=$ $1000 \mathrm{GeV} / c^{2}$ and $M_{\mathrm{X}}=350 \mathrm{GeV} / c^{2} ; M_{\mathrm{H}^{0}}=400 \mathrm{GeV} / c^{2}$ and $M_{\mathrm{X}}=20 \mathrm{GeV} / c^{2}$. Lifetime reweighting is applied to cover the full $c \tau$ range. The trigger efficiency starts to drop when $c \tau>10 \mathrm{~cm}$. . . . . . . . . . . . . . .

7.1 95\% CL upper limits on $\sigma\left(\mathrm{H}^{0} \rightarrow \mathrm{XX}\right) \mathrm{B}\left(\mathrm{X} \rightarrow \ell^{+} \ell^{-}\right)$for $M_{H^{0}}=1000 \mathrm{GeV} / c^{2}$, $400 \mathrm{GeV} / c^{2}, 200 \mathrm{GeV} / c^{2}$ and $125 \mathrm{GeV} / c^{2}$ with various $\mathrm{X}$ mass points. The limits derived for $\mathrm{B} \epsilon_{1} \ll 1$ are illustrated by the solid curves, whereas the dotted curves represent those for $B=1$ (the dotted curves are difficult to discern due to the overlap with the solid curves). Green shaded bands show the $\pm 1 \sigma$ range of variation of the expected $95 \%$ CL limits. . . . .

7.2 Signal efficiency ratio of the two analyses $\epsilon_{R S A} / \epsilon_{t r k}$ for $\mathrm{H}^{0}$ mass values of $1000 \mathrm{GeV} / c^{2}, 400 \mathrm{GeV} / c^{2}, 200 \mathrm{GeV} / c^{2}$ and $125 \mathrm{GeV} / c^{2}$ with various X mass points.

7.3 Combined 95\% CL upper limits on $\sigma\left(\mathrm{H}^{0} \rightarrow \mathrm{XX}\right) \mathrm{B}\left(\mathrm{X} \rightarrow \ell^{+} \ell^{-}\right)$for all $\mathrm{H}^{0}$ mass values of $1000 \mathrm{GeV} / c^{2}, 400 \mathrm{GeV} / c^{2}, 200 \mathrm{GeV} / c^{2}$ and $125 \mathrm{GeV} / c^{2}$ with various $\mathrm{X}$ mass points. The limits derived for $\mathrm{B} \epsilon_{1} \ll 1$ are illustrated by the solid curves, whereas the dotted curves represent those for $\mathrm{B}=1$ (the dotted curves are difficult to discern due to the overlap with the solid curves). Green shaded bands show the $\pm 1 \sigma$ range of variation of the expected $95 \%$ CL limits. . . . . . . . . . . . . . . .

A.1 $p_{T}$ resolution vs. the number of valid hits for the prompt muons . . . .

A.2 $p_{T}$ resolution vs. the number of valid hits for the displaced muons with

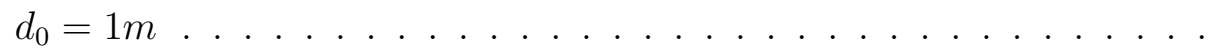

A.3 $d_{0}$ resolution vs. the number of valid hits for the prompt muons . . . 66

A.4 $d_{0}$ resolution vs. the number of valid hits for the displaced muons with $d_{0}=1 m \ldots \ldots \ldots \ldots \ldots \ldots \ldots$. . . . . . . . . . . . . . 67

A.5 $p_{T}$ resolution vs. $p_{T}$ significance for the prompt muons $\ldots \ldots$. . . . 67

A.6 $p_{T}$ resolution vs. $p_{T}$ significance for the displaced muons with $d_{0}=1 \mathrm{~m} \quad 67$

A.7 $d_{0}$ resolution vs. $d_{0}$ significance for the prompt muons . . . . . . . . 68

A.8 $d_{0}$ resolution vs. $d_{0}$ significance for the displaced muons with $d_{0}=1 \mathrm{~m}$. 68

A.9 $d_{0}$ significance vs. the number of valid hits for the prompt muons . . . 68

A.10 $d_{0}$ significance vs. the number of valid hits for the displaced muons with

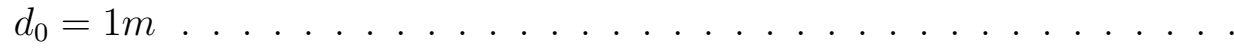

A.11 $p_{T}$ significance vs. the number of valid hits for the prompt muons . . . 
A.12 $p_{T}$ significance vs. the number of valid hits for the displaced muons with

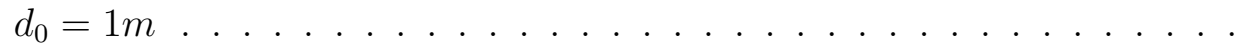

A.13 The difference between reco $d_{0}$ and gen $d_{0}$ vs. $d_{0}$ significance for the prompt muons . . . . . . . . . . . . . . . . . . . .

A.14 The difference between reco $d_{0}$ and gen $d_{0}$ vs. $d_{0}$ significance for the displaced muons with $d_{0}=1 \mathrm{~m} \ldots \ldots \ldots$. . . . . . . . . 70

A.15 Reconstruction efficiency vs. $d_{0}$ for RSA muons with $p_{T}=10 \mathrm{GeV} / c$. . $\quad 72$

A.16 Reconstruction efficiency vs. $d_{0}$ for RSA muons with $p_{T}=100 \mathrm{GeV} / c \ldots$

A.17 Reconstruction efficiency vs. $d_{0}$ for RSA muons with $p_{T}=1000 \mathrm{GeV} / c$.

B.1 (Left) Distribution of the number of valid hits for the in-time muons in the events rejected by the cosmic rejection cut. (Right) Same distribution for the out-of-time muons. There is an obvious shift between the two distributions, suggesting that out-of-time muons, in general, have lower number of hits in the muon chambers. . . . . . . . . . . . . . . . .

B.2 Distribution of the minimum valid muon hits of the two muons for the dimuon candidates passing the full selection except the cut on the minimum valid muon hits. This plot is obtained for the signal sample: $M_{\mathrm{H}^{0}}=$ $1000 \mathrm{GeV} / c^{2}$ and $M_{\mathrm{X}}=350 \mathrm{GeV} / c^{2}$ with $c \tau=350 \mathrm{~cm} \ldots . . . .$.

C.1 Trigger efficiency of HLT_L2DoubleMu23_NoVertex vs. $p_{T}$ of the probe muon for both data and $\mathrm{Z} \rightarrow \mu \mu$ simulation, obtained with the Tag and Probe method. . . . . . . . . . . . . . . . . . . .

C.2 The plot compares HLT_L2DoubleMu23_NoVertex trigger efficiency results from the fitting, already presented in Figure 6.14 and with those from the counting method for the MC sample. . . . . . . . . . . . . . . .

C.3 MC fitting results of tag-probe dimuon mass distributions for each $p_{T}$ bin with Tag and Probe applied for the trigger, HLT_L2DoubleMu23_NoVertex. Note that the range of each $p_{T}$ bin is indicated under each plot. . . . .

C.4 Data fitting results of tag-probe dimuon mass distributions for each $p_{T}$ bin with Tag and Probe applied for the trigger, HLT_L2DoubleMu23_NoVertex. Note that the range of each $p_{T}$ bin is indicated under each plot. . . . .

C.5 HLT_L2DoubleMu23_NoVertex trigger efficiency vs. $p_{T}$ graph. The results from fitting, counting and MC Truth fitting methods are compared. Note that Tag and Probe method is applied. . . . . . . . . . . . . . .

C.6 HLT_L2DoubleMu23_NoVertex trigger efficiency vs. $|\eta|$ graph for both the simulation and data with Tag and Probe applied. . . . . . . . . . . 
C.7 HLT_L2DoubleMu23_NoVertex trigger efficiency distribution on the $p_{T}$ and $|\eta|$ plane for MC with Tag and Probe applied. . . . . . . . . . . . . . .

C.8 HLT_L2DoubleMu23_NoVertex trigger efficiency distribution on the $p_{T}$ and $|\eta|$ plane for data with Tag and Probe applied. . . . . . . . . . . . .

C.9 The ratio of HLT_L2DoubleMu23_NoVertex_2Cha_Angle2p5 trigger efficiency to HLT_L2DoubleMu23_NoVertex trigger efficiency vs. $p_{T}$ graph for both data and the simulation. . . . . . . . . . . . . . . . .

C.10 MC fitting results of dimuon mass distributions. The ratio of the integrals of the two curves in each $p_{T}$ bin gives the fraction of dimuons matched to HLT_L2DoubleMu23_NoVertex_2Cha_Angle2p5 trigger given that HLT_L2DoubleMu23_NoVertex is fired. . . . . . . . . . . . . . . .

C.11 Data fitting results of dimuon mass distributions. The ratio of the integrals of the two curves in each $p_{T}$ bin gives the fraction of dimuons matched to HLT_L2DoubleMu23_NoVertex_2Cha_Angle2p5 trigger given that HLT_L2DoubleMu23_NoVertex is fired. . . . . . . . . . . . . . .

C.12 The ratio of HLT_L2DoubleMu23_NoVertex_2Cha_Angle2p5 trigger efficiency to HLT_L2DoubleMu23_NoVertex trigger efficiency vs. $\cos \theta$. Note that $\theta$ is the $3 \mathrm{D}$ angle between the two muons. . . . . . . . . .

D.1 (Left) Distribution of the minimum absolute transverse impact parameter significance of the two muons for the dimuon candidates passing the selection. (Right) Distribution of the transverse decay length significance of the dimuon candidates passing the selection. The selection here is defined as the full analysis selection except $\left|d_{0}\right| / \sigma_{d}, L_{x y} / \sigma_{L_{x y}}$ and track rejection cuts in the control region. In addition to the selection defined, no cut is applied to the parameter shown in the plots. . . . . . . . . .

D.2 (Left) Distribution of the cosine of the 3D opening angle, $\cos (\alpha)$, between the two muons for the dimuon candidates passing the selection. (Right) Distribution of $\Delta R$ separation between the two muons for the dimuon candidates passing the selection. . . . . . . . . . . .

D.3 (Left) Distribution of the maximum normalized track $\chi^{2}$ of the two muons for the dimuon candidates passing the selection. (Right) Distribution of the normalized vertex $\chi^{2}$ of the dimuon candidates passing the selection.

D.4 (Left) Distribution of the minimum number of valid muon hits of the two muons for the dimuon candidates passing the selection. (Right) Distribution of the minimum number of valid muon stations of the two muons for the dimuon candidates passing the selection. . . . . . . . . .

D.5 Distribution of the mass for the dimuon candidates passing the selection. 
E.1 Dimuon mass distribution for both background MC, shown in red, and data, in blue. Note that the number of events is normalized. . . . . . .

E.2 Dimuon mass distribution for background MC (left) and data (right) fitted by Crystal Ball function. Note that the number of events is normalized.

E.3 Dimuon mass distribution for both rescaled background MC, shown in red, and data, in blue. $p_{\mathrm{T}}$ is increased by a factor of $1.13 \%$ for each simulated muon. The dimuon masses are recomputed. Note that the number of events is normalized. . . . . . . . . . . . . . . .

E.4 Dimuon mass distribution for both rescaled background MC, shown in red, and data, in blue. $p_{\mathrm{T}}$ is increased by a factor of $10 \%$ for each simulated muon. The dimuon masses are recomputed. Note that the number of events is normalized. . . . . . . . . . . . . . . . . .

F.1 Distribution of the distance between primary and secondary vertices in $\mathrm{x}$ and $\mathrm{y}$ planes for the simulated $\mathrm{Z} \rightarrow \mu^{+} \mu^{-}$events. The shapes look gaussian indicating the symmetry of the decay. . . . . . . . . . . .

F.2 Distribution of the transverse decay length for the simulated $\mathrm{Z} \rightarrow \mu^{+} \mu^{-}$ events. Since it is square root of the square sum of the two gaussian distributions, the resolution of $L_{x y}$ can not be trivially extracted from this shape. . . . . . . . . . . . . . . . . .

F.3 Distribution of the distance between primary and secondary vertices in $\mathrm{x}$ and y planes for the simulated $\mathrm{Z} \rightarrow \mu^{+} \mu^{-}$events. Bold red curve is the gaussian fit applied to one of the two. The sigma value of the fit function

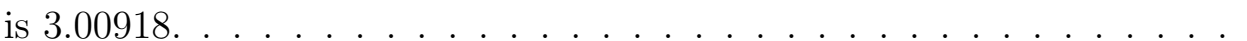

G.1 Correlation of the reconstructed Lxy (left), $L_{x y}$ error (center), and $L_{x y}$ significance (right) with the generated $L_{x y}$. The $L_{x y}$ error defaults to a value around $100 \mathrm{~cm}$ after approximately 3.4 meters. This is outside the effective region of the analysis that is limited to approximately 2.5 meters by the trigger efficiency. . . . . . . . . . . . . . . . .

G.2 Distribution of the generated $L_{x y}$ for all generated long-lived particles decaying to muons (blue), for those that have both muons reconstructed (red), and for the ones where a secondary vertex is also successfully reconstructed (green). . . . . . . . . . . . . . . . . . . . . . . . . . . . . . .

G.3 Efficiency to reconstruct a secondary vertex in signal MC events for $M_{H^{0}}=$ $400 \mathrm{GeV} / c^{2}, M_{X}=50 \mathrm{GeV} / c^{2}$ and an average lifetime of $800 \mathrm{~cm}$. . . 
G.4 Distribution of the number of cosmic events in data (left) and simulation (right) with two reconstructed RSA muons within the acceptance (black) and with the additional requirement of a reconstructed secondary vertex (red) as a function of the $d_{0}$ of the muon with the highest $p_{T} . \ldots$

G.5 Efficiency to reconstruct a (fake) secondary vertex in cosmic data (black) and simulation (red) as a function of the $d_{0}$ of the highest $p_{T}$ RSA muon in the event. . . . . . . . . . . . . . . . . . .

H.1 (Left) $L_{x y} / \sigma_{L_{x y}}$ cumulative distribution as all the remaining cuts are applied. (Right) $\left|d_{0}\right| / \sigma_{d}$ cumulative distribution. The most conservative estimate of background comes from the left one, $0.23 \pm 0.24$, suggesting that the systematic uncertainty on the number of estimated background can be set as high as 0.47 . Note that the red curves represent the exponential unbinned fit functions. . . . . . . . . . . . . . . . . . 


\begin{abstract}
Solmaz, Melih M.S., Purdue University, August 2014. Search for New Physical Phenomena via Displaced Muon Signatures with the CMS Detector at the LHC. Major Professor: Ian P. Shipsey.

The first search at the LHC for long-lived neutral particles decaying to pairs of muons by using only the muon chambers is presented. Events were collected by the CMS detector during pp collisions at $\sqrt{s}=8 \mathrm{TeV}$ and selected from data samples corresponding to $20.5 \mathrm{fb}^{-1}$ of integrated luminosity. No background events are expected after the full analysis selection. Expected upper limits are derived for a model which predicts a heavy scalar decaying to two long-lived particles, each of which can decay to muon pairs. Combined expected upper limits with an analysis utilizing the CMS silicon tracker to search for the same signature are also presented.
\end{abstract}




\section{CHAPTER 1. INTRODUCTION}

Massive long-lived particles conjectured by several new physics models, such as "split SUSY" [1] or SUSY with very weak R-parity violation [2], "hidden valley" models [3] and Z' models that contain long-lived neutrinos [4], might be produced at the LHC. In the models where the long-lived massive particles decay to lepton pairs, they can be differentiated from Standard Model (SM) particles by virtue of the significant distance they travel in the volume of the detector.

As a benchmark for this physics signature, a particular model is considered to quantify the sensitivity of the analysis. This model postulates pair production of long-lived $\mathrm{X}$ particles by the decay of a non-SM Higgs boson, $\mathrm{H}^{0} \rightarrow \mathrm{XX}$, where $\mathrm{H}^{0}$ is produced by gluon-gluon fusion and $\mathrm{X}$ is a spinless boson decaying to lepton pairs, $\mathrm{X} \rightarrow \ell^{+} \ell^{-}[5]$.

This study presents the blinded results of a search for long-lived neutral particles decaying to muon pairs reconstructed using only the muon chambers of the Compact Muon Solenoid (CMS) detector. The analysis uses data taken during 2012 in pp collision at $\sqrt{s}=8 \mathrm{TeV}$, corresponding to a total integrated luminosity of $20.5 \mathrm{fb}^{-1}$. It shares some similarities with another CMS analysis [6], searching for particles of the same nature yielding displaced electron and muon signals by utilizing both the silicon tracker and the muon system for particle reconstruction. Nevertheless, the two analyses are orthogonal by construction, as explained later in this document.

Although the tracker-based analysis benefits from the precision with which tracks are measured in the silicon tracker, the major constraint comes from the fact that the reconstruction efficiency for a track in the silicon tracker is essentially zero for tracks with transverse impact parameter $\left(d_{0}\right)$, the closest distance between the track and the interaction point in the transverse plane of the detector, greater than $40 \mathrm{~cm}$. The tracker-based analysis has little sensitivity to particles with longer lifetimes. On 
the other hand, the muon chambers give non-vanishing reconstruction efficiency even a few meters away from the interaction point. To illustrate this, the reconstruction efficiencies of the tracker and the muon chambers as a function of $d_{0}$ are given in Figure 1.1. Additional selection requirements are applied to derive the reconstruction efficiency of the muon system, including quality selection efficiency. More details can be found in Chapter 6.3. Therefore, the muon chambers can be used to extend the lifetime sensitivity of this analysis. Note, however, that the muon chambers have a much lower muon $p_{\mathrm{T}}$ resolution and higher level of cosmic muon background compared to the tracker-based analysis. Importantly, the double muon trigger that is used to collect the events has a vanishing efficiency beyond 2.5 meters of the collision point in the transverse plane. Hence, the effective range of the analysis is 2.5 meters, which is only halfway through the muon chambers.

The analysis is fully complementary to the tracker-based analysis in that the displaced muons reconstructed by the muon chambers that are matched to the tracker muons are rejected. That is, the set of events passing the full selection of the analysis does not overlap with the one satisfying the selection criteria of the tracker-based analysis.

The D0 Collaboration has performed similar searches for leptons from displaced decays within its tracker volume $[7,8]$, yet the scope of these searches covers a much smaller kinematic phase space region than CMS. The ATLAS Collaboration has performed searches that are sensitive to decay lengths up to about $20 \mathrm{~m}$ by exploiting the ATLAS muon spectrometer $[9,10]$, using different decay channels from those considered in this analysis. 

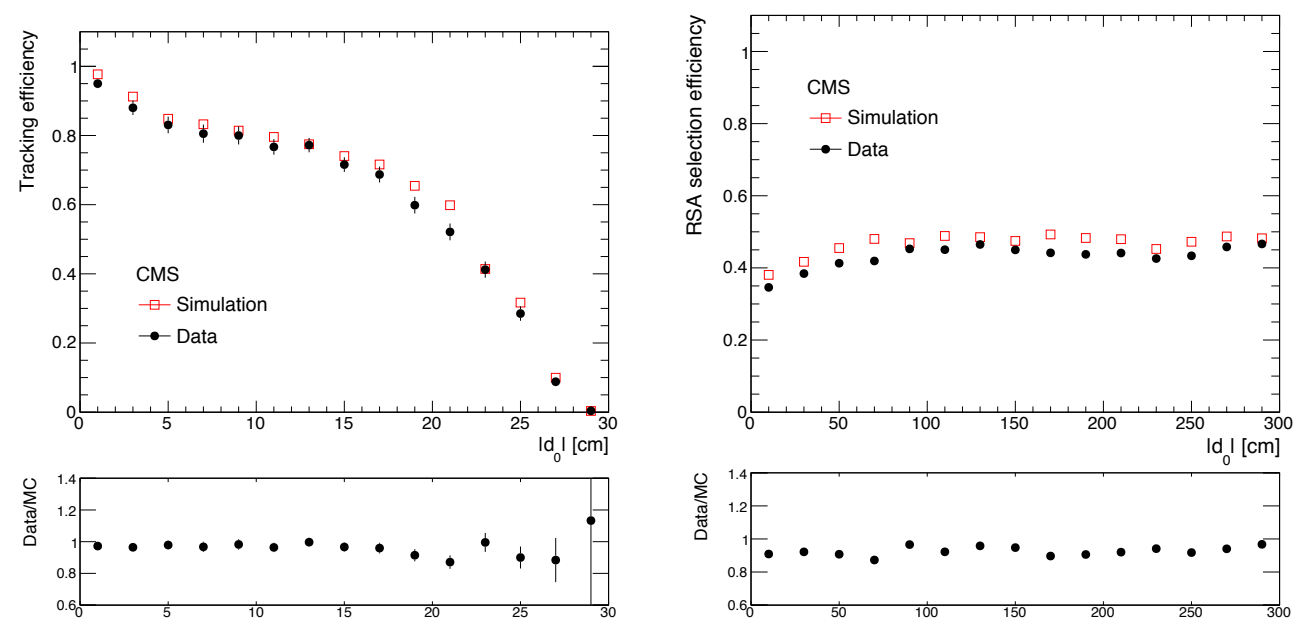

Figure 1.1.: (Upper left) Efficiency of the tracker to find a track given a cosmic ray muon as a function of the transverse impact parameter of the muon. Only the tracker muons with $\left|z_{0}\right|<10 \mathrm{~cm}$ are used. (Upper right) Efficiency of the muon chambers for muons with $\left|z_{0}\right|<50 \mathrm{~cm}$. The lower left and lower right plots show the ratio of the efficiency in data to the simulation for the tracker and the muon chambers, respectively. 


\section{CHAPTER 2. THE CMS DETECTOR}

The central feature of the CMS apparatus [11] is a superconducting solenoid of $6 \mathrm{~m}$ internal diameter providing an axial field of $3.8 \mathrm{~T}$. Within the field volume are the silicon pixel, strip tracker, the lead-tungstate crystal electromagnetic calorimeter (ECAL) and the brass/scintillator hadron calorimeter (HCAL). Muons are identified in gas-ionisation detectors embedded in the steel magnetic-flux return yoke of the solenoid. The transverse view of the detector is illustrated in Figure 2.1.

The silicon tracker can reconstruct the trajectories of charged particles such as muons, electrons and hadrons as well as their momentum with high precision. It is composed of pixel detectors (three barrel layers and two forward disks on either end of the detector) surrounded by strip detectors (ten barrel layers plus three inner disks and nine forward disks at each end of the detector). The tracker covers the pseudorapidity range $|\eta|<2.5$, where $\eta=-\ln [\tan (\theta / 2)]$ and $\theta$ is the polar angle with respect to the anticlockwise-beam direction.

In order to stop electrons and photons, the electromagnetic calorimeter is placed on the periphery of the silicon tracker. The ECAL consists of nearly 76000 lead tungstate crystals in a barrel and two endcap sections, which provide coverage in pseudorapidity $|\eta|<3$. The hadron calorimeter is between the muon chambers and the electromagnetic calorimeter. It measures the energy of hadrons and it is made up of barrel, endcap and forward sections.

Muons are measured in the pseudorapidity range $|\eta|<2.4$ with detection planes based on one of three technologies: drift tubes in the barrel region, cathode strip chambers in the endcaps, and resistive plate chambers in the barrel and endcaps. The muon system, shown in Figure 2.2 has three main functions: triggering on muons, muon identification, and the improvement of muon momentum measurement. The drift tube subsystem, which covers $|\eta|<1.2$ region, is responsible for determining 


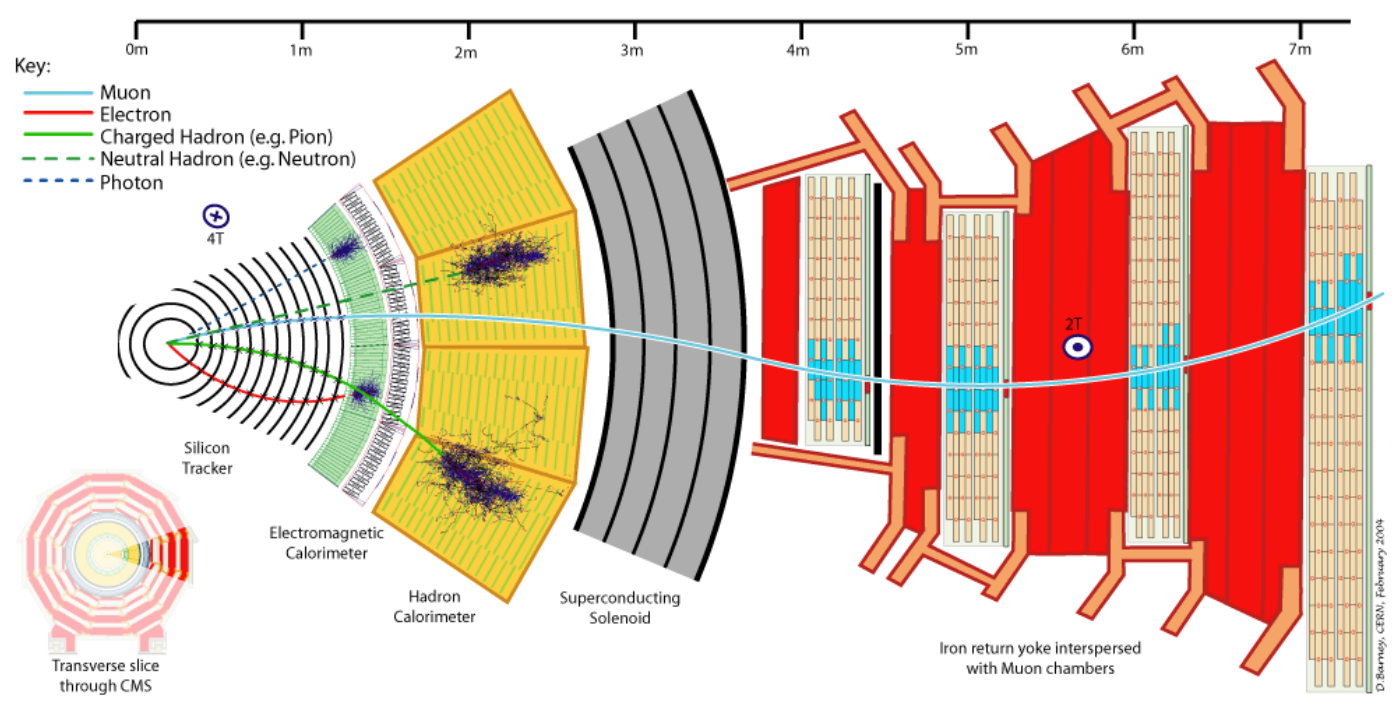

Figure 2.1.: The CMS detector in the plane transverse to the beam. 
the muon position through the process of ionization in the gas tubes. Cathode strip chambers consist of anode wires and cathode strips positioned perpendicular to each other. They provide two position coordinates in the region, $0.9<|\eta|<2.4$. The resistive plate chambers located in the pseudorapidity range of $|\eta|<1.6$ provide additional fast muon trigger capability. Track reconstruction can be achieved in the tracker and the muon system independently and it can be improved by combining the two. Muon reconstruction performance has been studied in great detail with data $[12]$.

The first level of the CMS trigger system, composed of custom hardware processors, selects events of interest using information from the calorimeters and the muon detectors. A high-level trigger processor farm then employs the full event information to further decrease the event rate. 


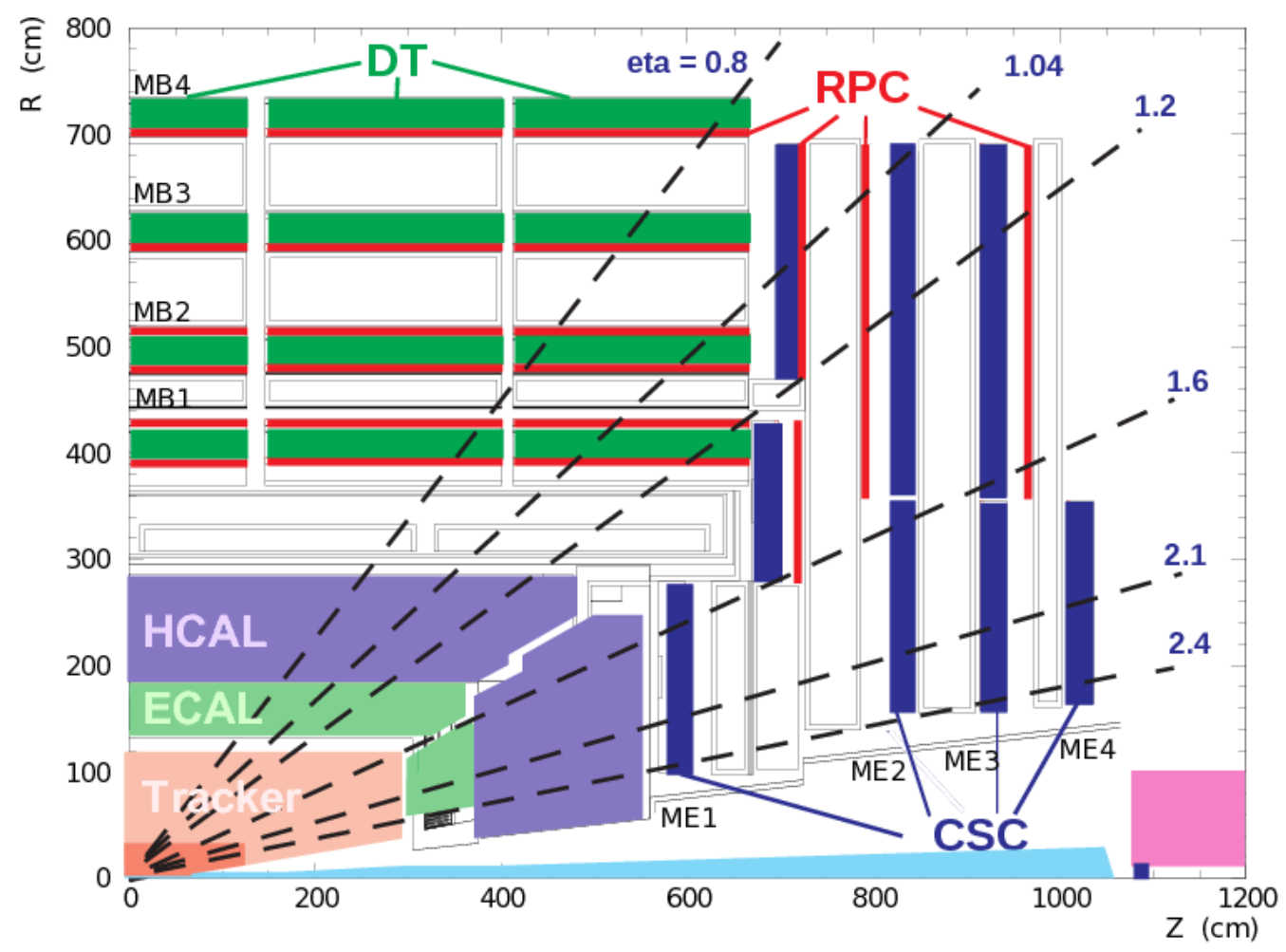

Figure 2.2.: One quadrant of the CMS detector in the longitudinal plane. The drift tube (DT) stations are represented by dark green rectangles. The blue and red rectangles denote the four cathode strip chamber (CSC) stations and resistive plate chamber (RPC) stations, respectively. 


\section{CHAPTER 3. DATA AND MONTE CARLO SIMULATION SAMPLES}

The analysis uses data taken from pp collisions at a centre-of-mass energy of 8 TeV corresponding to an integrated luminosity of $20.5 \pm 0.5 \mathrm{fb}^{-1}$. The CMS datasets utilized are reprocessed under CMSSW 5_3_7 in January 2013. They are known as the "rereco" data. Table 3.1 lists them along with the associated good run range.

Table 3.1.: The CMS datasets used by the analysis.

\begin{tabular}{|c|c|}
\hline Dataset & Run range \\
\hline Run2012A-22Jan2013-v1/AOD & $190456-193621$ \\
Run2012B-22Jan2013-v1/AOD & $193833-196531$ \\
Run2012C-22Jan2013-v1/AOD & $198022-203742$ \\
Run2012D-22Jan2013-v1/AOD & $203777-208686$ \\
\hline
\end{tabular}

HLT_L2DoubleMu23_NoVertex_2Cha_Angle2p5 is the Level 2 (L2) double muon trigger that collects the events used in this analysis. It requires two muons in an event, each reconstructed in the muon detectors without imposing any beam spot constraint and having $p_{\mathrm{T}}>23 \mathrm{GeV} / c$. Both muons are also required to have at least two reconstructed hits in at least two cathode strip chambers (CSC) or drift tubes (DT). To prevent cosmic ray muons from passing these criteria, the opening angle between the two muons must be less than 2.5 radians. The trigger is independent of the silicon tracker activity.

The simulated signal samples are generated using PYTHIA V6.426 [13] to simulate $\mathrm{H}^{0}$ production through gluon fusion $\left(g g \rightarrow \mathrm{H}^{0}\right)$. Subsequently the $\mathrm{H}^{0}$ is forced to decay to $\mathrm{XX}$, with the $\mathrm{X}$ bosons each decaying to lepton pairs $\left(\mathrm{X} \rightarrow \ell^{+} \ell^{-}\right)$. The analysis focuses on the final states with at least one muon pair. The generated 
samples tabulated in Table 3.2 have $M_{\mathrm{H}^{0}}=125,200,400,1000 \mathrm{GeV} / c^{2}$ and $M_{\mathrm{X}}=20$, $50,150,350 \mathrm{GeV} / c^{2}$. Each sample is produced with three $\mathrm{X}$ boson lifetimes. After the boost, mean transverse decay lengths are of approximately $2 \mathrm{~cm}, 20 \mathrm{~cm}$ and $200 \mathrm{~cm}$ with respect to the laboratory frame. The sensitivity of the analysis is determined only for the decays with the longest lifetime in each sample. Figure 3.1 displays a simulated event with $M_{\mathrm{H}^{0}}=1000 \mathrm{GeV} / c^{2}$ and $M_{\mathrm{X}}=350 \mathrm{GeV} / c^{2}$.

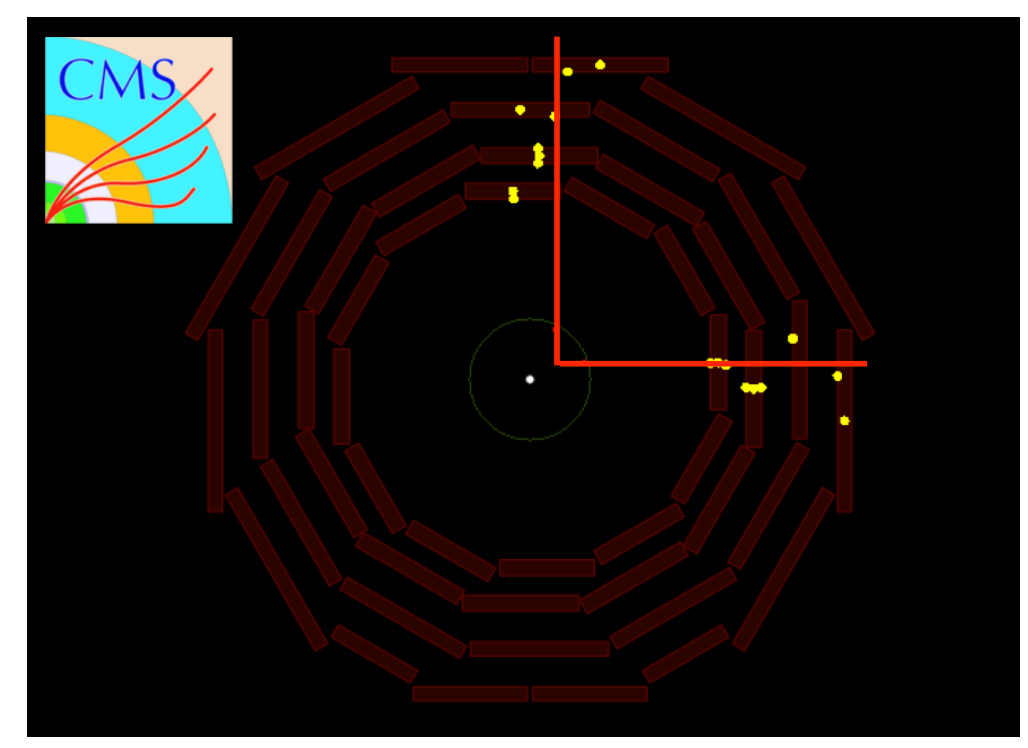

Figure 3.1.: Transverse view of a simulated event with $M_{\mathrm{H}^{0}}=1000 \mathrm{GeV} / c^{2}$ and $M_{\mathrm{X}}$ $=350 \mathrm{GeV} / \mathrm{c}^{2}$. In this event, one $\mathrm{X}$ boson decays to a pair of muons, identified by the hits in the muon system. The other $\mathrm{X}$ boson decays to an electron pair which is not shown in the figure.

All MC background samples, reconstructed under CMSSW 5_3_2, used in the analysis are listed in Table 3.3. They are generated with PYTHIA and correspond to 'Summer12 DR53X' production. The major background for this analysis comes from the Drell-Yan process yielding dileptons, $\mu^{+} \mu^{-}$and $\tau^{+} \tau^{-}$at significant rates. Even 
though the branching ratio is low, tau decays might also lead to displaced muons that can fake the signals we are looking for. The Drell-Yan background is simulated at Next-Leading-Order (NLO) with POWHEG [14]. Other simulated backgrounds are $t \bar{t}, \mathrm{~W} / \mathrm{Z}$ boson pair production with leptonic decays, and QCD multijet events. All these backgrounds produce negligible contributions. However, the random cosmic background is not simulated and the background MC cannot provide a good description of the expected background. The expected background is estimated from the data. In all the samples, the response of the detector is simulated in detail using GEANT4 [15]. The samples are then processed through the trigger emulation and event reconstruction chain of the CMS experiment.

Table 3.2.: The list of simulated signal samples used in the analysis. $\mathrm{H}^{0}$ and $\mathrm{X}$ mass values are presented along with three different lifetimes for X. After the boost, the mean transverse decay lengths are of approximately $2 \mathrm{~cm}, 20 \mathrm{~cm}$ and $200 \mathrm{~cm}$ with respect to the laboratory frame.

\begin{tabular}{|c|c|c|}
\hline$M_{\mathrm{H}^{0}}\left(\mathrm{GeV} / c^{2}\right)$ & $M_{\mathrm{X}}\left(\mathrm{GeV} / c^{2}\right)$ & $c \tau(\mathrm{cm})$ \\
\hline 1000 & 350 & $(3.5,35.0,350.0)$ \\
1000 & 150 & $(1.0,10.0,100.0)$ \\
1000 & 50 & $(0.4,4.0,40.0)$ \\
1000 & 20 & $(0.15,1.5,15.0)$ \\
400 & 150 & $(4.0,40.0,400.0)$ \\
400 & 50 & $(0.8,8.0,80.0)$ \\
400 & 20 & $(0.4,4.0,40.0)$ \\
200 & 50 & $(2.0,20.0,200.0)$ \\
200 & 20 & $(0.7,7.0,70.0)$ \\
125 & 50 & $(5.0,50.0,500.0)$ \\
125 & 20 & $(1.3,13.0,130.0)$ \\
\hline
\end{tabular}


Table 3.3.: The simulated background samples used in the analysis. The DYJetsToLL samples are the leading background and include Drell-Yan production of all three lepton flavours. The QCD background is smaller. It is modelled with the Mu-Enriched QCD samples. The Mu-Enriched QCD samples contain QCD events where there is at least one generator level muon with $p_{\mathrm{T}}>15 \mathrm{GeV} / c$ (or $5 \mathrm{GeV} / c$ at low $\hat{p_{\mathrm{T}}}$ ). All samples are from the Summer 2012 DR53X production. The event weighting factor is shown for an integrated luminosity of $20 \mathrm{fb}^{-1}$. The cross section includes the efficiency of the generator-level filter, if applicable.

\begin{tabular}{|l|c|c|c|}
\hline \multicolumn{1}{|c|}{ Dataset name } & $\begin{array}{c}\text { Cross section } \\
(\mathrm{pb})\end{array}$ & Number of events & Weight Factor \\
\hline DYJetsToLL_M-10To50 & $1.25 \mathrm{e}+04$ & $3.78 \mathrm{e}+07$ & 6 \\
DYJetsToLL_M-50 & $3.5 \mathrm{e}+03$ & $3.05 \mathrm{e}+07$ & 2.3 \\
WW & 54.8 & $1 \mathrm{e}+07$ & 0.11 \\
WZ & 33.2 & $1 \mathrm{e}+07$ & 0.0664 \\
ZZ & 17.6 & $9.8 \mathrm{e}+06$ & 0.0359 \\
TTJets_FullLeptMGDecay & 24.8 & $1.21 \mathrm{e}+07$ & 0.041 \\
WJetsToLNu & $3.63 \mathrm{e}+04$ & $1.84 \mathrm{e}+07$ & 39.4 \\
QCD_Pt-15to20_MuEnrichedPt5 & $2.74 \mathrm{e}+06$ & $1.72 \mathrm{e}+06$ & $3.18 \mathrm{e}+04$ \\
QCD_Pt_20_MuEnrichedPt_15 & $1.35 \mathrm{e}+05$ & $2.15 \mathrm{e}+07$ & 125 \\
\hline
\end{tabular}




\section{CHAPTER 4. EVENT RECONSTRUCTION AND SELECTION}

\subsection{Displaced track reconstruction}

By design, the analysis does not use the silicon tracker information in muon track reconstruction. At CMS, there are a handful of algorithms which utilize only the hits in the muon chambers to perform track reconstruction of muons. The two muon collections which are based only on the muon chambers are refittedStandAlone (RSA) and standAlone (SA) muons. Whilst there are structural similarities between the two, the differentiation arises due to the fact that the RSA muon algorithm computes an additional final fit of the tracks by excluding the beam spot, which provides more accuracy for displaced muon measurements. On the other hand, SA muons preserve the inherent bias towards the collision point, which is designed to analyze muons coming directly from the beam spot, so called prompt muons.

The RSA muon collection improves transverse impact parameter, $d_{0}$, and transverse momentum, $p_{T}$, resolutions for displaced muons compared to those of the SA muons, as expected. This has been confirmed in a study reported in Appendix A where the performances of RSA and SA muons are compared. Therefore, the RSA muon collection is chosen for this analysis.

\subsection{Muon selection}

We require the RSA muons to satisfy $p_{\mathrm{T}}>26 \mathrm{GeV} / c$ and pseudorapidity, $|\eta|<2$. The momentum threshold is slightly higher than the corresponding trigger requirement, which is $p_{\mathrm{T}}>23 \mathrm{GeV} / c$, to ensure that the trigger has a good efficiency and its systematic uncertainty is minimal.

A distinctive track rejection step is applied to make the analysis fully complementary to the tracker-based analysis described in Ref. [6] and to exclude prompt 
muons in the most effective way. All muons reconstructed in the muon chambers are rejected if they can be matched to a track reconstructed in the silicon tracker with $p_{T}>10 \mathrm{GeV} / c$. The matching is done by extrapolating the track from the silicon tracker to the muon's innermost hit in the muon system. The track and the muon are considered matched if $\Delta R$ (where $\Delta R=\sqrt{\Delta \phi^{2}+\Delta \eta^{2}}$ between the innermost hit of the muon and the extrapolated position of the tracker track in the muon chambers) is less than 0.1 . The $p_{T}$ requirement on the tracker tracks is relaxed compared to Ref. [6] to account for the low $p_{T}$ resolution of RSA muons. Loosening this requirement further does not lead to the removal of extra prompt events in data.

To select muons of good quality, the fit of the hits in the muon chambers to build each muon track should meet the condition $\chi^{2} /$ dof $<2$. Each muon must have at least 3 muon stations with at least a valid hit. Given non-negligible cosmic muon contamination, each muon is also required to have at least 17 valid hits as a sanity check since in most cases out-of-time muons with cosmic origin tend to have lower number of valid hits compared to in-time muons, that is, muons arising from pp collisions. A detailed study of in-time and out-of-time muons is presented in Appendix B. Finally, muons should have a transverse impact parameter significance, $\left|d_{0}\right| / \sigma_{d}>4$, where $\left|d_{0}\right| / \sigma_{d}$ is the ratio of the transverse impact parameter to its error.

\subsection{Selection of long-lived exotica}

The long-lived (LL) particle candidates are formed by pairing all muons in the event in all possible combinations. There is no opposite charge requirement enforced when building the dimuon candidates to eliminate the unfavourable effect of charge mis-measurement by the muon system. Among all LL candidates that share the same lepton, the one with the smallest $\chi^{2} /$ dof of the secondary vertex, which two muon tracks are fitted to, is kept. The procedure avoids the double counting of muons.

We discard dimuons consistent with coming from $\mathrm{J} / \psi$ and $\Upsilon$ decays and $\gamma$ conversions by requiring an invariant mass greater than $15 \mathrm{GeV} / c^{2}$. Although the tracker 
track rejection step should already remove this background, the minimum mass cut is kept as a sanity check. The two muon tracks are required to form a secondary vertex with $\chi^{2} /$ dof $<4$. The angular difference in the azimuthal plane, $\Delta \Phi$, between the dimuon momentum vector and the vector from the primary vertex to the dilepton vertex should satisfy $|\Delta \Phi|<\pi / 2$, where $\Delta \Phi$ is measured in the range $-\pi<\Delta \Phi<\pi$. The diagram showing the simple geometry of a dimuon decay in Figure 4.1 describes this collinearity angle pictorially. The region, $|\Delta \Phi|<\pi / 2$, is called signal region and the one with $|\Delta \Phi|>\pi / 2$ is defined as control region. The control region should be signal-free, whereas the background should be symmetrically distributed in both regions.

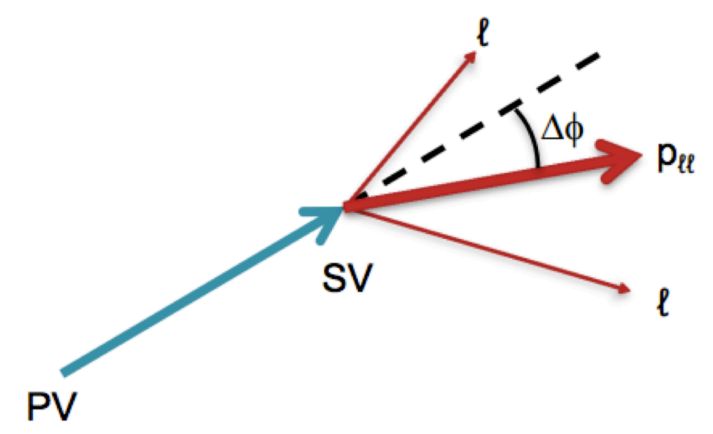

Figure 4.1.: Simple geometry of a dimuon decay. PV and SV denote primary and secondary vertices, respectively. The dimuon momentum vector is represented by the thick red arrow. 
A significant amount of background arises from cosmic rays, which may be reconstructed as back-to-back muons that are often displaced from the primary vertex. Such events should, in principle, be removed at trigger level. However, the trigger requirement $\cos (\alpha)>-0.8$ is tightened to $\cos (\alpha)>-0.75$, where $\alpha$ is the 3D opening angle between the two muons. Furthermore, a dimuon candidate can also be reconstructed from half a cosmic and another (fake or real) muon in the event. To remove these combinations, candidates are rejected when one of the two muons is back-to-back $(\cos (\alpha) \leq-0.75)$ to another muon in the same event that is not included in another dimuon candidate. An example event removed by this cut is shown in Figure 4.2.

The double muon trigger efficiency becomes difficult to model when the two muons are very close to each other. Hence, it is required that the two muons are separated by $\Delta R>0.2$. Finally, LL candidates should have a transverse decay length significance of $L_{x y} / \sigma_{L_{x y}}>12$, where $L_{x y}$ is defined as the distance between the primary and the secondary vertices in the transverse plane and its resolution is studied comprehensively in Appendix F. The full selection is summarized in Table 4.1.

We generate the signal to be within the CMS detector acceptance given by:

- The generated transverse decay length $L_{x y}$ of the LL particle must be $<500 \mathrm{~cm}$.

- The generated muon pseudorapidity must be $|\eta|<2$.

- The generated muon momentum must satisfy $p_{\mathrm{T}}>26 \mathrm{GeV} / c$.

Figure 4.3 demonstrates that our analysis is mostly sensitive to LL particles with long lifetimes while being completely insensitive to prompt events. That plot also suggests that although RSA muons have non-null reconstruction efficiency up to 5 meters away from the beam spot in the transverse plane, as shown in Appendix A, the effective range of the analysis is restricted to 2.5 meters since the dimuon trigger efficiency vanishes around that distance. To illustrate this limitation more clearly, the trigger efficiency given that the event is within the acceptance vs. generated 


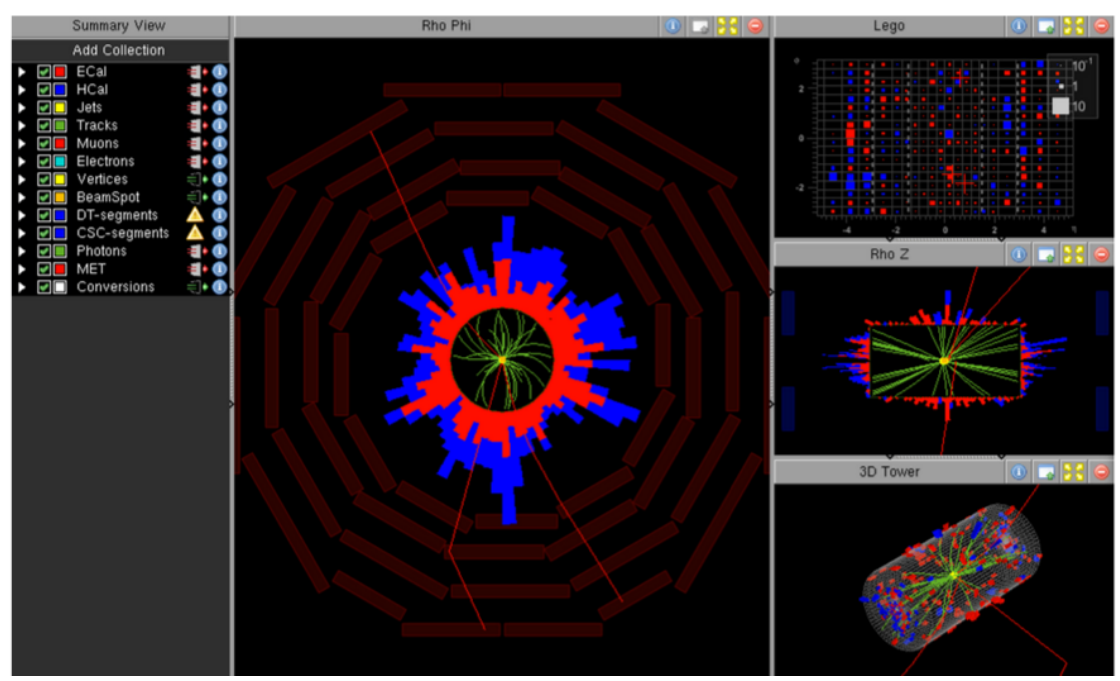

Figure 4.2.: An example event removed by the cosmic rejection cut. Three muons are shown in red, two of which emerge from a cosmic ray muon. A LL candidate is reconstructed from half a cosmic and the other muon in the event that is independent of the cosmic. Note that only the track segments in the muon chambers are representative of the track direction. The segments at smaller radius are instead interpolated to the beamspot position by the visualization software, as it is designed to display prompt tracks. 
transverse decay length, $L_{x y}$, graph is drawn for three different signal MC samples in Figure 4.4.

Table 4.1.: Summary of the analysis selection.

\begin{tabular}{|l||c|}
\hline Selection cut & Cut value \\
\hline Min. track $p_{\mathrm{T}}(\mathrm{GeV} / c)$ & 26 \\
Max. track $|\eta|$ & 2 \\
Max. normalized track $\chi^{2}$ & 2 \\
Max. normalized vertex $\chi^{2}$ & 4 \\
Min. $\Delta R$ between the two muons & 0.2 \\
Min. $\cos (\alpha)$ & -0.75 \\
Min. dimuon mass $\left(\mathrm{GeV} / c^{2}\right)$ & 15 \\
Max. $|\Delta \Phi|$ & $\pi / 2$ \\
Min. number of muon DT $+\mathrm{CSC}$ stations & 3 \\
Min. number of valid muon hits & 17 \\
Min. muon $\left|d_{0}\right| / \sigma_{d}$ & 4 \\
Min. dimuon $L_{x y} / \sigma_{L_{x y}}$ & 12 \\
\hline
\end{tabular}

\subsection{Results of blind analysis}

The blind analysis has been completed with the full selection described above. The plots from Figure 4.5 to Figure 4.12 show the distributions of the cut parameters in both control, $|\Delta \Phi|>\pi / 2$, and signal, $|\Delta \Phi|<\pi / 2$, regions with all the selection applied except the one plotted. No event in data passes the full selection in the control region of the analysis. This implies that the number of expected background events in the signal region of data is zero as well, given the established symmetry between the two regions, as validated in Chapter 5.1. The systematic uncertainty from the expected background is estimated in Chapter 5.2. 


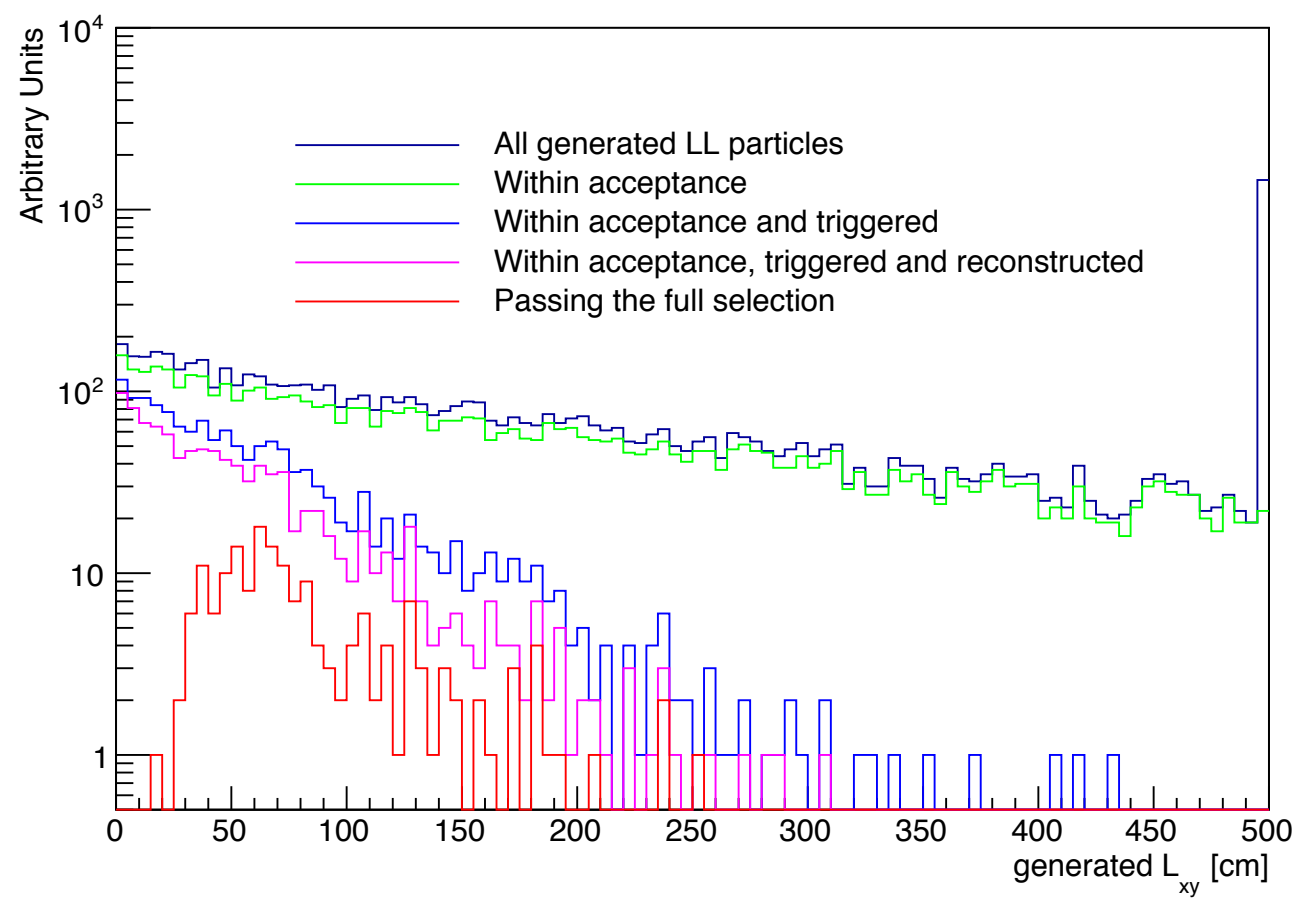

Figure 4.3.: The sensitivity of the analysis as a function of generated $L_{x y}$. Although the RSA muon reconstruction efficiency is non-null up to $\approx 5$ meters in the transverse plane, the effective range of the analysis is up to 2.5 meters in the transverse plane due to the trigger efficiency. The light blue line on the plot denotes the generated transverse decay length distribution of the triggered events. The signal sample shown on the diagram has the following mass points: $M_{\mathrm{H}^{0}}=1000 \mathrm{GeV} / c^{2}$ and $M_{\mathrm{X}}=350 \mathrm{GeV} / c^{2}$ with $c \tau=350 \mathrm{~cm}$. 


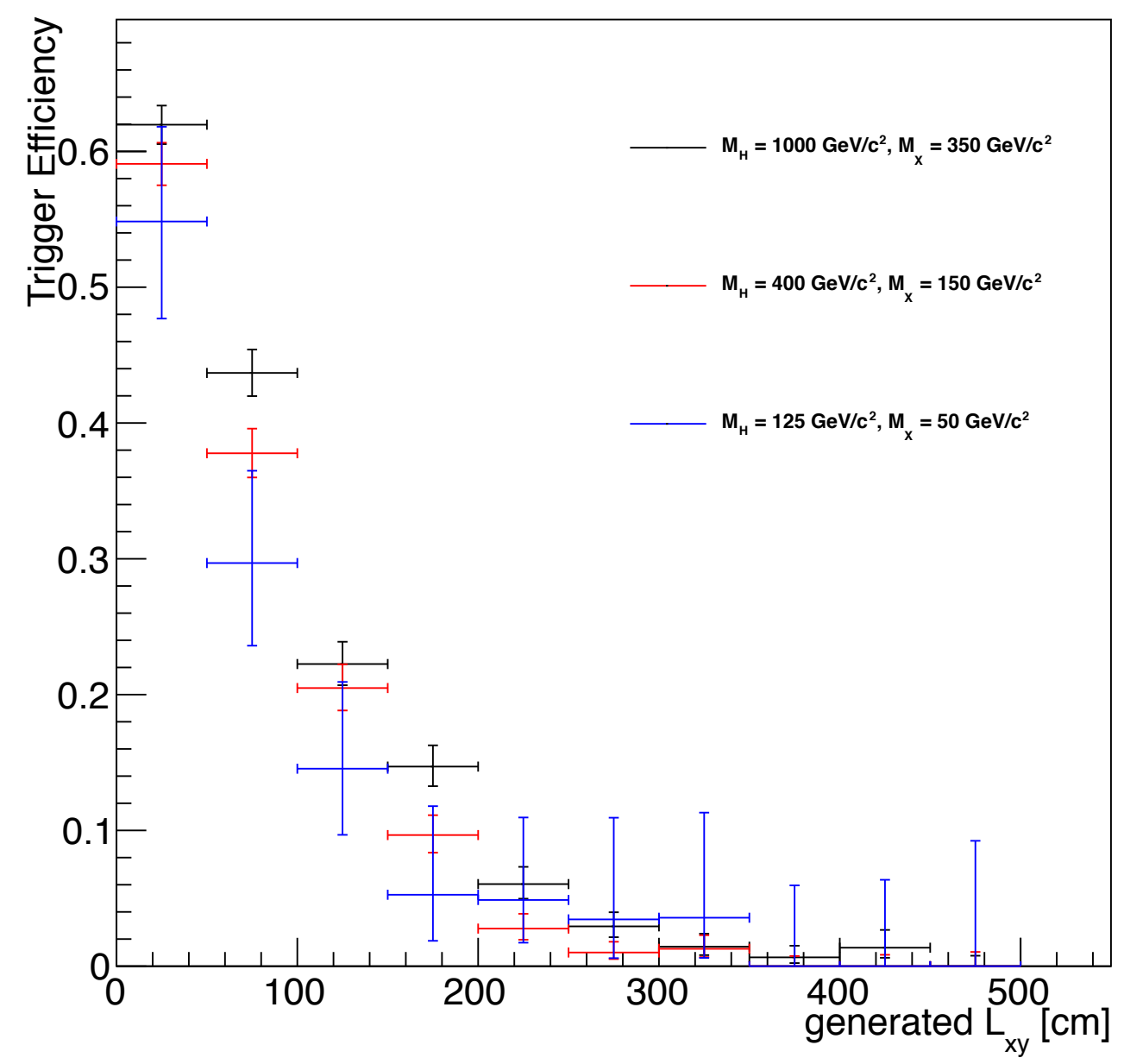

Figure 4.4.: Trigger efficiency vs. generated transverse decay length for the $\mathrm{H}^{0} \rightarrow$ XX signal model with three different mass combinations. The trigger efficiency is computed as the fraction of events within the acceptance that satisfy the trigger requirement. 
In addition, the efficiency of each individual cut in the analysis selection is explicitly shown in Table 4.2. The table contains the cut efficiencies of the signal and background MC samples in the signal region and those of data in the control region. As expected, the cosmic rejection has no effect on the background and signal MC samples, whilst it reduces the background level in data by about a half.

The agreement between background MC and data samples used is shown in Appendix D. The agreement is quite reasonable in the phase space of the analysis, though several minor discrepancies are observed. We use data-driven methods for background estimation in this analysis.
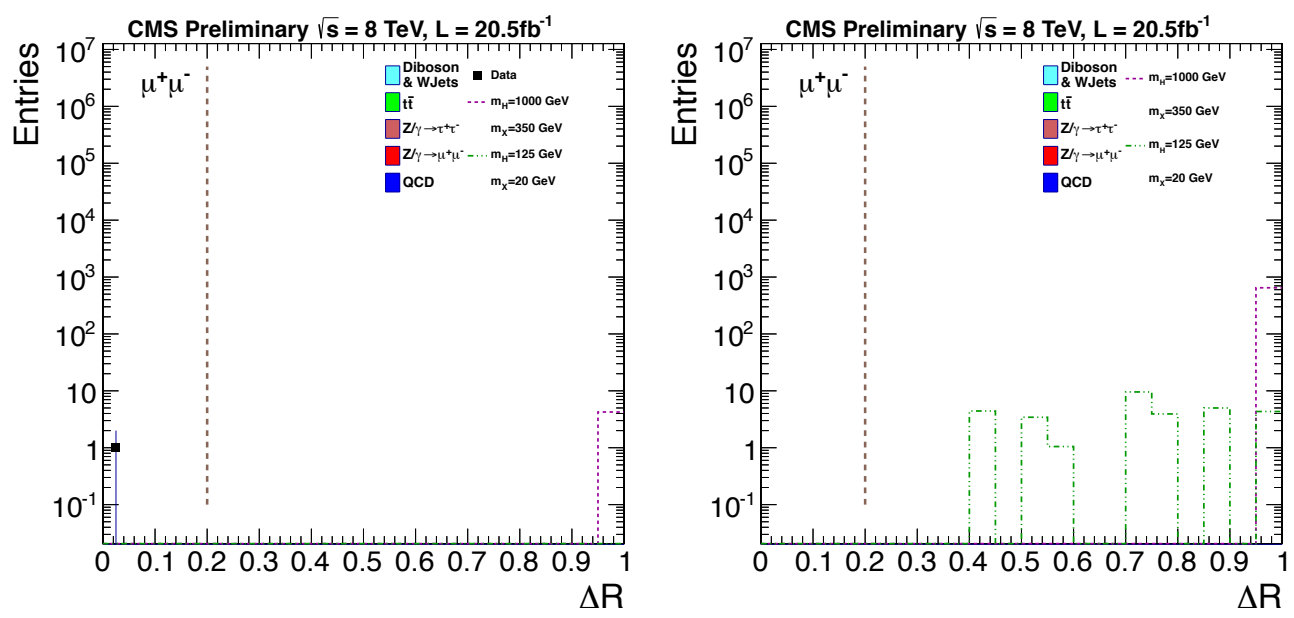

Figure 4.5.: Distribution of $\Delta R$ separation between the two muons for the dimuon candidates passing the full selection except the one plotted in the control region, $|\Delta \Phi|>\pi / 2$ (left) and in the blinded signal region, $|\Delta \Phi|<\pi / 2$ (right). The dashed lines indicate the cut value of the parameter shown.

\subsection{Selection efficiency and acceptance}

The selection efficiency and the limits are determined in terms of the number of events passing our selection, rather than the number of the dimuon candidates. The 

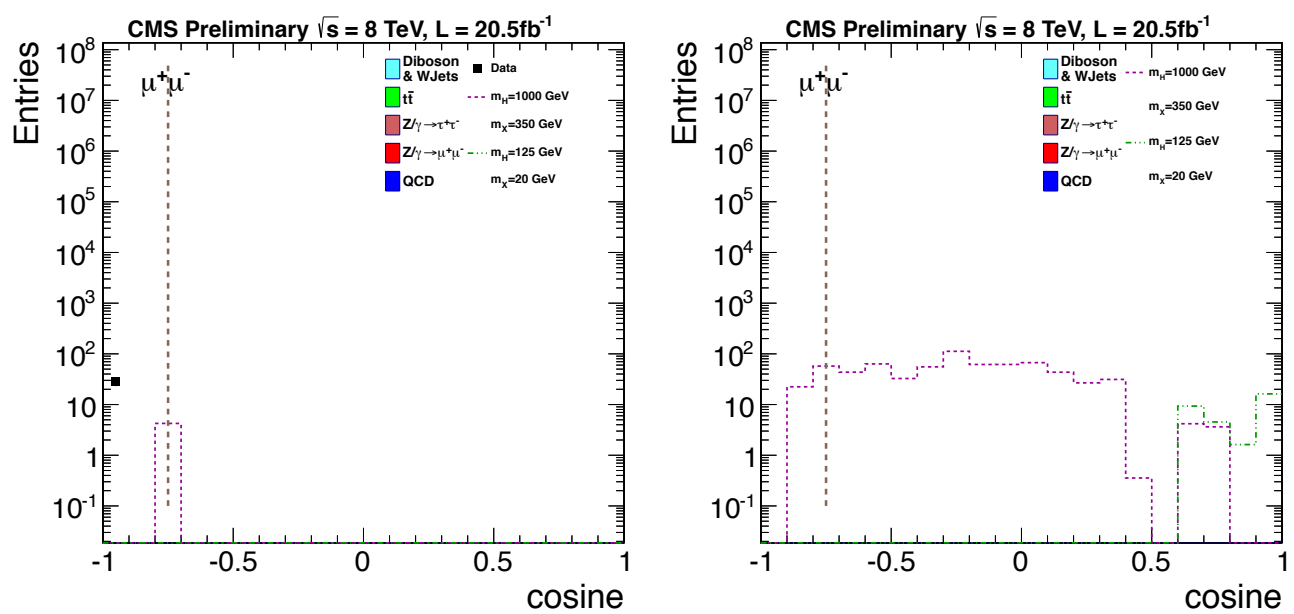

Figure 4.6.: Distribution of the cosine of the 3D opening angle, $\cos (\alpha)$, between the two muons for the dimuon candidates passing the full selection except the one plotted in the control region, $|\Delta \Phi|>\pi / 2$ (left) and blinded signal region, $|\Delta \Phi|<\pi / 2$ (right).
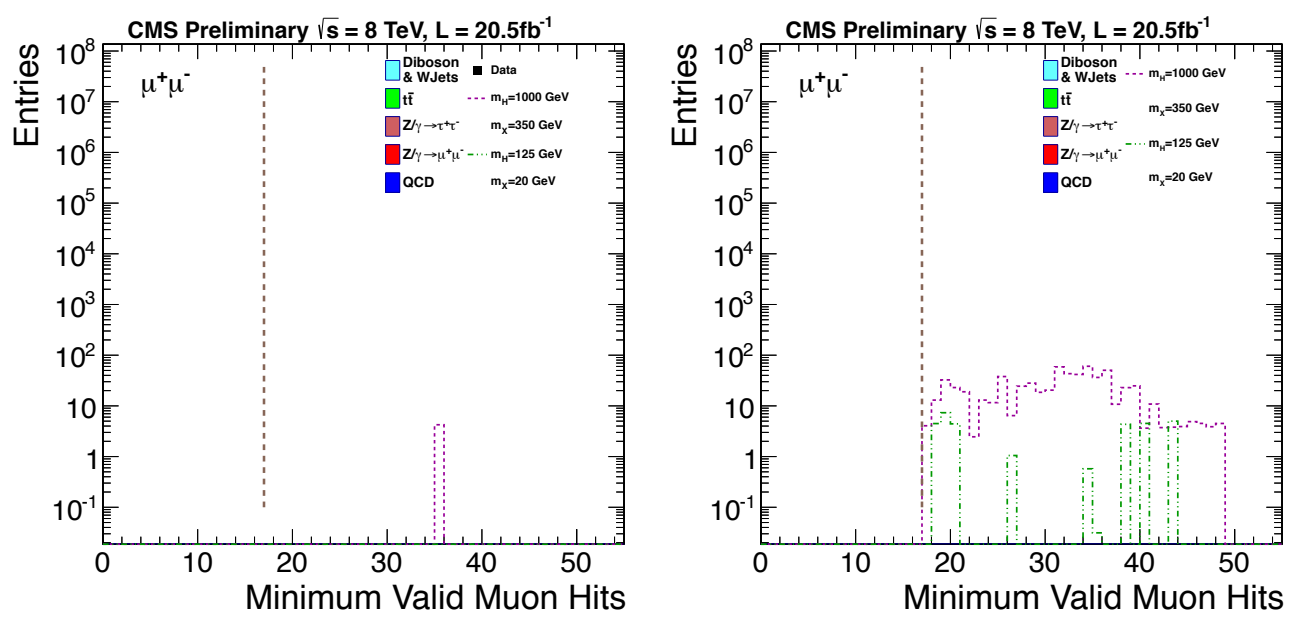

Figure 4.7.: Distribution of the minimum number of valid muon hits of the two muons for the dimuon candidates passing the full selection except the one plotted in the control region, $|\Delta \Phi|>\pi / 2$ (left) and in the blinded signal region, $|\Delta \Phi|<\pi / 2$ (right). 

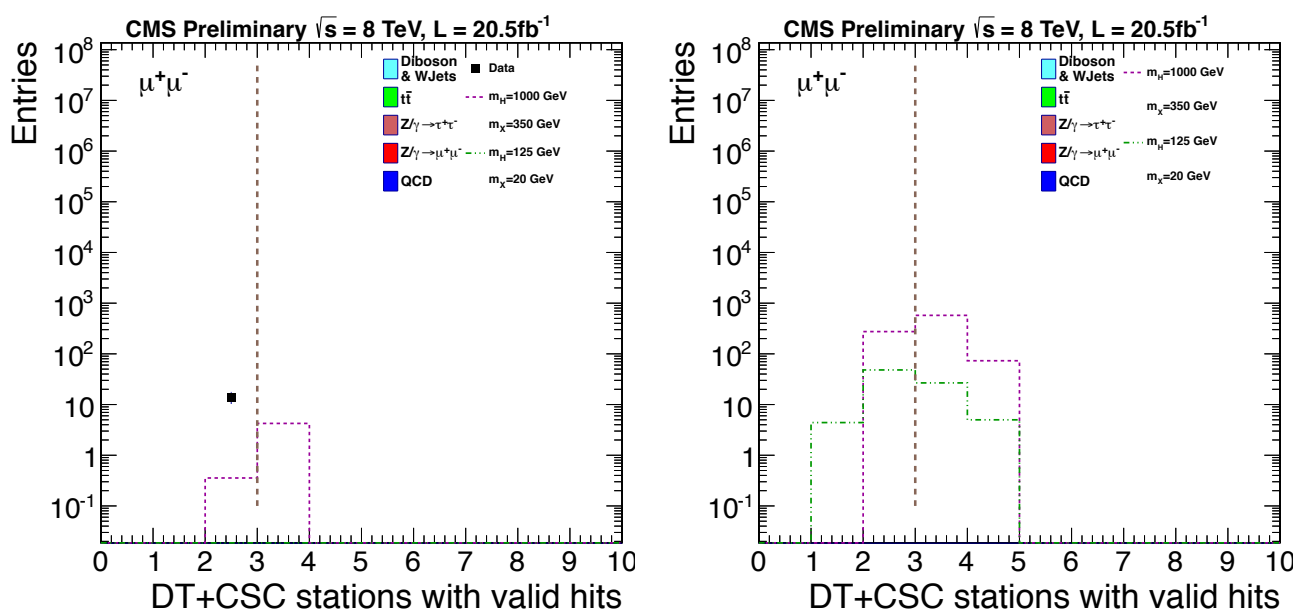

Figure 4.8.: Distribution of the minimum number of valid muon stations of the two muons for the dimuon candidates passing the full selection except the one plotted in the control region, $|\Delta \Phi|>\pi / 2$ (left) and in the blinded signal region, $|\Delta \Phi|<\pi / 2$ (right).
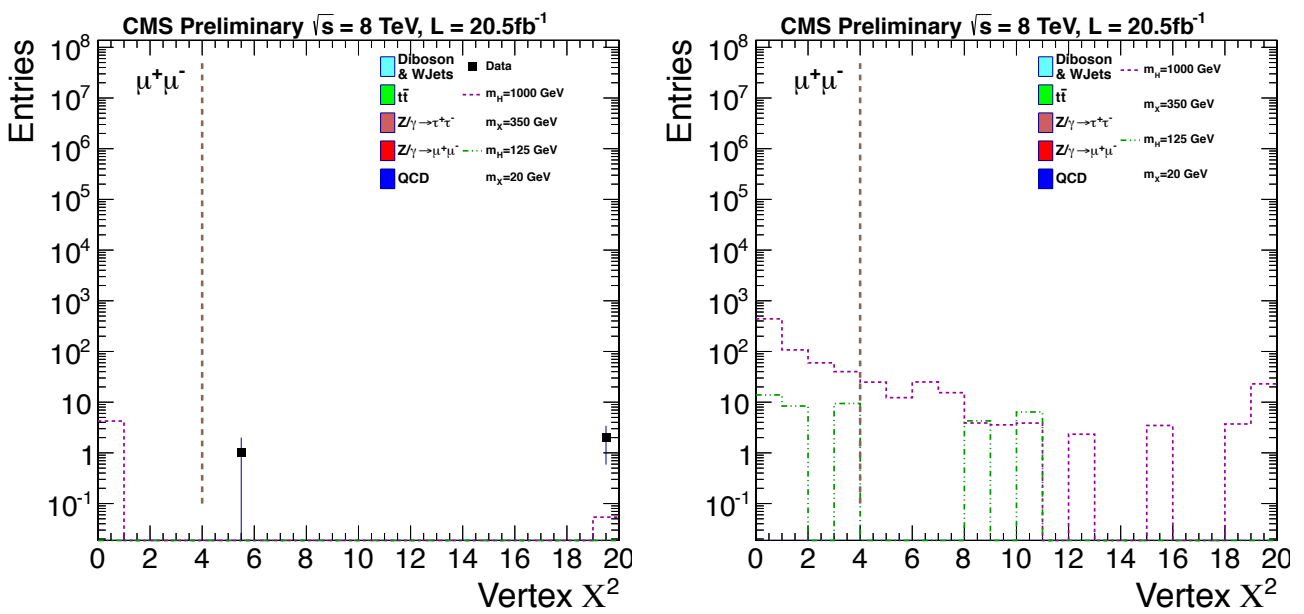

Figure 4.9.: Distribution of the normalized vertex $\chi^{2}$ of the dimuon candidates passing the full selection except the one plotted in the control region, $|\Delta \Phi|>\pi / 2$ (left) and in the blinded signal region, $|\Delta \Phi|<\pi / 2$ (right). 

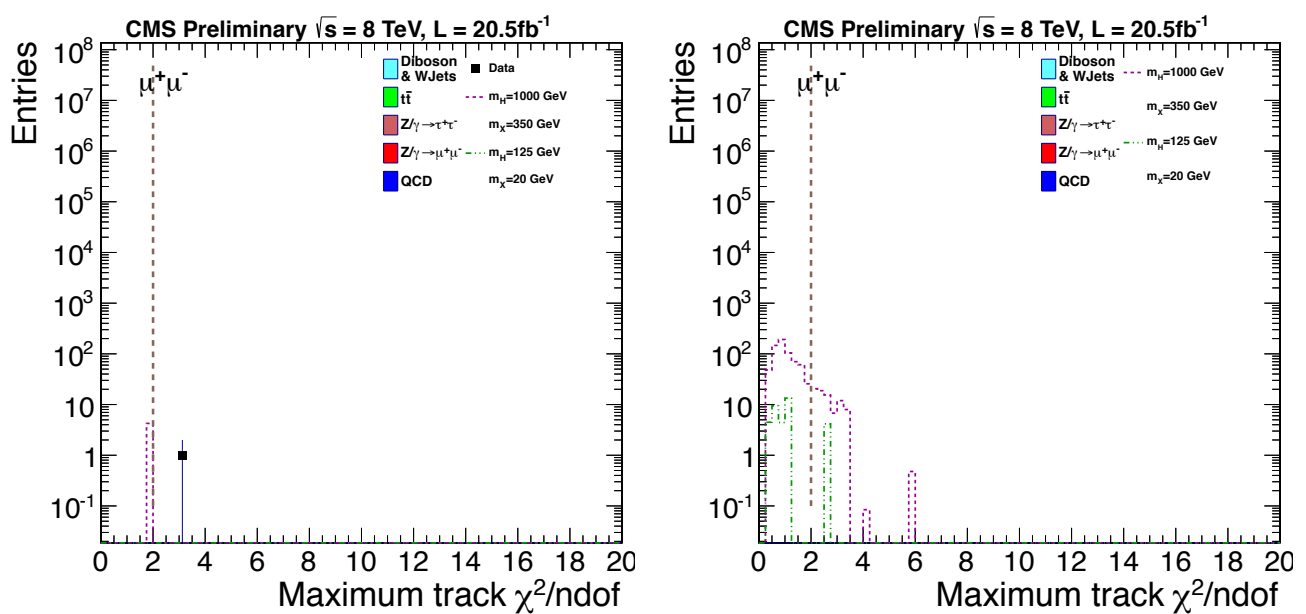

Figure 4.10.: Distribution of the maximum normalized track $\chi^{2}$ of the two muons for the dimuon candidates passing the full selection except the one plotted in the control region, $|\Delta \Phi|>\pi / 2$ (left) and in the blinded signal region, $|\Delta \Phi|<\pi / 2$ (right).
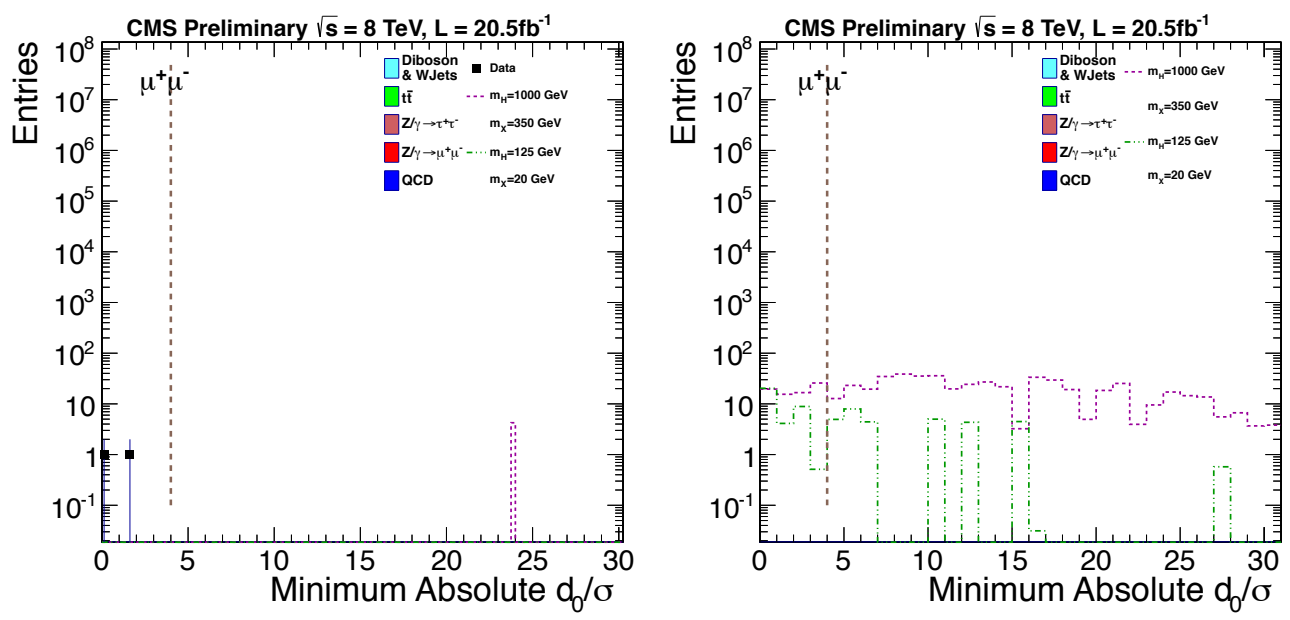

Figure 4.11.: Distribution of the minimum absolute transverse impact parameter significance of the two muons for the dimuon candidates passing the full selection except the one plotted in the control region, $|\Delta \Phi|>\pi / 2$ (left) and in the blinded signal region, $|\Delta \Phi|<\pi / 2$ (right). 

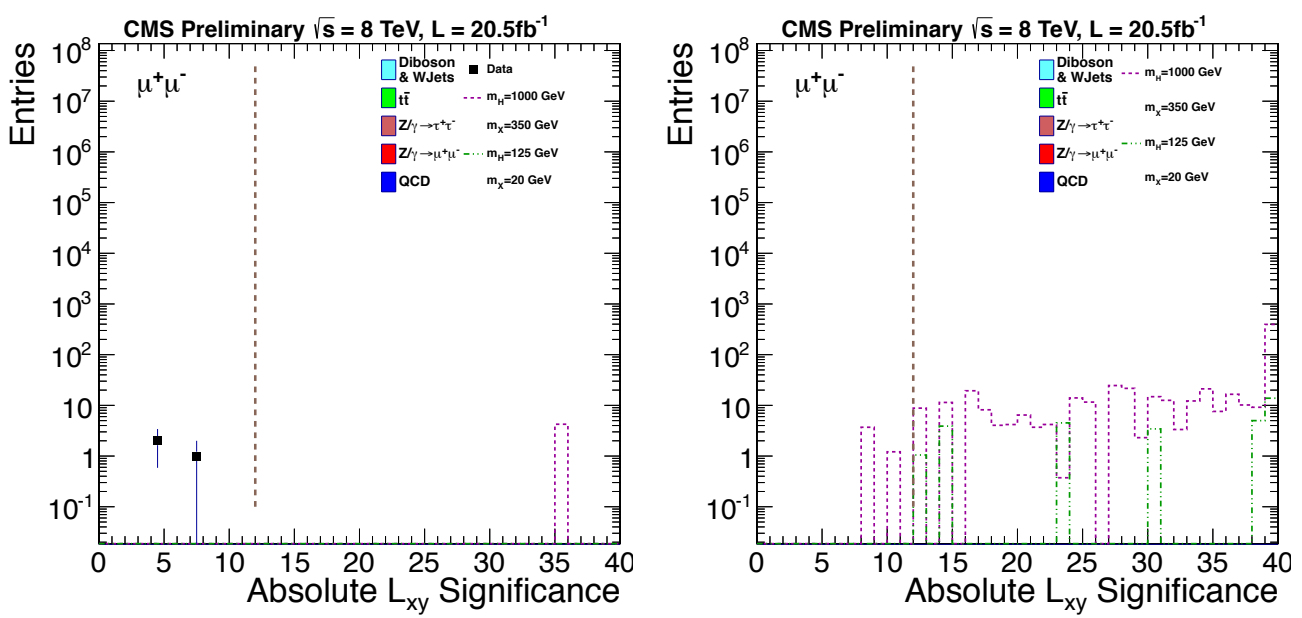

Figure 4.12.: Distribution of the transverse decay length significance of the dimuon candidates passing the full selection except the one plotted in the control region, $|\Delta \Phi|>\pi / 2$ (left) and in the blinded signal region, $|\Delta \Phi|<\pi / 2$ (right). 
Table 4.2.: Dimuon candidate selection efficiencies. Each cut efficiency is the fraction of the candidates passing the cut given that the previous ones are already passed. Preselection is a cut that requires the presence of at least two muons with a transverse momentum, $p_{\mathrm{T}}>26 \mathrm{GeV} / c$ in a triggered event. The cut efficiencies of the signal samples are shown for the events in which there is only one LL particle generated decaying to muons.

\begin{tabular}{|c|c|c|c|c|}
\hline \multirow[b]{2}{*}{ Selection Cut } & \multicolumn{4}{|c|}{ Cut Efficiency } \\
\hline & Data (Control Region) & Background MC & $\mathrm{H}^{0} \rightarrow \mathrm{XX}(1000 / 350)$ & $\mathrm{H}^{0} \rightarrow \mathrm{XX}(125 / 20)$ \\
\hline Trigger & - & - & $26.5 \%$ & $26.8 \%$ \\
\hline Preselection & - & - & $68.9 \%$ & $49.4 \%$ \\
\hline $\begin{array}{l}\text { Muon Matched to Tracker } \\
\text { Tracks }\end{array}$ & $2.8 \%$ & $0.7 \%$ & $56.3 \%$ & $65.1 \%$ \\
\hline Vertex $\chi^{2}$ & $67.6 \%$ & $13.8 \%$ & $85.7 \%$ & $84.9 \%$ \\
\hline Track $\chi^{2}$ & $58.3 \%$ & $65.7 \%$ & $90.6 \%$ & $91.1 \%$ \\
\hline Muon $p_{\mathrm{T}}$ & $100.0 \%$ & $100.0 \%$ & $100.0 \%$ & $100.0 \%$ \\
\hline Muon $|\eta|$ & $72.1 \%$ & $74.8 \%$ & $100.0 \%$ & $100.0 \%$ \\
\hline Dimuon Mass & $93.3 \%$ & $78.7 \%$ & $100.0 \%$ & $96.5 \%$ \\
\hline cos(angle between muons) & $31.8 \%$ & $97.5 \%$ & $94.2 \%$ & $100.0 \%$ \\
\hline$\Delta \mathrm{R}$ & $82.8 \%$ & $100.0 \%$ & $100.0 \%$ & $100.0 \%$ \\
\hline$|\Delta \Phi|$ & $52.2 \%$ & $64.1 \%$ & $97.4 \%$ & $100.0 \%$ \\
\hline $\begin{array}{l}\text { Contamination with Cos- } \\
\text { mics }\end{array}$ & $55.1 \%$ & $99.0 \%$ & $96.0 \%$ & $100.0 \%$ \\
\hline $\begin{array}{l}\text { Min. Number of DT + CSC } \\
\text { Stations }\end{array}$ & $16.6 \%$ & $12.1 \%$ & $53.0 \%$ & $54.7 \%$ \\
\hline $\begin{array}{l}\text { Min. Number of Valid } \\
\text { Muon Hits }\end{array}$ & $96.9 \%$ & $100.0 \%$ & $100.0 \%$ & $100.0 \%$ \\
\hline Muon $\left|d_{0}\right| / \sigma_{d}$ & $0.7 \%$ & $0.0 \%$ & $83.9 \%$ & $25.9 \%$ \\
\hline Dimuon $L_{x y} / \sigma_{L_{x y}}$ & $0.0 \%$ & $0.0 \%$ & $99.2 \%$ & $100.0 \%$ \\
\hline
\end{tabular}


full signal efficiency is simply the ratio of the total number of generated events to the number of events in which at least a LL candidate passes the full selection. It is computed separately for two different cases. In the first case, the events that have only one generated LL particle $(\mathrm{X})$ decaying to muons give the efficiency $\epsilon_{1}$; whereas the efficiency for the events in which two generated LL particles decay to muons is denoted by $\epsilon_{2}$. The efficiencies are estimated by reweighting the generated events with respect to the generated lifetimes. The efficiencies of the signal samples with the longest lifetime to pass the full selection of the analysis are tabulated in Table 4.3. In addition, the efficiencies of the simulated events that are within acceptance, as described in Chapter 4.2, to satisfy the selection criteria is given in Table 4.4.

Table 4.3.: Efficiencies of the signal MC samples to pass the full selection of the analysis for the events where only one simulated LL particle decays to muons $\left(\epsilon_{1}\right)$, and for the events where two generated LL particles decay to muon pairs $\left(\epsilon_{2}\right)$.

\begin{tabular}{|c|c|c||c|c|}
\hline$M_{\mathrm{H}^{0}}$ & $M_{\mathrm{X}}$ & $c \tau$ & \multicolumn{2}{|c|}{ Efficiency } \\
\hline$\left(\mathrm{GeV} / c^{2}\right)$ & $\left(\mathrm{GeV} / c^{2}\right)$ & $(\mathrm{cm})$ & $\epsilon_{1}$ & $\epsilon_{2}$ \\
\hline 1000 & 350 & 350 & 0.023 & 0.044 \\
\hline 1000 & 150 & 100 & 0.045 & 0.076 \\
\hline 1000 & 50 & 40 & 0.018 & 0.022 \\
\hline 1000 & 20 & 15 & 0.0015 & 0.0017 \\
\hline 400 & 150 & 400 & 0.015 & 0.04 \\
\hline 400 & 50 & 80 & 0.030 & 0.053 \\
\hline 400 & 20 & 40 & 0.0094 & 0.013 \\
\hline 200 & 50 & 200 & 0.0084 & 0.019 \\
\hline 200 & 20 & 70 & 0.0068 & 0.012 \\
\hline 125 & 50 & 500 & 0.0018 & 0.0042 \\
\hline 125 & 20 & 130 & 0.0011 & 0.0027 \\
\hline
\end{tabular}


Finally, the full selection is also implemented on the cosmics enriched sample to test how effective the analysis is to reject the events with cosmic origin. The analysis is run over the two cosmics datasets taken in 2012 between the run periods, Run B-Run C and Run C-Run D, at the LHC. The datasets contain 13 million events triggered by the dedicated cosmic muon trigger. We reconstruct 160,000 events consisting of dimuons. Only one of these events passes the analysis selection, corresponding to an efficiency of $0.000625 \%$ which is about 200 times smaller than the lowest signal efficiency of the analysis. Therefore, the events originating from cosmics are suppressed by the analysis.

Table 4.4.: Efficiencies of the signal MC samples to satisfy the full selection for events within the acceptance. The efficiencies are computed separately for the events where only one simulated LL particle decays to muons $\left(\epsilon_{1}\right)$, and for the events where two generated LL particles decay to muon pairs $\left(\epsilon_{2}\right)$.

\begin{tabular}{|c|c|c||c|c|}
\hline$M_{\mathrm{H}^{0}}$ & $M_{\mathrm{X}}$ & $c \tau$ & \multicolumn{2}{c|}{ Efficiency } \\
\hline$\left(\mathrm{GeV} / c^{2}\right)$ & $\left(\mathrm{GeV} / c^{2}\right)$ & $(\mathrm{cm})$ & $\epsilon_{1}$ & $\epsilon_{2}$ \\
\hline 1000 & 350 & 350 & 0.032 & 0.064 \\
\hline 1000 & 150 & 100 & 0.066 & 0.12 \\
\hline 1000 & 50 & 40 & 0.029 & 0.031 \\
\hline 1000 & 20 & 15 & 0.0019 & 0.0017 \\
\hline 400 & 150 & 400 & 0.025 & 0.072 \\
\hline 400 & 50 & 80 & 0.061 & 0.12 \\
\hline 400 & 20 & 40 & 0.02 & 0.034 \\
\hline 200 & 50 & 200 & 0.033 & 0.11 \\
\hline 200 & 20 & 70 & 0.027 & 0.07 \\
\hline 125 & 50 & 500 & 0.035 & 0.1 \\
\hline 125 & 20 & 130 & 0.0091 & 0.32 \\
\hline
\end{tabular}




\section{CHAPTER 5. ESTIMATED BACKGROUND AND ASSOCIATED SYSTEMATIC UNCERTAINTIES}

The signal and the background have a different distribution in $|\Delta \Phi|$. The signal is expected to have small $|\Delta \Phi|$ values, while the background distribution is expected to be uniform in $|\Delta \Phi|$ due to the absence of a genuine secondary vertex, as shown in Figure 5.1.
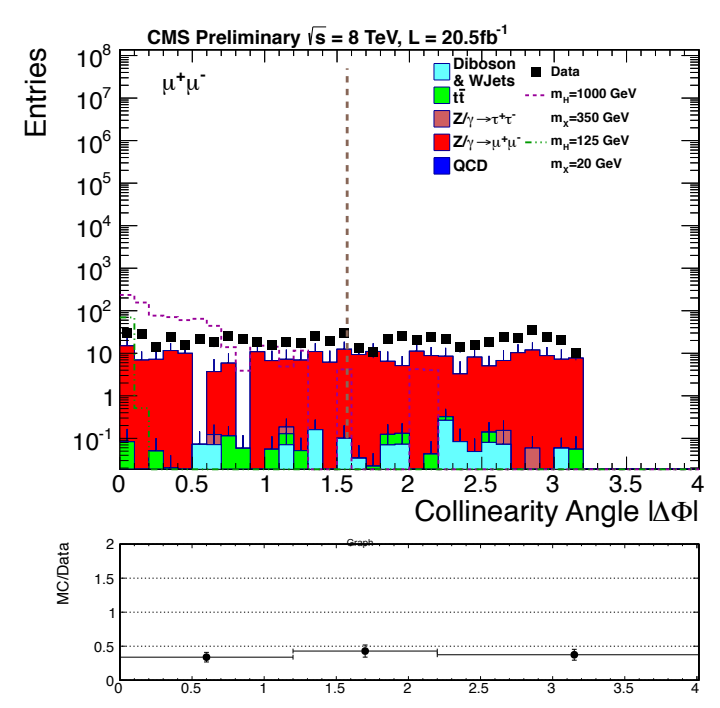

Figure 5.1.: Collinearity angle, $|\Delta \Phi|$, distribution for the dimuon candidates passing the full selection except $\left|d_{0}\right| / \sigma_{d}, L_{x y} / \sigma_{L_{x y}}$ and $|\Delta \Phi|$ cuts. The signal events plotted have only one LL particle generated decaying to muons. The dashed line indicates the cut value of the parameter shown.

No data events are observed after the full selection is applied in the control region. Signal-control region symmetry sets the nominal value of the background expectation in the signal region to zero as well. The systematic uncertainty on this estimate is computed in Chapter 5.2. 


\subsection{Background validation}

The background symmetry is confirmed for data by comparing the $L_{x y} / \sigma_{L_{x y}}$ tailcumulative distribution in the signal region with that in the control region at modest $L_{x y} / \sigma_{L_{x y}}$ and $\left|d_{0}\right| / \sigma_{d}$ values where the data is background-dominated. Similarly, the study is also repeated using simulated background events, though the distribution is not expected to have similar normalization as in data since background MC samples do not fully describe the data. For both studies, the full selection except the $L_{x y} / \sigma_{L_{x y}}$ cut is implemented and the $\left|d_{0}\right| / \sigma_{d}$ cut is reversed, $\left|d_{0}\right| / \sigma_{d}<4$. The $L_{x y} / \sigma_{L_{x y}}$ tailcumulative plot for data excludes the region $L_{x y} / \sigma_{L_{x y}}>6$ where potential signal events might appear.
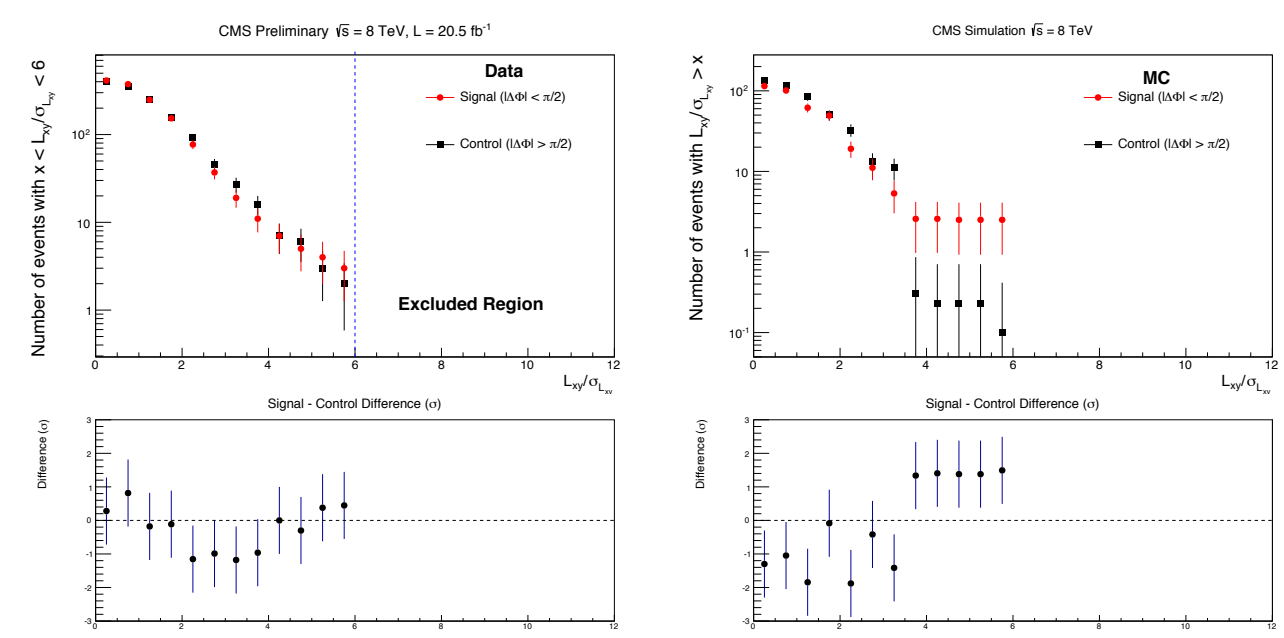

Figure 5.2.: Comparisons of $L_{x y} / \sigma_{L_{x y}}$ tail-cumulative distributions between signal ,$|\Delta \Phi|<\pi / 2$, and control, $|\Delta \Phi|>\pi / 2$, regions for both data (upper left) and background MC samples (upper right). The full selection is applied with the exception of the $L_{x y} / \sigma_{L_{x y}}$ cut. The $\left|d_{0}\right| / \sigma_{d}$ cut is reversed to $\left|d_{0}\right| / \sigma_{d}<4$. The plots on the bottom left and the bottom right show the statistical significance of the difference between the two regions for data and $\mathrm{MC}$, respectively. The $L_{x y} / \sigma_{L_{x y}}>6$ region is excluded in data to avoid the possible signal contamination. 
Figure 5.2 shows the tail-cumulative distributions (i.e., the integral from the cut value on the horizontal axis to infinity) of $L_{x y} / \sigma_{L_{x y}}$ in the signal and control regions for both data and simulated background events. Additionally, the discrepancy between control and signal regions is expressed in terms of the combined uncertainty for each bin. The difference is found to be in agreement with the symmetric background hypothesis.

\subsection{Background systematic uncertainties}

There are three main classes of systematic uncertainty in this analysis. These are the uncertainty on the integrated luminosity, the uncertainty in the signal selection, which will be discussed in Chapter 6, and the uncertainty that arises when deriving the background estimate. To derive a systematic uncertainty on the estimated background from data, a fit to the $L_{x y} / \sigma_{L_{x y}}$ distribution is performed in a background dominated region. The fit function is extrapolated to the signal region and is used to obtain an estimated background. The difference of this estimate, plus its uncertainty, from the nominal estimated background of zero events is taken as systematic uncertainty. However, as there is not enough statistic to perform a meaningful fit of the background shape after the full selection, shown in the right plot of Figure 5.3, we remove the track rejection cut and fit the resulting $L_{x y} / \sigma_{L_{x y}}$ distribution, shown in the left plot of Figure 5.3. The shape of the distribution is due to the $\left|d_{0}\right| / \sigma_{d}$ cut. Since we are only interested in modelling the shape of the right tail of the distribution, we perform a fit using a simple exponential of the form $A e^{-\alpha L_{x y} / \sigma_{L_{x y}}}$ and we only fit the region $L_{x y} / \sigma_{L_{x y}}>7$. The resulting fit is represented by the red curve in the figure.

Furthermore, it can be also inferred from Figure 5.3 that there is only one event with $L_{x y} / \sigma_{L_{x y}}>7$ in the control region of the data. Under the assumption above, the parameter $A$ can be rescaled such that the overall function is normalized to unity since there is one event in the region of the fit. Finally, the number of estimated 

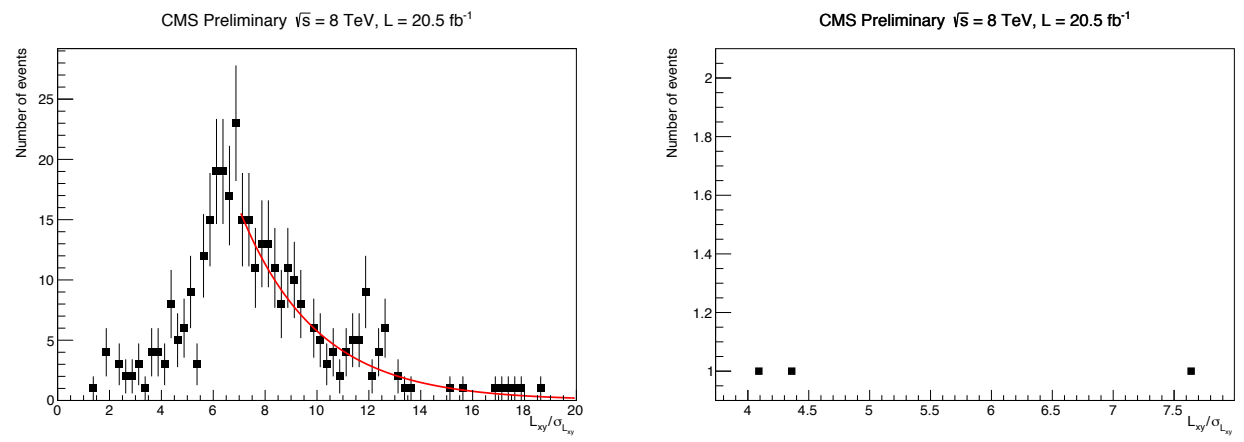

Figure 5.3.: (Left) $L_{x y} / \sigma_{L_{x y}}$ distribution after applying the full selection except the $L_{x y} / \sigma_{L_{x y}}$ and the tracker track rejection cuts on the data in the control region. The shape is largely determined by the $\left|d_{0}\right| / \sigma_{d}$ cut. The red curve shows the exponential fit for $L_{x y} / \sigma_{L_{x y}}>7$ region. (Right) $L_{x y} / \sigma_{L_{x y}}$ distribution after applying the full selection except the $L_{x y} / \sigma_{L_{x y}}$ cut. Only one event is found with $L_{x y} / \sigma_{L_{x y}}$ larger than 7 .

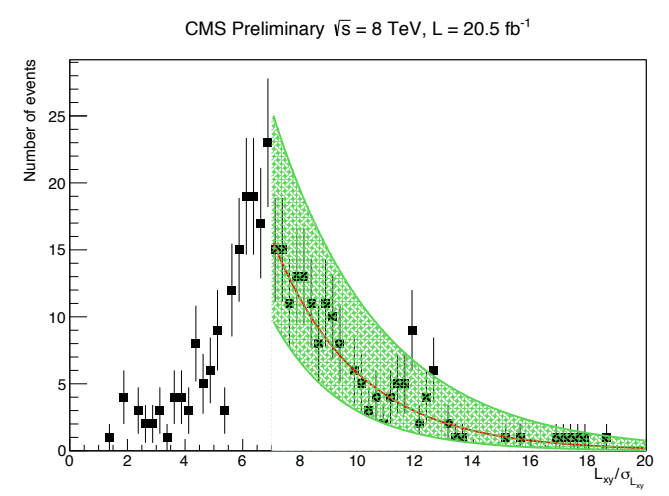

Figure 5.4.: $L_{x y} / \sigma_{L_{x y}}$ distribution after applying the full selection except the $L_{x y} / \sigma_{L_{x y}}$ and the tracker track rejection cuts on the data in the control region. The shape is largely determined by the $\left|d_{0}\right| / \sigma_{d}$ cut. The green band shows the variation in the fitted background shape when the exponential fit parameter $\alpha$ is varied by $\pm 20 \%$. 
background events from this method can be calculated by integrating the rescaled function between 12 and infinity. Back-of-the-envelope calculation yields $0.18 \pm 0.03$.

We assign a systematic uncertainty to the shape determination by varying the slope parameter, $\alpha$, by $\pm 20 \%$. Figure 5.4 illustrates the variation in the background shape, which spans the statistical uncertainty in the background distribution. Combining the background estimate above and the variational effect in the background shape gives $0.18 \pm 0.03(\text { stat })_{-0.07}^{+0.12}$ (syst). We take 0.33 , evaluated as the sum of the central value, the statistical error, and the positive systematic uncertainty from the fit result, as systematic uncertainty on the background estimated from data in the control region. 


\section{CHAPTER 6. SIGNAL SYSTEMATIC UNCERTAINTIES}

The dominant systematic uncertainties in the analysis are associated with the signal efficiency and are caused by uncertainties in the trigger and reconstruction efficiencies of the displaced RSA muons and by the pileup modelling in the simulation. A summary of the sources of systematic uncertainty affecting the signal efficiency is presented in Table 6.1. In addition, we consider the effect of pileup on the cosmic muon and tracker track rejection cuts and the effect of RSA muon $p_{\mathrm{T}}$ resolution. They appear to be negligible and we do not assign any additional systematic uncertainty, as detailed later.

Table 6.1.: Systematic uncertainties related to the signal selection. The uncertainty specified is a relative uncertainty. The relative uncertainty in the luminosity is $2.6 \%$.

\begin{tabular}{|r|l|}
\hline Source & Uncertainty \\
\hline Pileup modelling & $2 \%$ \\
Tracking efficiency from cosmics & $18 \%$ \\
Trigger efficiency & $17 \%$ \\
\hline
\end{tabular}

\subsection{Luminosity}

For the running period corresponding to this analysis, CMS estimates the relative uncertainty on the luminosity to be $2.6 \%$ [16]. This uncertainty is used when calculating the final cross section estimates. 


\subsection{Effect of pileup}

In order for the simulation to describe the pileup events in data realistically, the background simulation events are reweighted to match the pileup in data by following the procedure given in Ref. [17]. Data and reweighted background MC events are compared in Figure 6.1 in terms of the number of reconstructed primary vertices, which is an estimate of the pileup.

The systematic uncertainty on the pileup modelling is estimated varying the average number of reconstructed primary vertices in the background $\mathrm{MC}$ events by $\pm 5 \%$ as recommended in Ref. [18]. The variation is realized through the re-reweighting of the simulation. The systematic uncertainty on the signal efficiencies due to the pileup modelling is found to be less than $2 \%$ for all signal MC samples used.

The more collisions that occur during the bunch crossing, the more tracks would be reconstructed by the silicon tracker. This would increase the probability that we reject extra signal events from a mismatch. Therefore, the possible dependence of the track rejection and cosmic rejection cuts on pileup is investigated on signal simulated $\mathrm{H}^{0} \rightarrow \mathrm{XX}$ events with $M_{\mathrm{H}^{0}}=1000 \mathrm{GeV} / c^{2}, M_{\mathrm{X}}=350 \mathrm{GeV} / c^{2}$ and $c \tau=350 \mathrm{~cm}$. The two cut efficiencies vs. the number of reconstructed primary vertices are plotted in Figure 6.2 by applying the rest of the analysis selection. Due to the limited statistics, a solid conclusion could not be drawn. Figure 6.3 shows the pileup independence of the two cuts when the $\left|d_{0}\right| / \sigma_{d}$ and $L_{x y} / \sigma_{L_{x y}}$ cuts are removed from the full selection and the minimum valid muon station requirement is loosened from 3 to 2 .

\subsection{Track finding and selection efficiency}

To assess if the efficiency to reconstruct displaced muons in the muon chambers is correctly modeled by the simulation, a direct measurement is performed utilizing cosmic ray muons. Events are selected from dedicated runs with no beam activity (CRAFT) and the cosmic ray muons are reconstructed as two separate RSA muons

in opposite halves of the CMS detector. The trigger used to collect the events during 


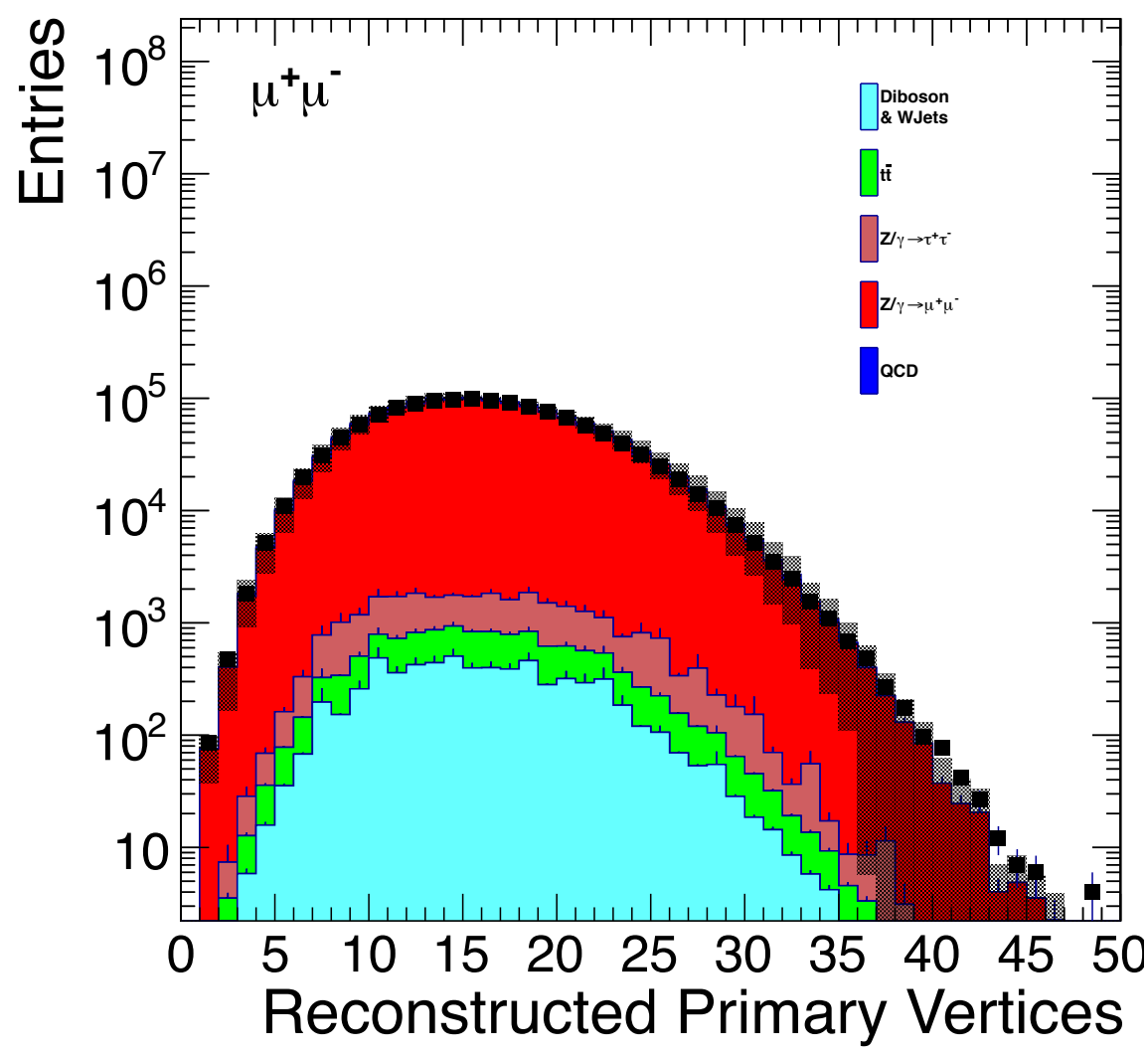

Figure 6.1.: Distribution of the number of reconstructed primary vertices for data and the background simulation. The simulation is reweighted according to the procedure explained in the text. The full selection except the tracker track rejection and the cuts on $\left|d_{0}\right| / \sigma_{d}$ and $L_{x y} / \sigma_{L_{x y}}$ is applied. The grey vertical band represents the systematic uncertainty from varying the pileup weights by $\pm 5 \%$. 

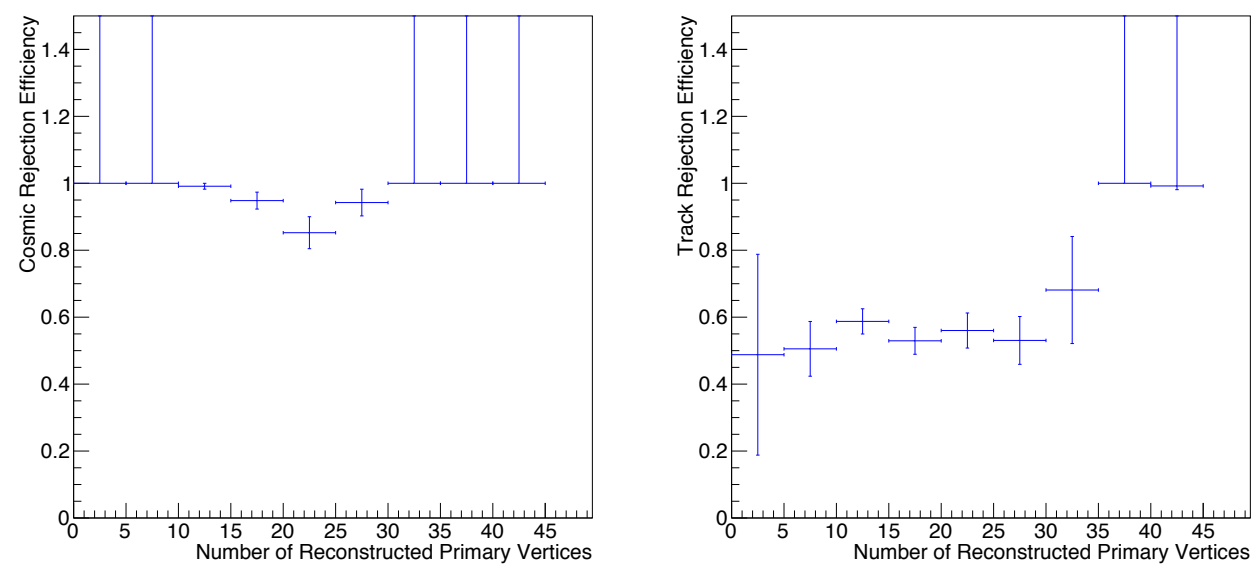

Figure 6.2.: Efficiency of the cosmic muon rejection (left) and of the tracker track rejection (right) vs. the number of reconstructed primary vertices for the signal MC sample with $M_{\mathrm{H}^{0}}=1000 \mathrm{GeV} / c^{2}, M_{\mathrm{X}}=350 \mathrm{GeV} / c^{2}$ and $c \tau=350 \mathrm{~cm}$. All other analysis selection cuts are applied. In both cases no significant dependence on the number of reconstructed primary vertices is observed. 

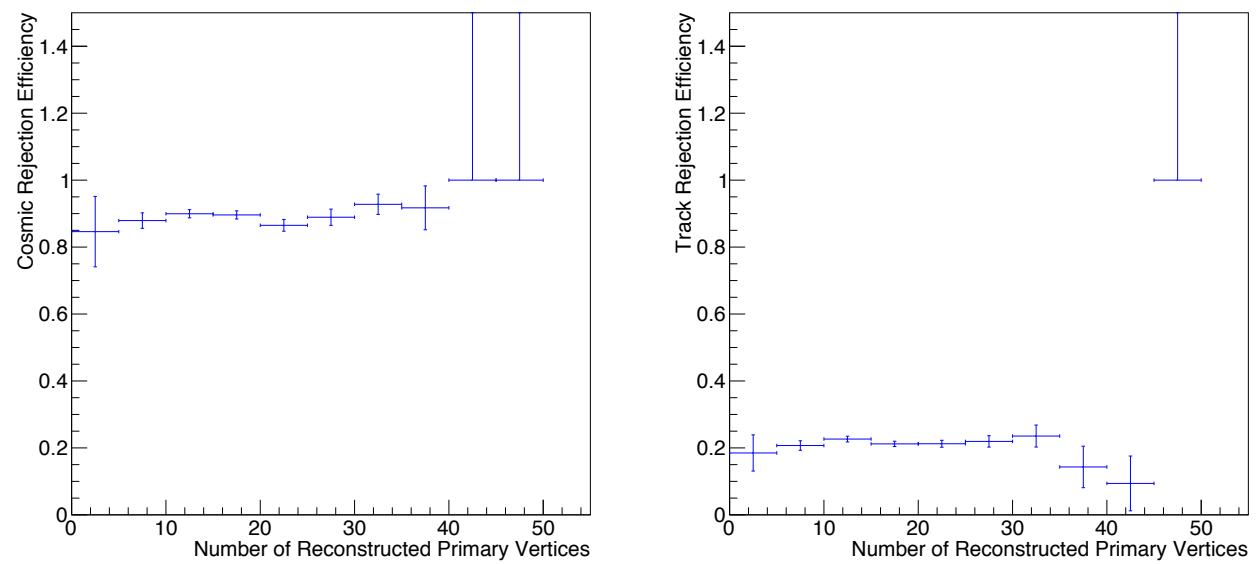

Figure 6.3.: Efficiency of the cosmic muon rejection (left) and of the tracker track rejection (right) vs. the number of reconstructed primary vertices for the signal MC sample with $M_{\mathrm{H}^{0}}=1000 \mathrm{GeV} / c^{2}, M_{\mathrm{X}}=350 \mathrm{GeV} / c^{2}$ and $c \tau=350 \mathrm{~cm}$. All other analysis selection cuts are applied except the $\left|d_{0}\right| / \sigma_{d}$ and $L_{x y} / \sigma_{L_{x y}}$ cuts. The minimum muon valid station requirement is also loosened for both plots. In both cases no significant dependence on the number of reconstructed primary vertices is observed. 
cosmic runs is a dedicated RPC technical trigger that requires a signal in the RPC in the current and the previous two bunch crossings. This requirement ensures that if a muon is coming from above the detector, it will reach the bottom muon chambers in time with the readout of the detector. In essence, the bottom half of a cosmic muon has the same timing, from the point of view of the detector readout, as a muon coming from a collision. The same trigger is not available for the simulation. However, by generating cosmic muons with a production time delayed by $25 \mathrm{~ns}$ we achieve a similar distribution of cosmic muons. The distribution of timing related variables for cosmic data and simulation for all muon candidates is shown in Figure 6.4 and Figure 6.5. The simulation only reproduces the main peak of the timeAtIpInOut for data. This is expected as muons arriving with $25 \mathrm{~ns}$ or $50 \mathrm{~ns}$ before or after the ideal timing in data might still have a chance to pass the trigger while the simulation does not generate such events. To select a consistent sample between data and simulation we require that the muon at the top has a timeAtIpInOut in $[-40,-20] \mathrm{ns}$. This requirement also limits any bias due to timing on the measured efficiency since the bottom half of the cosmic muon is in time with the detector readout.

The timing related variables after this selection for muons in the top half of CMS are shown in Figure 6.6 and Figure 6.7. The comparisons between the kinematic distributions for cosmic data and simulation are shown in Figure 6.8 and Figure 6.9 for all candidate muons and for the selected ones, respectively.

We perform two measurements of the track finding efficiency. The first one is relative to the silicon tracker and the second one uses only the muon chambers. In the first measurement we require at least one track reconstructed in the silicon tracker passing the following selection:

- $p_{T}>26 \mathrm{GeV} / \mathrm{c}$,

- $|\eta|<2$,

- at least 6 valid hits. 

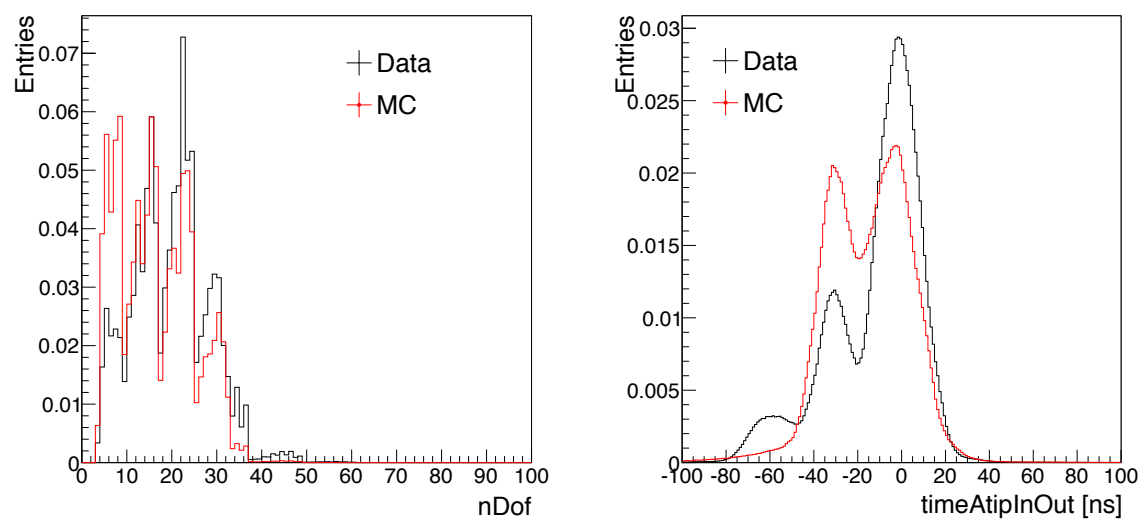

Figure 6.4.: Number of degrees of freedom (left) and timeAtIpInOut (right) in cosmic data and simulation for all muon candidates.
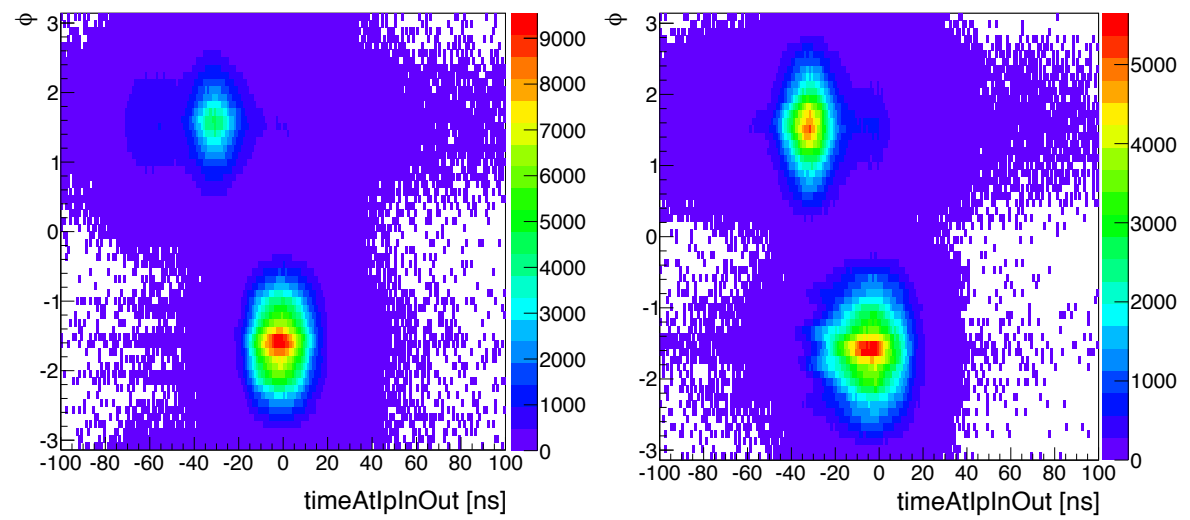

Figure 6.5.: timeAtIpInOut vs $\phi$ for data (left) and MC (right) for all muon candidates. 

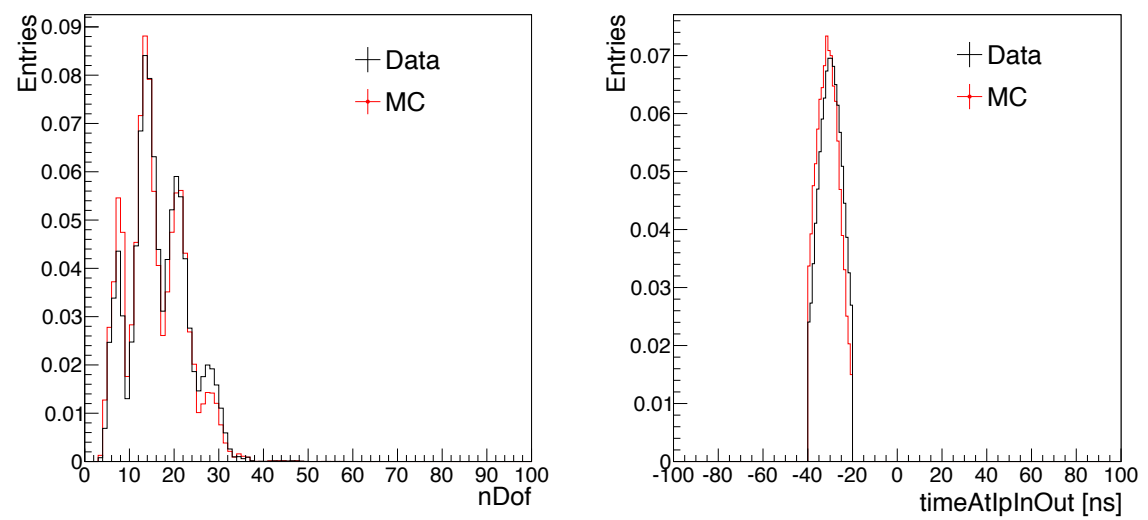

Figure 6.6.: Number of degrees of freedom (left) and timeAtIpInOut (right) in cosmic data and simulation for muon candidates in the top half of CMS passing the timing selection.
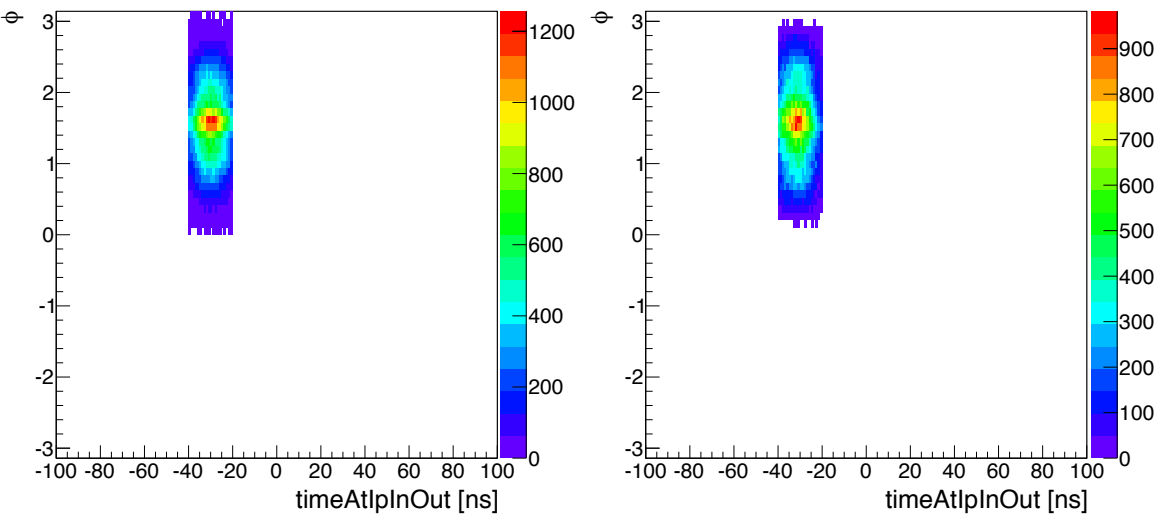

Figure 6.7.: timeAtIpInOut vs $\phi$ for data (left) and MC (right) for muon candidates in the top half of CMS passing the timing selection. 

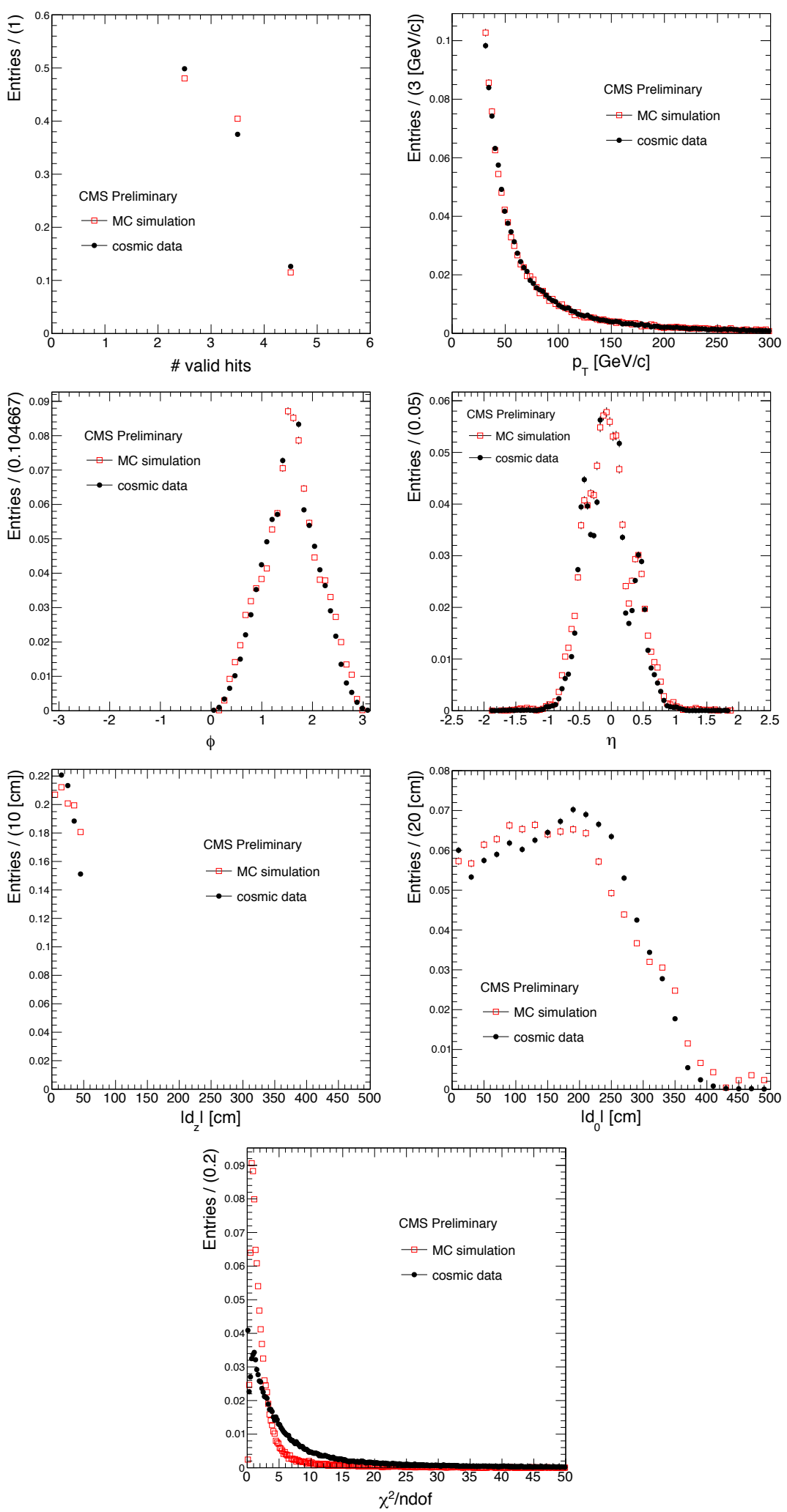

Figure 6.8.: Comparison of kinematic distributions in cosmic data and simulation for all muon candidates. 

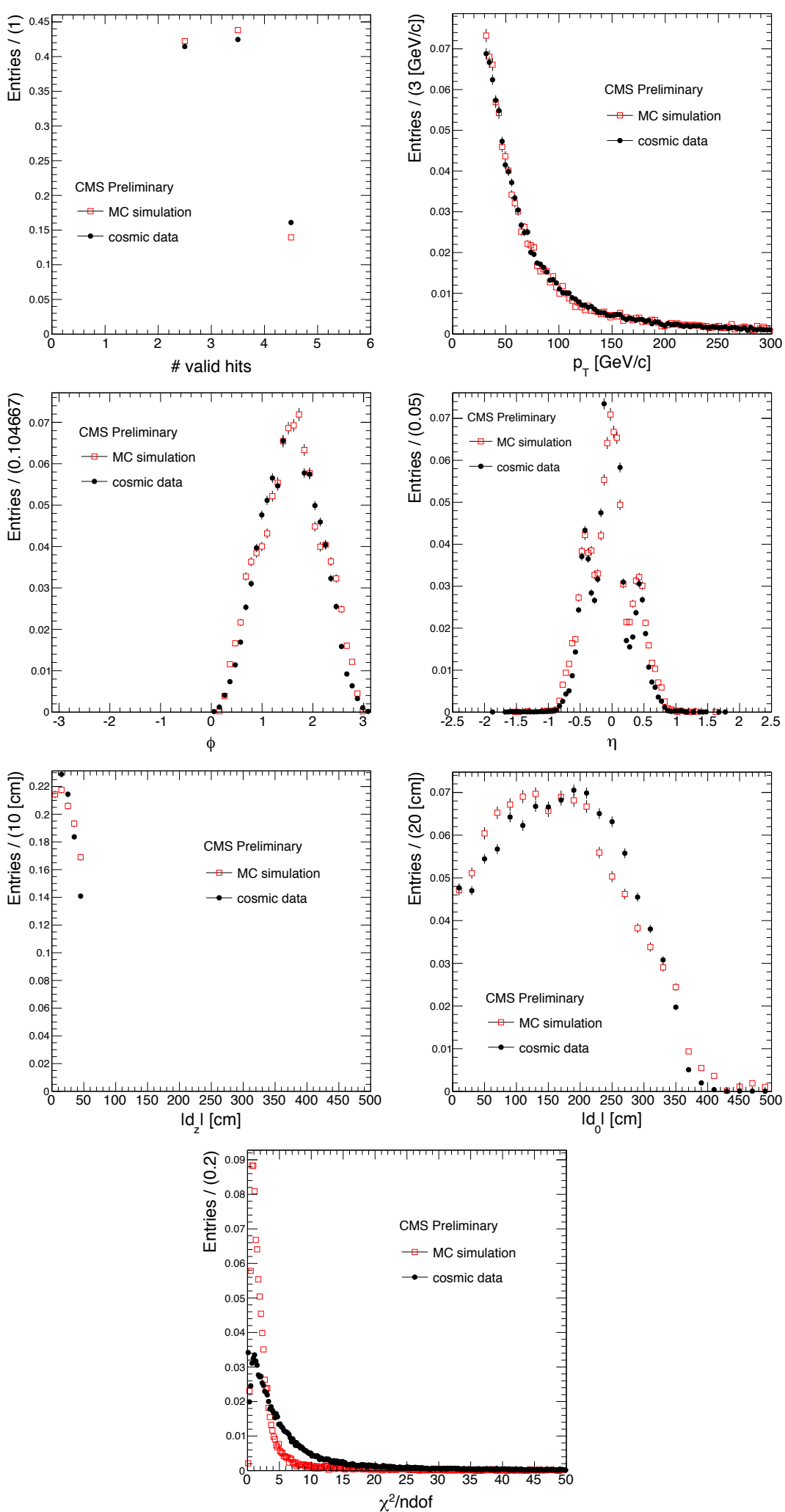

Figure 6.9.: Comparison of kinematic distributions in cosmic data and simulation for muon candidates passing the selection detailed in the text. 
The efficiency is defined as the ratio of the number of reconstructed RSA muons in the bottom half of CMS to the total number of tracks found in the silicon tracker. The RSA muons must additionally satisfy the following criteria:

- $p_{T}>26 \mathrm{GeV} / \mathrm{c}$,

- $|\eta|<2$,

- at least 17 valid hits in the DT or CSC muon chambers,

- at least one valid hit in three DT or CSC stations.

The result is shown in Figure 6.10 as a function of the transverse impact parameter of the track. By construction, this method is only sensitive to the impact parameters up to a few tens of centimeters since the track finding efficiency for the silicon tracker is zero for higher values of the impact parameters.

The second measurement allows to explore efficiencies for much higher impact parameter values. It requires that a RSA muon is reconstructed in the top half of CMS and that it passes the following selection:

- $p_{T}>30 \mathrm{GeV} / \mathrm{c}$,

- $|\eta|<2$,

- $-40 \mathrm{~ns}<$ timeAtIpInOut $<-20 \mathrm{~ns}$,

- at least two valid hits in two DT or CSC stations,

- maximum transverse and longitudinal impact parameter errors of $10 \mathrm{~cm}$,

while the bottom muon is required to satisfy:

- $p_{T}>26 \mathrm{GeV} / \mathrm{c}$,

- $|\eta|<2$,

- at least 17 valid hits in the DT or CSC muon chambers, 
- at least three valid hits in two DT or CSC stations.

The efficiency is computed as the fraction of bottom muons found when a top muon is also found and is shown in Figure 6.11.

The results of these two measurements do not need to yield the same absolute value as they are integrated over different timing distributions. We can select the timing for the top muons, while for the silicon tracker tracks the timing is constrained by the charge integration time of the detector and no direct measurement of the track arrival time is available. Additionally, because the resolution on the impact parameters from silicon tracker tracks and from muon chamber tracks is significantly different, the effective ranges analyzed are affected in different ways by bin migration effects. The aim of these measurements is to provide a comparison between data and simulation, so the conditions need only to be consistent within a given method.
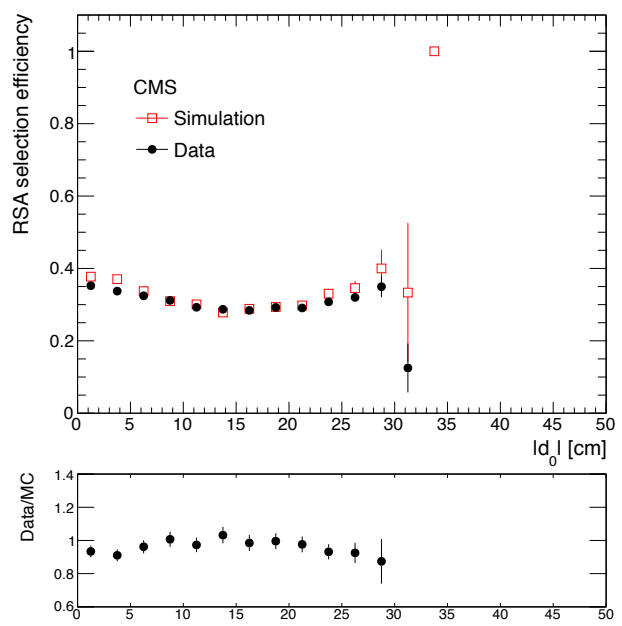

Figure 6.10.: RSA muon reconstruction and selection efficiency measured by requiring the presence of a reconstructed track in the silicon tracker as a function of $\left|d_{0}\right|$.

To estimate the systematic uncertainty associated to the simulation of the track reconstruction and selection efficiency for the dimuon candidates, we take into account the $\left|d_{0}\right|$ distributions of each muon in signal MC samples. If $\left\langle\epsilon_{\text {Data }}\right\rangle$ and $\left\langle\epsilon_{M C}\right\rangle$ are 
the weighted mean efficiencies to reconstruct both muon tracks in $X \rightarrow \mu^{+} \mu^{-}$decays, then the ratio of the two can be written as:

$$
\frac{\left\langle\epsilon_{\text {Data }}\right\rangle}{\left\langle\epsilon_{M C}\right\rangle}=\frac{\sum_{i} \sum_{j} g_{M C}(i, j) \epsilon_{\text {Data }}(i) \epsilon_{\text {Data }}(j)}{\sum_{i} \sum_{j} g_{M C}(i, j) \epsilon_{M C}(i) \epsilon_{M C}(j)}
$$

where:

- $i$ and $j$ are bins in $\left|d_{0}\right|$ distributions of the two muons respectively as shown in Figure 6.12.

- $g_{M C}(i, j)$ is the number of generated signal decays in which the two muons have $\left|d_{0}\right|$ in bin $(i, j)$ in a given MC sample.

- $\epsilon_{\text {Data }}(i)$ and $\epsilon_{M C}(i)$ are the efficiencies to reconstruct a single muon with $\left|d_{0}\right|$ in bin $i$ in data and MC, as given in Figure 6.11.

The ratios for all signal MC samples are shown in Figure 6.13. We conclude from this figure that a $18 \%$ systematic uncertainty per candidate would cover all considered signals.
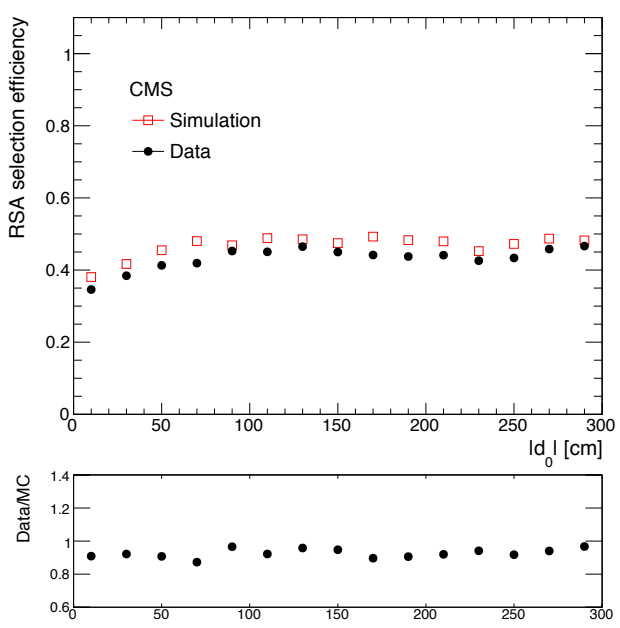

Figure 6.11.: RSA muon reconstruction and selection efficiency measured using only the muon chambers as a function of $\left|d_{0}\right|$ for $\left|z_{0}\right|<50 \mathrm{~cm}$. 

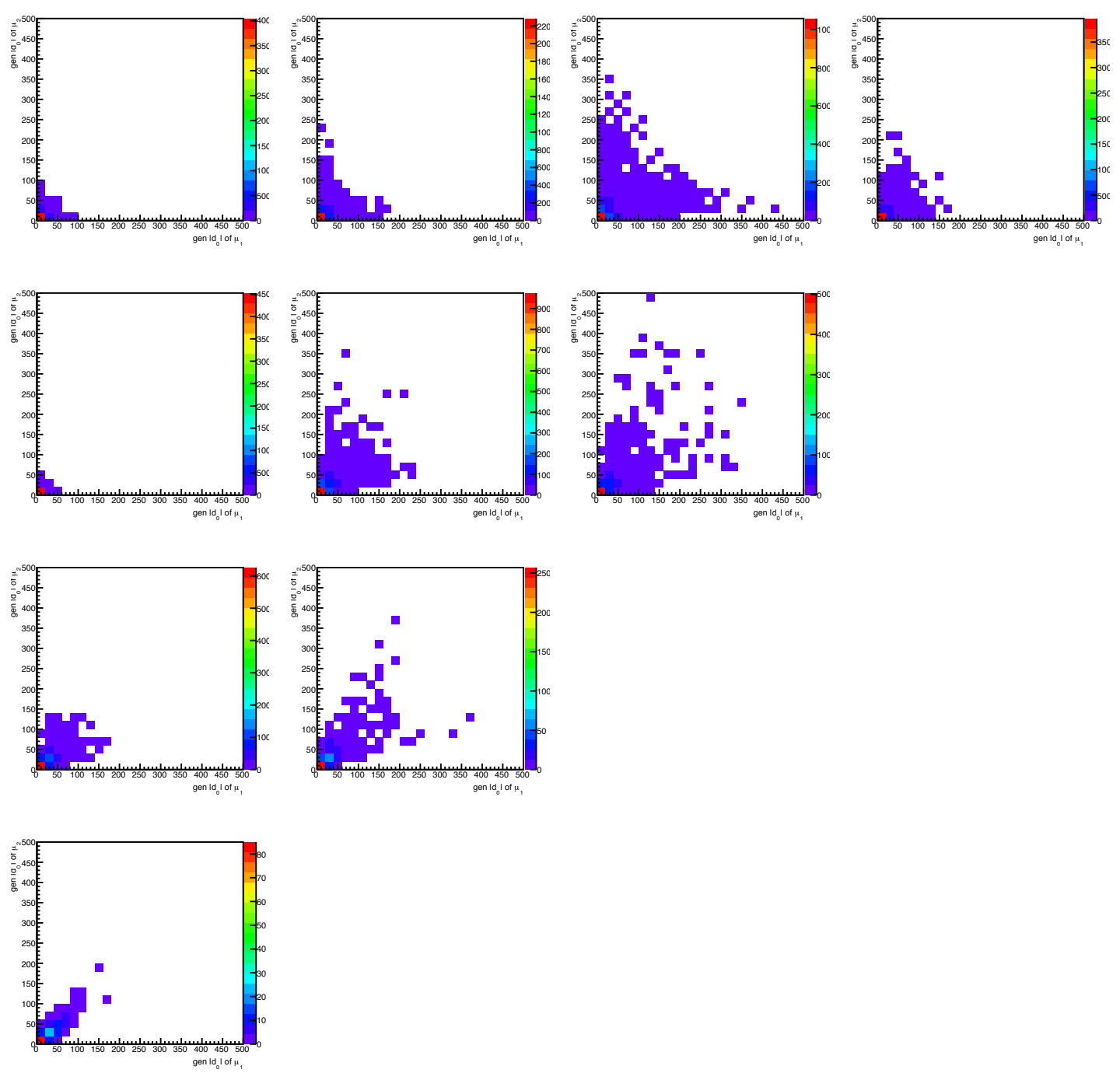

Figure 6.12.: $\left|d_{0}\right|$ distributions of both muons from the decay of the same LL particle in Monte Carlo signal samples. From left to right, $M_{X}=20,50,150$, and $350 \mathrm{GeV} / c^{2}$ and from top to bottom, $M_{H}=1000,400,200$, and $125 \mathrm{GeV} / c^{2}$. The generated $c \tau$ of each sample can be found from Figure 6.13. 


\subsection{Trigger efficiency measurement}

The systematic uncertainty assigned to the trigger efficiency to select the events analyzed is simply the discrepancy associated with that measurement between data and the simulation. The trigger efficiency is measured via the Tag and Probe method which can be exploited provided that there is a mass resonance, such as Z boson, decaying to muon pairs.

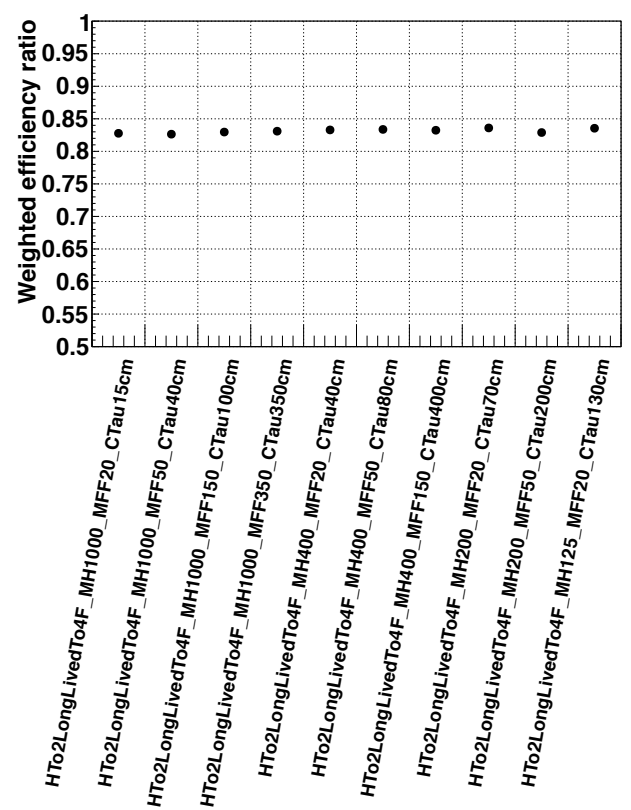

Figure 6.13.: Ratio of the weighted mean efficiencies convoluted with the signal MC distributions.

One of the muons, which comes from Z boson's decay, is labeled as tag that survives the tight selection criteria that ensure that it is very unlikely to be fake. The other muon, which is assumed to have no correlation with the tag muon, is called probe. A passing probe, satisfying selection cuts, is required to be matched to the 
trigger that selects the events for this analysis. Finally, one fits the tag-probe mass distribution to extract the number of $\mathrm{Z}$ candidates for failing and passing probes.

The analysis selects events collected by the trigger HLT_L2DoubleMu23_NoVertex_2Cha_Angle2p5, which will be denoted by Trigger A for convenience. This dimuon trigger requires:

- Two L2 muons with $p_{T}>23 \mathrm{GeV} / \mathrm{c}$ reconstructed with no vertex constraint.

- Each muon must have at least two DT or CSC muon stations with hits.

- The three dimensional angle between the two muons must be larger than 2.5 radians (cosine $>\sim-0.8)$.

Because of the angle requirement in the trigger, the collected dataset is unsuitable to apply the Tag and Probe method directly. Instead, we factor the trigger efficiency measurement into two parts. First, we use the events that are collected by another double muon trigger, HLT_L2DoubleMu23_NoVertex, henceforth called Trigger B, for the measurement. This trigger is identical to Trigger A except for the lack of the angle cut and of the requirement of a minimum number of muon stations with hits. Secondly, since the actual dimuon trigger is equivalent to Trigger B plus two additional cuts, we measure the efficiency of these additional cuts separately to get the overall efficiency of Trigger A used in this search. Note that Trigger B is prescaled in data by a factor of 20 , which is accounted for in the measurement.

To measure the efficiency of Trigger B, the tag is chosen from the global muon collection and is matched to an IsoMu24 single muon trigger object within $\Delta R<0.1$. Then, the following selection cuts are applied for the tag muon:

- $p_{T}>26 \mathrm{GeV} / \mathrm{c}$ and $|\eta|<2$

- Relative isolation (isolationR03.sumPt) $/ \mathrm{pt}<0.1$

- Number of tracker layers with measurement $\geq 6$

- $\left|d_{x y}\right|<30 \mathrm{~cm}$ and $\left|d_{z}\right|<30 \mathrm{~cm}$ 
The probe muons are required to be RSA muons used in the analysis. The conditions to be met for the probe muons are:

- $p_{T}>17 \mathrm{GeV} / \mathrm{c}$ and $|\eta|<2.4$

- Number of DT + CSC muon stations with valid hits $\geq 2$

- The probe is a passing probe if it is matched to the Trigger B object within $\Delta R<0.5$

In addition, the following two criteria are applied for the pair selection between the tag and the probe:

- $\Delta R>0.2$

- Cosine of the angle $>-0.79$

The efficiency for this measurement is input as an unknown parameter to the fitting. The trigger efficiency vs. $p_{T}$ of the probe muon for Trigger $\mathrm{B}$ is shown in Figure 6.14.

The efficiency of the additional cuts included in Trigger A relative to Trigger B is measured for the RSA muons. This efficiency is defined as the ratio of the number of events triggered by Trigger A, given that Trigger B is fired, to the number of events triggered by Trigger B only.

While keeping the selection requirements on RSA muons the same as described previously, the simultaneous fit is implemented to extract the ratio of the two trigger efficiencies as a function of the $p_{T}$ of one of the two RSA muons, which is chosen randomly, in the event triggered by both triggers. In Figure 6.15, the ratio of the Trigger A efficiency to the Trigger B efficiency vs. $p_{T}$ is presented. The efficiency to select two muons equals the square of the Trigger B efficiency multiplied by the ratio of Trigger A efficiency to Trigger B efficiency. The discrepancy between data and simulation is no larger than $10 \%$, which is taken as systematic uncertainty on the trigger efficiency measured for $\mathrm{Z}$ decays. The study is detailed in Appendix $\mathrm{C}$. 
Figure 6.16 shows that Trigger A efficiency is highly dependent on the lifetime, $c \tau$, of the decays in the effective range of the analysis. Note that the plots are obtained with the lifetime reweighting for the two signal $\mathrm{MC}$ samples with $M_{\mathrm{H}^{0}}=1000 \mathrm{GeV} / c^{2}$ and $M_{\mathrm{X}}=350 \mathrm{GeV} / c^{2} ; M_{\mathrm{H}^{0}}=400 \mathrm{GeV} / c^{2}$ and $M_{\mathrm{X}}=20 \mathrm{GeV} / c^{2}$.

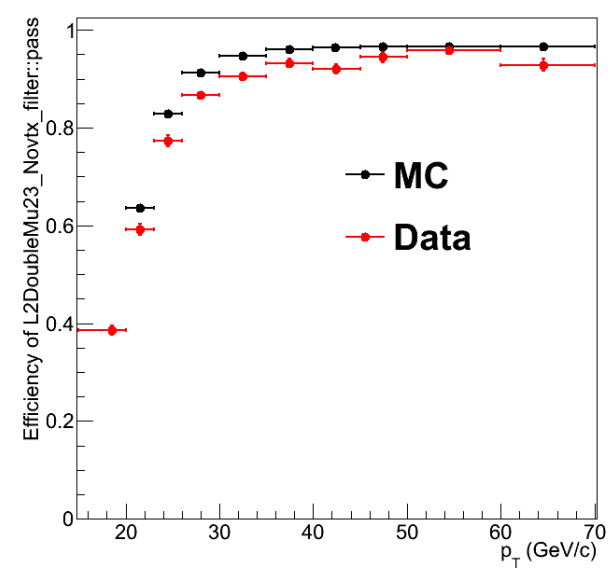

Figure 6.14.: Trigger efficiency of HLT_L2DoubleMu23_NoVertex vs. $p_{T}$ of the probe muon for both data and $\mathrm{Z} \rightarrow \mu \mu$ simulation, obtained with the Tag and Probe method.

The results discussed in Chapter 6.3 show that the agreement between RSA muon reconstruction and selection efficiencies in data and simulation is approximately independent of $\left|d_{0}\right|$. Since the algorithms used in the trigger muon reconstruction are similar to those used in the offline muon reconstruction, it is also reasonable to expect that the agreement between the trigger efficiencies does not strongly depend on $\left|d_{0}\right|$. Nevertheless, because we do not directly measure the trigger efficiency as a function of the decay length, we assign an additional systematic uncertainty by assuming that the difference in the trigger efficiency between data and simulation increases linearly as a function of the transverse decay length. The dimuon trigger efficiency to select the signal events is parameterized by a simple function of the transverse decay length, $L_{x y}$ : 


$$
F\left(L_{x y}\right)=0.9 \pm \frac{\beta L_{x y}}{250}
$$

indicating that the simulated trigger efficiency should be reduced by $10 \%$ for prompt events to overlap that of the data and that the additional variation by a factor of $\beta$ is added. $\beta$ is set to be $10 \%$ such that the trigger efficiency is varied by another $10 \%$ at 2.5 meters away from the interaction point, corresponding to the assumption that the discrepancy between data and simulation at that value is twice as big as for prompt decays.

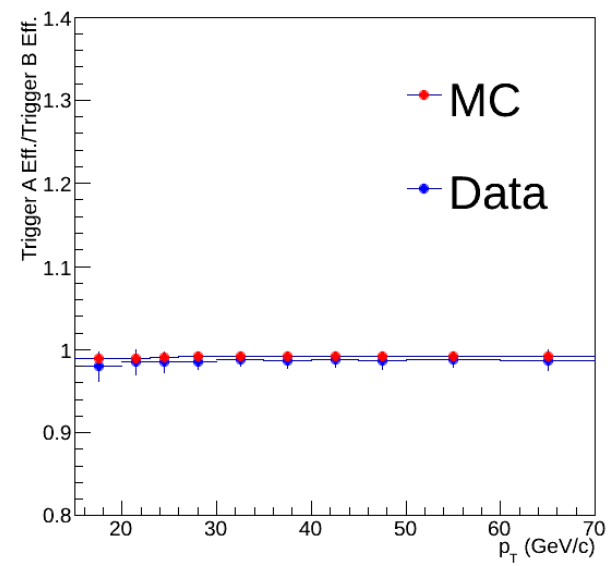

Figure 6.15.: Ratio of HLT_L2DoubleMu23_NoVertex_2Cha_Angle2p5 trigger efficiency to HLT_L2DoubleMu23_NoVertex trigger efficiency vs. $p_{T}$ for both data and $\mathrm{Z} \rightarrow \mu \mu$ simulation.

To conclude, the largest deviation in the signal selection efficiency is found to be not greater than $17 \%$, which we take as the relative systematic uncertainty on the trigger efficiency measurement in this analysis. 


\subsection{Effect of the $p_{\mathrm{T}}$ resolution}

We have studied the effect of the modest RSA muon $p_{\mathrm{T}}$ resolution on this analysis and conclude that no systematic uncertainty needs to be assigned. Appendix A discusses the study.
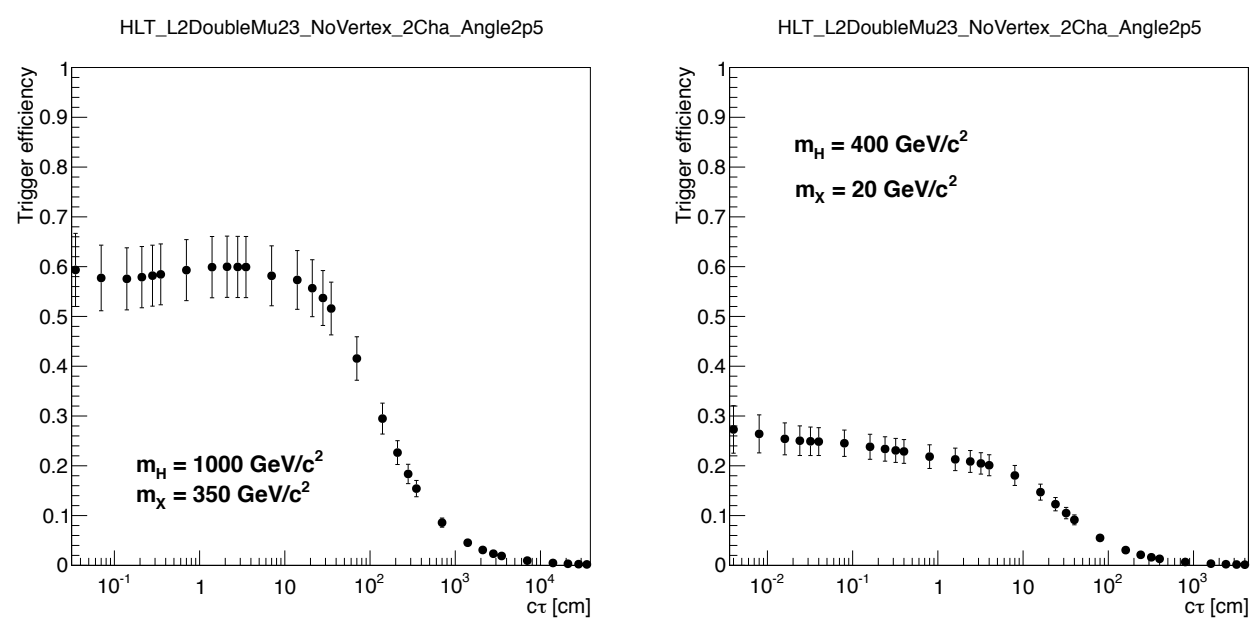

Figure 6.16.: Trigger efficiency of HLT_L2DoubleMu23_NoVertex_2Cha_Angle2p5 vs. generated decay length, $c \tau$, for the signal simulated events with $M_{\mathrm{H}^{0}}=1000 \mathrm{GeV} / c^{2}$ and $M_{\mathrm{X}}=350 \mathrm{GeV} / c^{2} ; M_{\mathrm{H}^{0}}=400 \mathrm{GeV} / c^{2}$ and $M_{\mathrm{X}}=20 \mathrm{GeV} / c^{2}$. Lifetime reweighting is applied to cover the full $c \tau$ range. The trigger efficiency starts to drop when $c \tau>10 \mathrm{~cm}$. 


\section{CHAPTER 7. ANALYSIS SENSITIVITY AND EXPECTED UPPER LIMITS}

The expected upper limits on the signal production mechanism based on a particular model with various mass points and lifetime values are set with $95 \%$ confidence level (CL). The computation is performed via the statistics software package developed by the CMS Higgs Group [19], which applies the Bayesian method established in Ref. [20]. The limits are derived by comparing the number of events $N_{S}$ expected in the signal region with the number of events that the signal plus background hypothesis predicts.

The systematic uncertainties on the signal selection efficiency given in Chapter 6 are introduced in the limit calculation as nuisance parameters with log-normal prior distributions. The expected number of background events in the signal region, $\mu_{B}$, is taken as an additional nuisance parameter, which depends on the number of observed events in the control region, $N_{C}$, and therefore in the signal region. The probability

distribution of $\mu_{B}, p\left(\mu_{B}\right)$, is given by $p\left(\mu_{B}\right)=\frac{\mu_{B}^{N_{C}}}{N_{C} !} \exp \left(-\mu_{B}\right)$, as can be shown using Bayesian method assuming a flat prior in $\mu_{B}[20]$.

The expected number of signal events, $\mu_{S}$, takes the following form:

$$
\begin{aligned}
\mu_{S} & =\mathcal{L} \sigma\left[2 \mathrm{~B}(1-\mathrm{B}) \epsilon_{1}+\epsilon_{2} \mathrm{~B}^{2}\right](1-f) \\
& =2 \mathcal{L} \sigma \mathrm{B} \epsilon_{1}\left[1-\mathrm{B}\left(1-\frac{\epsilon_{2}}{2 \epsilon_{1}}\right)\right](1-f)
\end{aligned}
$$

where $\mathcal{L}$ is the integrated luminosity, $\epsilon_{(1,2)}$ are the signal efficiencies defined in Chapter $4.5, \sigma$ is the production cross section of $\mathrm{H}^{0} \rightarrow \mathrm{XX}$ and $\mathrm{B}$ is the branching fraction for the decay $\mathrm{X} \rightarrow \ell^{+} \ell^{-}$where each lepton refers to a muon. The parameter $f$ represents the ratio of the number of signal events falling into the control region as fake background to the number of signal events in the signal region. Although the effect is negligible for all signal samples used, the conservative value, $5 \%$, is set for this parameter. If the efficiencies, $\epsilon_{1}$ and $\epsilon_{2}$, are independent of each other, it can be 
shown that $\epsilon_{2}=1-\left(1-\epsilon_{1}\right)^{2}$. However, since the triggering of one LL decay can give rise to the triggering of two LL decays, the two efficiencies are correlated, that is, $\epsilon_{2} \geq 1-\left(1-\epsilon_{1}\right)^{2}$. To calculate the upper limits conservatively, the value of the expected number of signal events, $\mu_{S}$, can be minimized with $\epsilon_{2}=1-\left(1-\epsilon_{1}\right)^{2}$ in the equation (7.1),

$$
\mu_{S}=2 \mathcal{L} \sigma \mathrm{B} \epsilon_{1}\left[1-\mathrm{B} \epsilon_{1} / 2\right](1-f)
$$

In equation (7.2), the upper bounds on $\sigma \mathrm{B}$ depend on the branching ratio. Hence, the limits are derived for two extreme cases, $\mathrm{B} \epsilon_{1} \ll 1$ and $\mathrm{B}=1$.

The 95\% CL upper limits are calculated for all mass points of $\mathrm{H}^{0} \rightarrow \mathrm{XX}$ signal samples, listed in Table 3.2, as a function of $\mathrm{X}$ boson lifetime. The expected limits are illustrated in the plots in Figure 7.1. The analysis is least sensitive to the $M_{\mathrm{H}^{0}}=$ $125 \mathrm{GeV} / c^{2}$ case due to the low signal selection efficiencies in particular when $M_{\mathrm{X}}=$ $20 \mathrm{GeV} / c^{2}$. The limits improve as the decay lifetime increases, as expected, since the analysis has a negligible sensitivity for the transverse decay lengths less than $40 \mathrm{~cm}$. The green bands in these limit plots represent the $\pm 1 \sigma$ range of variation of the expected $95 \%$ CL limits.

The same lifetime reweighting procedure is applied as in Ref. [6] to obtain the upper limits on $\sigma \mathrm{B}$. The signal efficiency for a given lifetime is estimated by reweighting the lifetime distribution of the sample that has the closest generated lifetime value to the one for which the estimation is performed. If the uncertainty in the signal efficiency being recomputed is greater than $30 \%$, the upper bound for that lifetime is discarded.

\subsection{Comparison with the tracker-based analysis}

In this section we compare the results of this analysis with those of the trackerbased analysis [6]. 

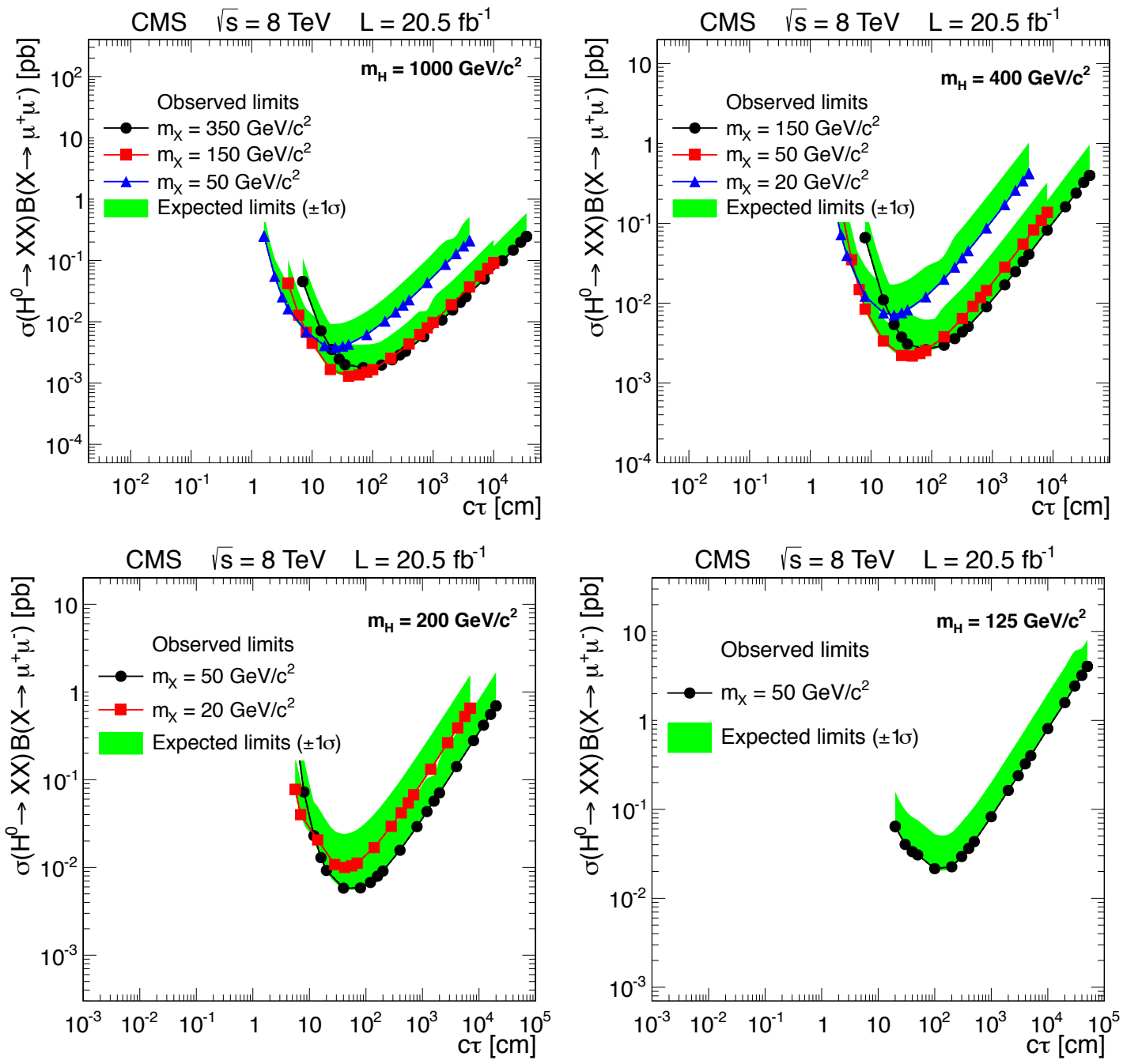

Figure 7.1.: 95\% CL upper limits on $\sigma\left(\mathrm{H}^{0} \rightarrow \mathrm{XX}\right) \mathrm{B}\left(\mathrm{X} \rightarrow \ell^{+} \ell^{-}\right)$for $M_{H^{0}}=$ $1000 \mathrm{GeV} / c^{2}, 400 \mathrm{GeV} / c^{2}, 200 \mathrm{GeV} / c^{2}$ and $125 \mathrm{GeV} / c^{2}$ with various X mass points. The limits derived for $\mathrm{B} \epsilon_{1} \ll 1$ are illustrated by the solid curves, whereas the dotted curves represent those for $\mathrm{B}=1$ (the dotted curves are difficult to discern due to the overlap with the solid curves). Green shaded bands show the $\pm 1 \sigma$ range of variation of the expected $95 \%$ CL limits. 
The signal efficiencies that the two analyses yield for the largest lifetime samples of $\mathrm{H}^{0} \rightarrow \mathrm{XX}$ simulated events are tabulated in Table 7.1. In addition, the signal efficiency ratio of the two analyses for various $\mathrm{H}^{0}$ and $\mathrm{X}$ masses as a function of $c \tau$ is shown in Figure 7.2. For signal events with smaller $c \tau$, the ratio is near zero because this analysis is not sensitive in that range by design. For signal events with larger $c \tau$, the efficiency of this analysis is similar to that of the tracker-based analysis.

Table 7.1.: Efficiencies of the signal MC samples to satisfy the full selection for the two analyses. The efficiencies for the events in which only one simulated LL particle decays to the muons, $\epsilon_{1}$, and for the ones where there are two generated LL particles decaying to the muon pairs, $\epsilon_{2}$, are shown separately.

\begin{tabular}{|c|c|c||c|c|c|c|}
\hline$M_{\mathrm{H}^{0}}$ & $M_{\mathrm{X}}$ & \multicolumn{2}{c||}{$c \tau$} & \multicolumn{2}{c|}{ Muon Chambers } & \multicolumn{2}{c|}{ Silicon Tracker } \\
\hline$\left(\mathrm{GeV} / c^{2}\right)$ & $\left(\mathrm{GeV} / c^{2}\right)$ & $(\mathrm{cm})$ & $\epsilon_{1}$ & $\epsilon_{2}$ & $\epsilon_{1}$ & $\epsilon_{2}$ \\
\hline 1000 & 350 & 350 & 0.023 & 0.044 & 0.03 & 0.058 \\
\hline 1000 & 150 & 100 & 0.045 & 0.076 & 0.05 & 0.1 \\
\hline 1000 & 50 & 40 & 0.018 & 0.022 & 0.043 & 0.093 \\
\hline 1000 & 20 & 15 & 0.0015 & 0.0017 & 0.0035 & 0.009 \\
\hline 400 & 150 & 400 & 0.015 & 0.04 & 0.018 & 0.039 \\
\hline 400 & 50 & 80 & 0.030 & 0.053 & 0.036 & 0.082 \\
\hline 400 & 20 & 40 & 0.0094 & 0.013 & 0.023 & 0.056 \\
\hline 200 & 50 & 200 & 0.0084 & 0.019 & 0.0094 & 0.026 \\
\hline 200 & 20 & 70 & 0.0068 & 0.012 & 0.016 & 0.039 \\
\hline 125 & 50 & 500 & 0.0018 & 0.0042 & 0.0013 & 0.0026 \\
\hline 125 & 20 & 130 & 0.0011 & 0.0027 & 0.0021 & 0.0054 \\
\hline
\end{tabular}



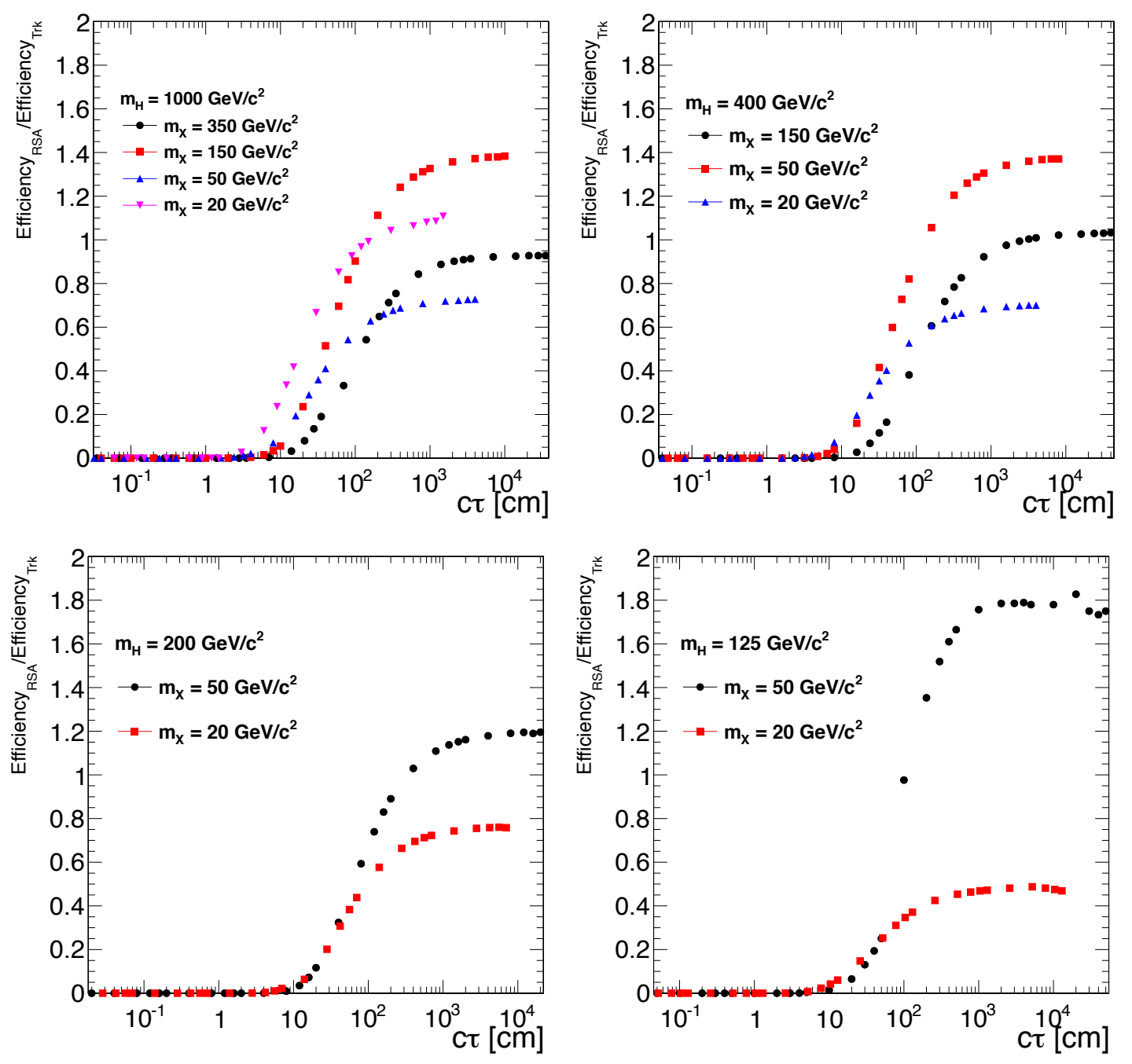

Figure 7.2.: Signal efficiency ratio of the two analyses $\epsilon_{R S A} / \epsilon_{t r k}$ for $\mathrm{H}^{0}$ mass values of $1000 \mathrm{GeV} / c^{2}, 400 \mathrm{GeV} / c^{2}, 200 \mathrm{GeV} / c^{2}$ and $125 \mathrm{GeV} / c^{2}$ with various $\mathrm{X}$ mass points. 


\subsection{Combined limits}

The analysis described in this document and the one based on the silicon tracker are orthogonal in the sense that there is no overlap in the events selected by the two analyses. The results are combined to yield the best possible exclusion limits. To produce the combination, we use the same framework that was used to compute the exclusion limits. The two analyses are treated as independent measurement channels of the same physics signal.

All the systematic uncertainties of the two channels are uncorrelated except for the integrated luminosity (fully correlated) and the trigger efficiency (partially correlated). For the trigger efficiency correlation we take the most conservative assumption when computing the combined expected limits. The tracker tracking efficiency systematics is also partially correlated because of the tracker track rejection cut applied in the muon chambers-based analysis. However, if this cut is removed, there is at most $2 \%$ overlap in the set of events satisfying the selection criteria of both analyses, meaning that only about $2 \%$ of the systematics is correlated. We consider this effect to be negligible and we assume no correlation for the systematic uncertainty on the tracker tracking efficiency.

The combined limits presented in this section are obtained with a preliminary and approximate method. We compute the observed limit using the tracker-based analysis results only. Since this limit depends uniquely on the properties of the Poisson distribution and it yields the value of three when zero events are observed (assuming this is the result of the unblinding), the result is accurate for both cases. We then scale the observed limit using the sum of the efficiencies of the two analyses. However, the expected limit band is not accurate as it does not take into account the background expectation and the systematic uncertainties of the muon chambersbased analysis. Nevertheless, the limit curves can be used to judge the improvement on the tracker-based analysis limits from the combination of the two results. 
The results of the combination are shown in Figure 7.3. The limits coincide with the ones from the tracker-based analysis for lower lifetime values, where the trackerbased analysis dominates the efficiency. For higher lifetime values the exclusions are significantly improved by the combination, up to a factor of two.

New combined limits will be computed using a more accurate statistical procedure and will replace the ones in this section. We do not expect the results to change qualitatively. 

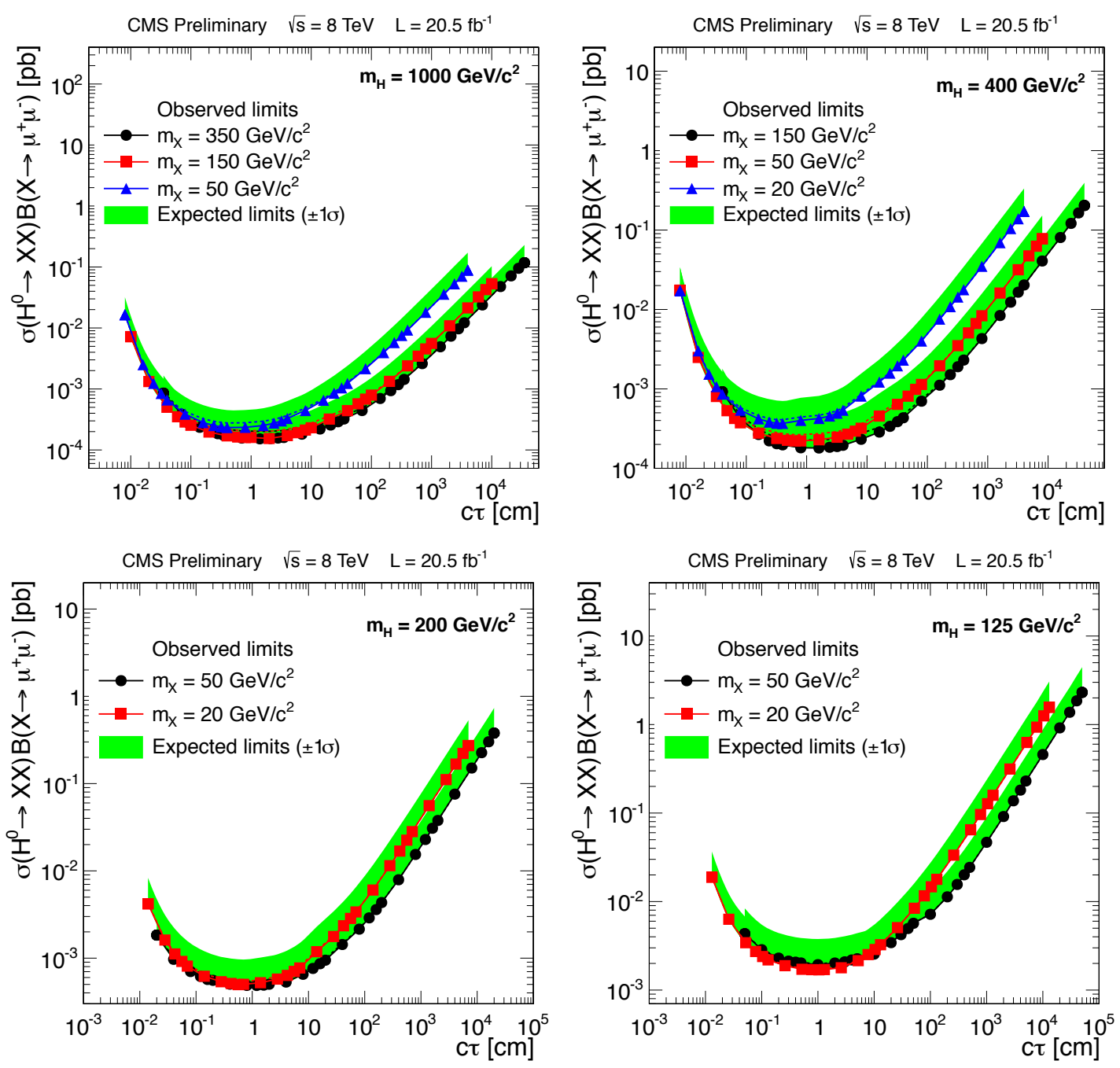

Figure 7.3.: Combined 95\% CL upper limits on $\sigma\left(\mathrm{H}^{0} \rightarrow \mathrm{XX}\right) \mathrm{B}\left(\mathrm{X} \rightarrow \ell^{+} \ell^{-}\right)$for all $\mathrm{H}^{0}$ mass values of $1000 \mathrm{GeV} / c^{2}, 400 \mathrm{GeV} / c^{2}, 200 \mathrm{GeV} / c^{2}$ and $125 \mathrm{GeV} / c^{2}$ with various $\mathrm{X}$ mass points. The limits derived for $\mathrm{B} \epsilon_{1} \ll 1$ are illustrated by the solid curves, whereas the dotted curves represent those for $\mathrm{B}=1$ (the dotted curves are difficult to discern due to the overlap with the solid curves). Green shaded bands show the $\pm 1 \sigma$ range of variation of the expected $95 \%$ CL limits. 


\section{CHAPTER 8. SUMMARY}

The first search at the LHC for long-lived particles decaying to dimuon final states using only the muon chambers has been performed on pp collision data taken by the CMS detector at $\sqrt{s}=8 \mathrm{TeV}$ in 2012. It extends the scope of a similar search for displaced dimuon signatures based on the silicon tracker. No background events are expected to pass the selection criteria of the analysis. Expected upper limits are computed for the model predicting a heavy scalar, with mass in the range 125 $1000 \mathrm{GeV} / c^{2}$, decaying to pairs of long-lived neutral particles, with masses in the range $20-350 \mathrm{GeV} / c^{2}$, which decay to dimuon pairs. The limits are typically in the range 1 - $20 \mathrm{fb}$, and can weaken to a few pb for the lowest masses and longest lifetimes, and are given for lifetimes in the range $1<c \tau<1000 \mathrm{~cm}$. The expected upper limits are comparable to, and in some cases improve on, those set by the tracker-based analysis, for longer lifetimes. Given that the two analyses are fully orthogonal, combined upper limits are presented which provide the most stringent limits for this kind of search in the dimuon channel so far. 


\section{REFERENCES}




\section{REFERENCES}

[1] JoAnne L. Hewett, Ben Lillie, Manuel Masip, and Thomas G. Rizzo. Signatures of long-lived gluinos in split supersymmetry. JHEP, 09:070, 2004.

[2] R. Barbier, C. Berat, M. Besancon, M. Chemtob, A. Deandrea, et al. R-parity violating supersymmetry. Phys. Rept., 420:1, 2005.

[3] Tao Han, Zongguo Si, Kathryn M. Zurek, and Matthew J. Strassler. Phenomenology of hidden valleys at hadron colliders. JHEP, 07:008, 2008.

[4] Lorenzo Basso, Alexander Belyaev, Stefano Moretti, and Claire H. ShepherdThemistocleous. Phenomenology of the minimal B-L extension of the Standard model: Z' and neutrinos. Phys. Rev. D, 80:055030, 2009.

[5] Matthew J. Strassler and Kathryn M. Zurek. Discovering the Higgs through highly-displaced vertices. Phys. Lett. B, 661:263, 2008.

[6] CMS Collaboration. Search for long-lived particles decaying to final states that include dileptons. 2014.

[7] V.M. Abazov et al. Search for neutral, long-lived particles decaying into two muons in $p \bar{p}$ collisions at $\sqrt{s}=1.96-\mathrm{TeV}$. Phys. Rev. Lett., 97:161802, 2006.

[8] V.M. Abazov et al. Search for long-lived particles decaying into electron or photon pairs with the D0 detector. Phys. Rev. Lett., 101:111802, 2008.

[9] Georges Aad et al. Search for a light Higgs boson decaying to long-lived weaklyinteracting particles in proton-proton collisions at $\operatorname{sqrt}(\mathrm{s})=7 \mathrm{TeV}$ with the ATLAS detector. Phys. Rev. Lett., 108:251801, 2012.

[10] Georges Aad et al. Search for displaced vertices arising from decays of new heavy particles in $7 \mathrm{TeV}$ pp collisions at ATLAS. Phys. Lett. B, 707:478, 2012.

[11] R. Adolphi et al. The CMS experiment at the CERN LHC. JINST, 03:S08004, 2008.

[12] The CMS collaboration. Performance of CMS muon reconstruction in pp collision events at $\sqrt{s}=7 \mathrm{TeV}$. Journal of Instrumentation, 7(10):P10002, 2012.

[13] S. Mrenna T. Sjöstrand and P. Z. Skands. PYTHIA 6.4 Physics and Manual. JHEP, 05:576, 2006.

[14] Stefano Frixione, Paolo Nason, and Carlo Oleari. Matching NLO QCD computations with Parton Shower simulations: the POWHEG method. JHEP, 0711:070, 2007. 
[15] S. Agostinelli et al. GEANT4: A simulation toolkit. Nucl. Instrum. Meth. A, 506:250, 2003.

[16] CMS Collaboration. CMS luminosity based on pixel cluster counting - Summer 2013 update. CMS Physics Analysis Summary CMS-PAS-LUM-13-001, 2013.

[17] Cms reweighting procedure for pile-up. https://twiki.cern.ch/twiki/bin/ view/CMS/PileupReweighting.

[18] Cms estimating systematic errors due to pile-up modeling. https://twiki. cern.ch/twiki/bin/viewauth/CMS/PileupSystematicErrors.

[19] Roostats-based statistics tools for higgs pag. https://twiki.cern.ch/twiki/ bin/view/CMS/SWGuideHiggsAnalysisCombinedLimit.

[20] ATLAS Collaboration, CMS Collaboration, LHC Higgs Combination Group. Procedure for the LHC Higgs boson search combination in Summer 2011. Public Note ATL-PHYS-PUB-2011-011, ATL-COM-PHYS-2011-818, CMS-NOTE2011-005, 2011.

[21] T. Speer, K. Prokofiev, R. Fruehwirth, W. Waltenberger, and P. Vanlaer. Vertex fitting in the CMS Tracker. CMS Internal Note CMS-NOTE-2006/032, 2006. 
APPENDICES 


\section{APPENDIX A. PERFORMANCE OF REFITTEDSTANDALONE MUONS}

The motivation for making use of the muons reconstructed using only the muon system arises from the fact that the LL neutral particles might also decay outside the reconstruction range of the silicon tracker. Thus, the hits in the muon chambers may give us hints for such particles when they decay to muon pairs in the reconstruction range of the muon system. In order to perform a systematic study on the reconstruction performance of the muon system for the displaced muons, the simulation samples containing the events with a pair of muons that have fixed $p_{T}$, transverse momentum, and several $d_{0}$, transverse impact parameter, are generated via the package ParticleGun. In this section, distributions of the track parameters are compared for two muon collections, namely standAlone (SA) and refittedStandAlone (RSA) muons to examine which track parameters can provide a good separation between prompt and displaced muons. Finally, the reconstruction efficiencies of the muon chambers are tabulated for the prompt and displaced muons with various impact parameters.

It is our crucial task to understand the reconstruction quality of the muons reconstructed in the muon chambers and investigate the muon track parameters and their resolutions. The simple design of the simulation events that are studied is as follows: Each event contains two back-to-back muons perpendicular to the x-axis of the transverse plane of the CMS detector. We generated 5 simulation samples in which there are 7000 events with $p_{T}$ of $100 \mathrm{GeV} / c$ and $d_{0}$ of $0,1,3,4$ and $5 \mathrm{~m}$, respectively. Additionally, we generated another sample, which consists of one million events that are uniform in both $p_{T}(0-1000 \mathrm{GeV} / c)$ and $d_{0}(0-100 \mathrm{~cm})$ to study the dependence of the reconstruction efficiency on the $p_{T}$ and $d_{0}$. 


\section{A.1 Track parameters of RSA and SA muons}

In this part, the distributions of various parameters of the prompt and displaced muons with $d_{0}=1 \mathrm{~m}$ are shown for the comparison between the SA and RSA muons. Note that RSA muons are the ones used in the analysis and they are derived from the SA muon class by removing the bias towards the beam spot. In Figure A.1 and Figure A.2, $p_{T}$ resolution vs. the number of valid hits per track plots are illustrated. It appears that both collections do not have a high performance in the $p_{T}$ resolution. Nevertheless, Figure A.2 suggests that RSA muons perform better for displaced muons, as expected.

In Figure A.3 and Figure A.4, $d_{0}$ resolution vs. the number of valid hits plots are presented. The RSA muons have a good $d_{0}$ resolution for displaced muons, although the $p_{T}$ measurement can be inaccurate. Figure A.5 and Figure A.6 illustrate $p_{T}$ resolution vs. $p_{T}$ significance graphs indicating that the transverse momentum resolution becomes slightly better as the transverse momentum significance increases for the prompt SA muons. Figure A.7 and Figure A.8 show $d_{0}$ resolution vs. $d_{0}$ significance plots, which give us an idea about how to distinguish the displaced muons from the prompt ones. The prompt RSA muons tend to have very low absolute $d_{0}$ significance values. On the other hand, the displaced ones are less likely to have low $d_{0}$ significance. Hence, by placing an appropriate cut, one can reject a significant amount of prompt events.

Figure A.9 and Figure A.10 reinforce the argument that the displaced muons have larger absolute $d_{0}$ significance values. No obvious relation between the $p_{T}$ significance and the number of valid hits is observed in Figure A.11 and Figure A.12. Finally, Figure A.13 and Figure A.14 suggest that the error in the measurement of $d_{0}$ for the displaced SA muons is slightly larger than that of the displaced RSA muons.

In conclusion, the cut on the $d_{0}$ significance can efficiently separate the displaced muons from the prompt ones. Displaced RSA muons have a good $d_{0}$ resolution yet poor $p_{T}$ resolution. 


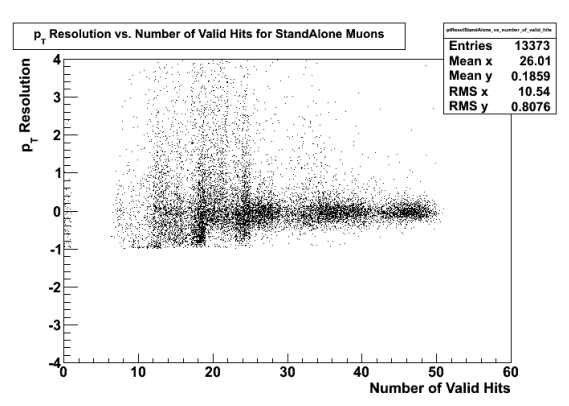

(a) SA Muons

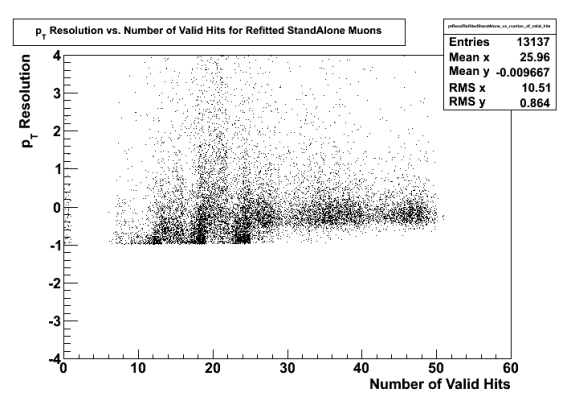

(b) RSA Muons

Figure A.1.: $p_{T}$ resolution vs. the number of valid hits for the prompt muons

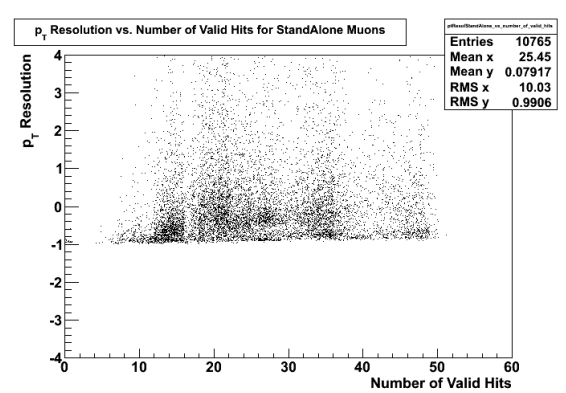

(a) SA Muons

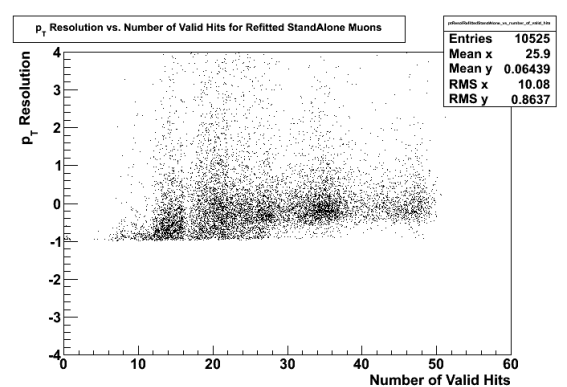

(b) RSA Muons

Figure A.2.: $p_{T}$ resolution vs. the number of valid hits for the displaced muons with $d_{0}=1 \mathrm{~m}$

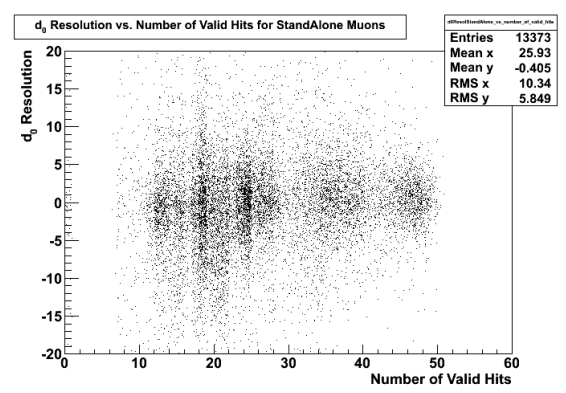

(a) SA Muons

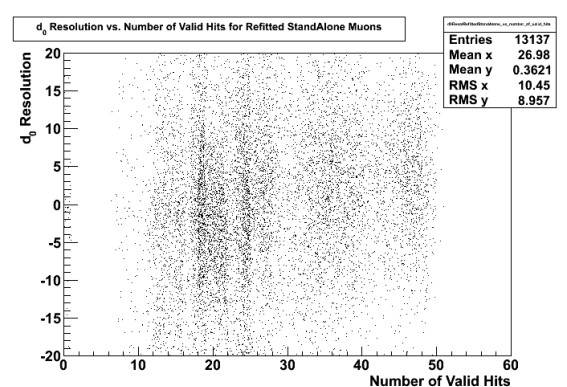

(b) RSA Muons

Figure A.3.: $d_{0}$ resolution vs. the number of valid hits for the prompt muons 


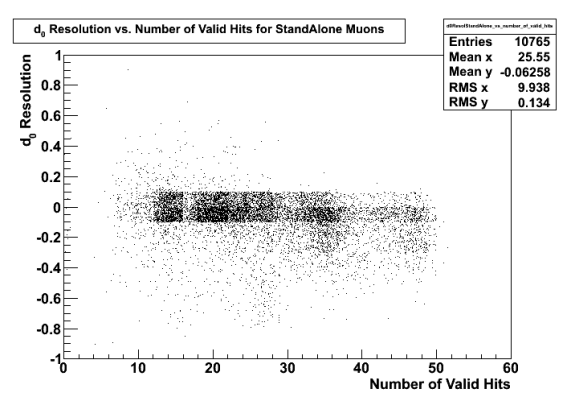

(a) SA Muons

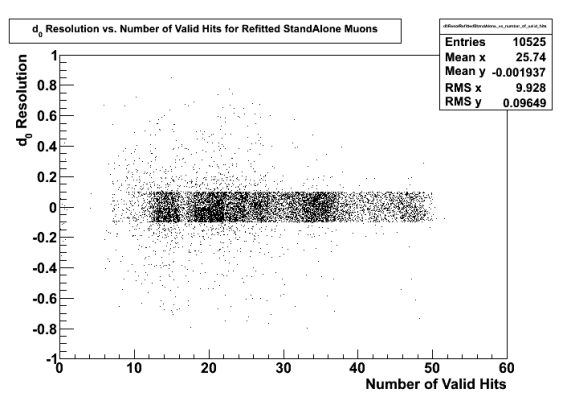

(b) RSA Muons

Figure A.4.: $d_{0}$ resolution vs. the number of valid hits for the displaced muons with $d_{0}=1 \mathrm{~m}$

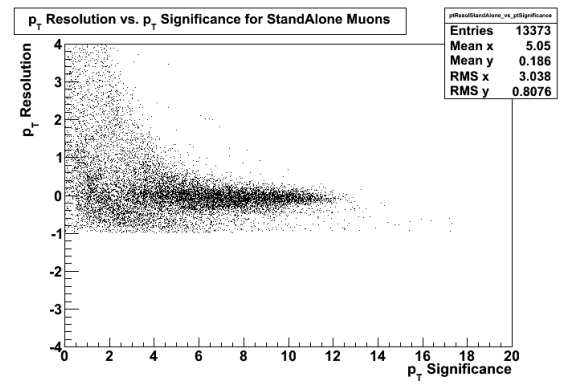

(a) SA Muons

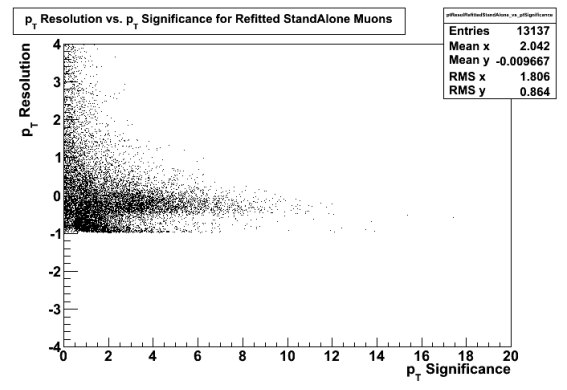

(b) RSA Muons

Figure A.5.: $p_{T}$ resolution vs. $p_{T}$ significance for the prompt muons

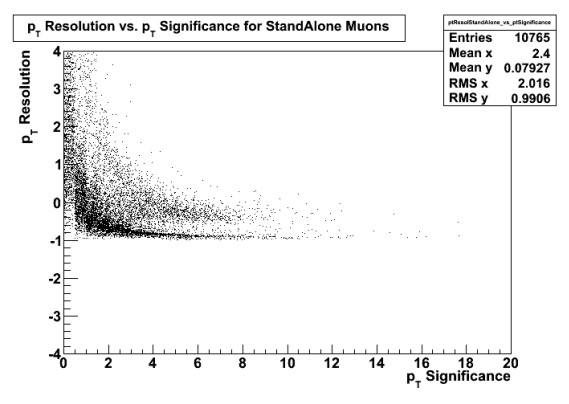

(a) SA Muons

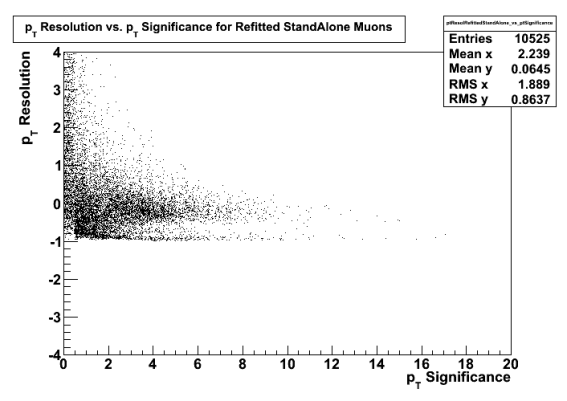

(b) RSA Muons

Figure A.6.: $p_{T}$ resolution vs. $p_{T}$ significance for the displaced muons with $d_{0}=1 \mathrm{~m}$ 


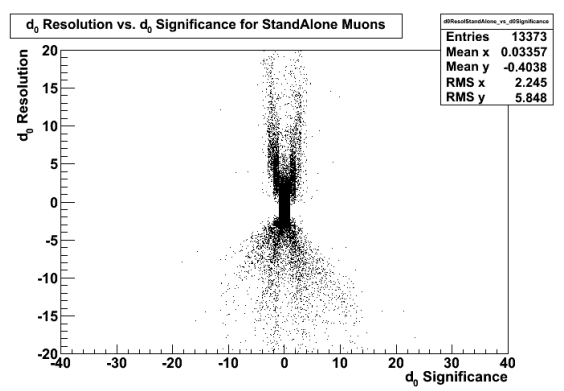

(a) SA Muons

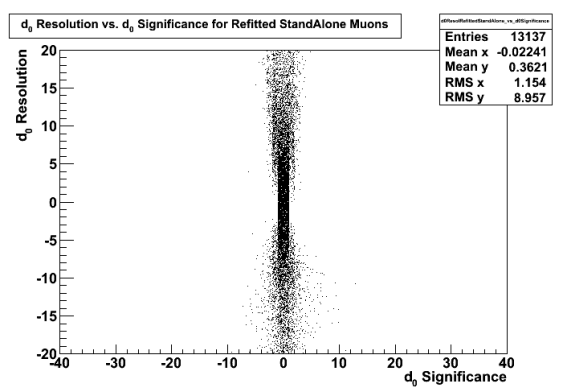

(b) RSA Muons

Figure A.7.: $d_{0}$ resolution vs. $d_{0}$ significance for the prompt muons

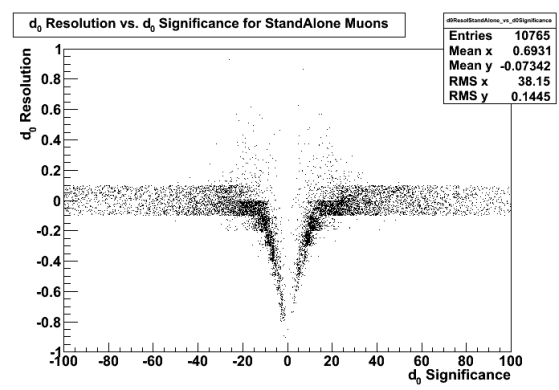

(a) SA Muons

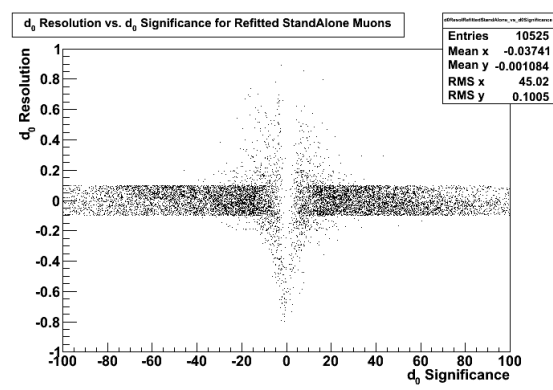

(b) RSA Muons

Figure A.8.: $d_{0}$ resolution vs. $d_{0}$ significance for the displaced muons with $d_{0}=1 \mathrm{~m}$

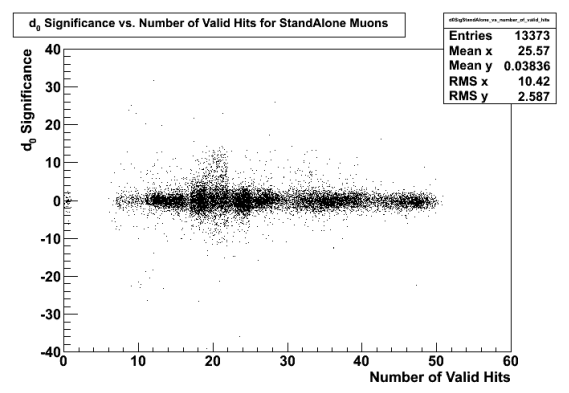

(a) SA Muons

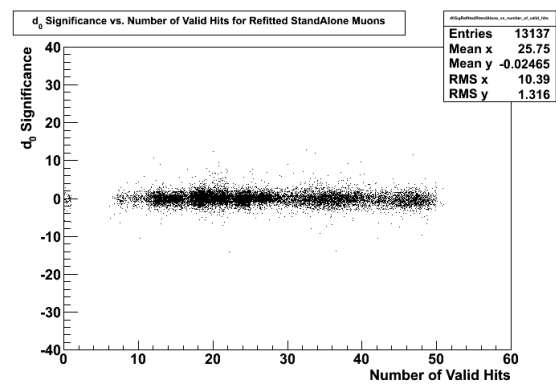

(b) RSA Muons

Figure A.9.: $d_{0}$ significance vs. the number of valid hits for the prompt muons 


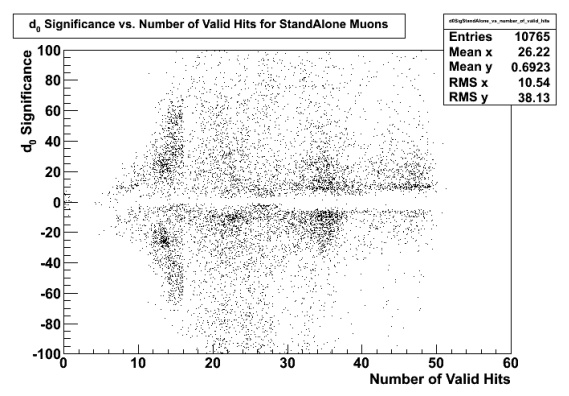

(a) SA Muons

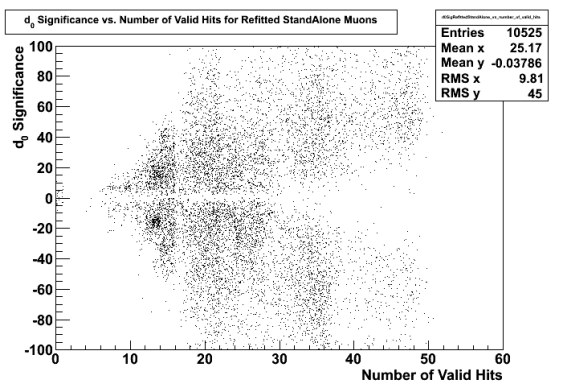

(b) RSA Muons

Figure A.10.: $d_{0}$ significance vs. the number of valid hits for the displaced muons with $d_{0}=1 m$

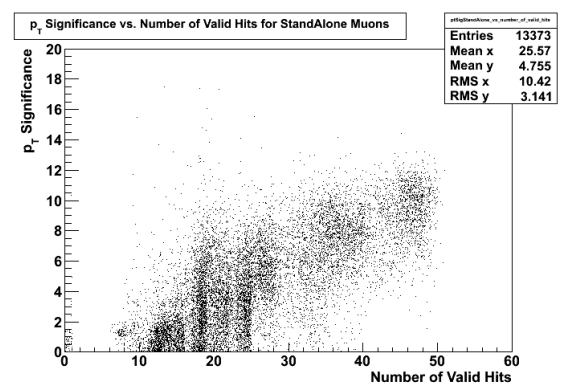

(a) SA Muons

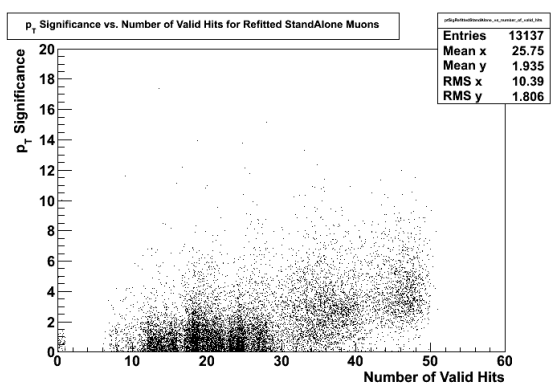

(b) RSA Muons

Figure A.11.: $p_{T}$ significance vs. the number of valid hits for the prompt muons

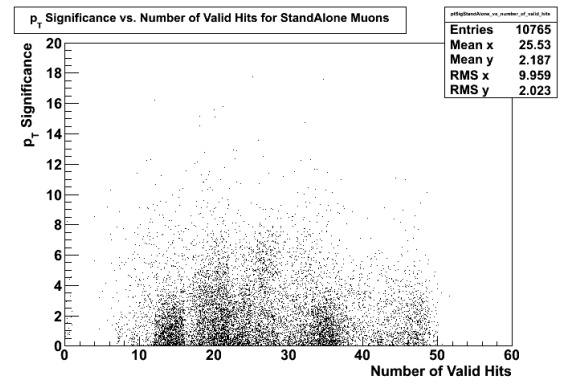

(a) SA Muons

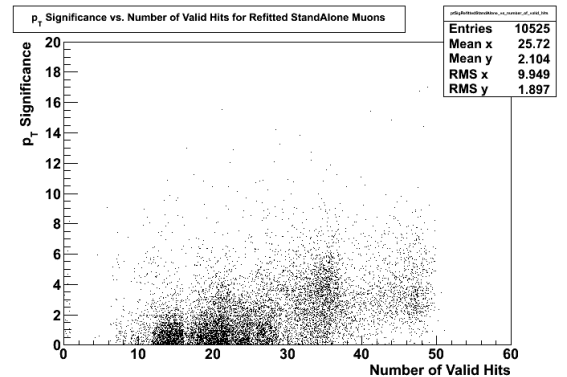

(b) RSA Muons

Figure A.12.: $p_{T}$ significance vs. the number of valid hits for the displaced muons with $d_{0}=1 m$ 


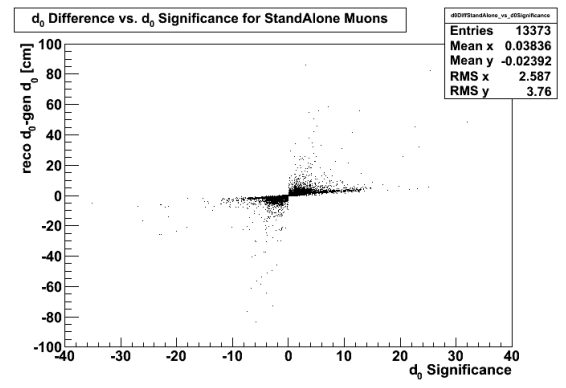

(a) SA Muons

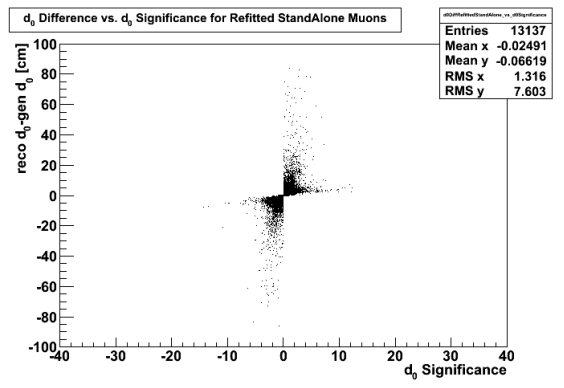

(b) RSA Muons

Figure A.13.: The difference between reco $d_{0}$ and gen $d_{0}$ vs. $d_{0}$ significance for the prompt muons

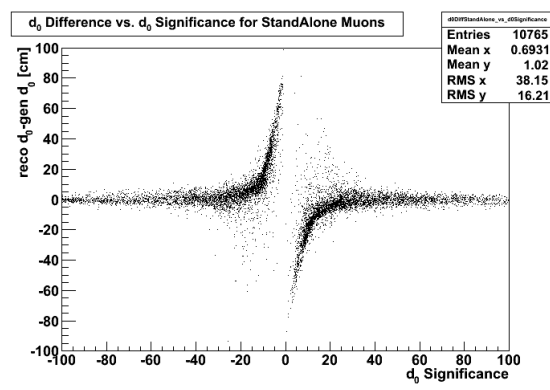

(a) SA Muons

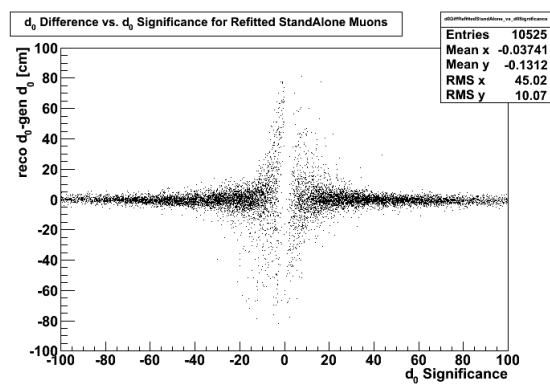

(b) RSA Muons

Figure A.14.: The difference between reco $d_{0}$ and gen $d_{0}$ vs. $d_{0}$ significance for the displaced muons with $d_{0}=1 \mathrm{~m}$ 


\section{A.2 Reconstruction efficiencies of prompt and displaced muons}

The reconstruction efficiencies of the prompt muons and of the muons with several fixed non-null $d_{0}$ values are compared. Table A.1 presents the reconstruction efficiencies of the muon system for muons with transverse impact parameters, $d_{0}$, of $0,1,3$, 4, 5 meters. Even $5 \mathrm{~m}$ away from the interaction point in the transverse plane, about a meter inside the muon system, the muon reconstruction efficiency is non-null, about $7 \%$. The reconstruction efficiency here is defined as the fraction of generated muons that are matched to the reconstructed ones. To show the reconstruction efficiency of the muon chambers as a function of $d_{0}$, three plots are made as illustrated in Figure A.15, Figure A.16 and Figure A.17 for the muons with $p_{T}$ of $10 \mathrm{GeV} / c, 100 \mathrm{GeV} / c$ and $1000 \mathrm{GeV} / c$, respectively. Note that the simulated sample that is uniform in both $p_{T}$ and $d_{0}$ is used to obtain these plots. They indicate that the muon reconstruction efficiency is usually above $90 \%$ up to $60 \mathrm{~cm}$. To conclude, the range of the analysis is not dominantly limited by the reconstruction efficiency of the muon chambers.

Table A.1.: Reconstruction efficiency of the muon chambers for the muons with several $d_{0}$

\begin{tabular}{ccc}
\hline \hline & SA Muons & RSA Muons \\
\hline Prompt Tracks & 0.9549 & 0.9384 \\
\hline Displaced Tracks with $d_{0}=1 m$ & 0.7685 & 0.7514 \\
\hline Displaced Tracks with $d_{0}=3 m$ & 0.3709 & 0.3639 \\
\hline Displaced Tracks with $d_{0}=4 m$ & 0.2378 & 0.2232 \\
\hline Displaced Tracks with $d_{0}=5 m$ & 0.0770 & 0.0695 \\
\hline
\end{tabular}




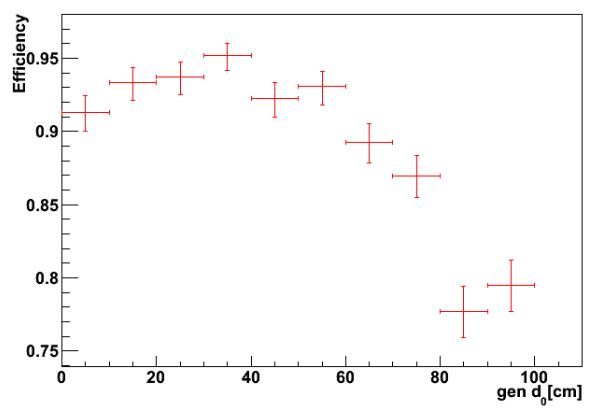

Figure A.15.: Reconstruction efficiency vs. $d_{0}$ for RSA muons with $p_{T}=10 \mathrm{GeV} / c$

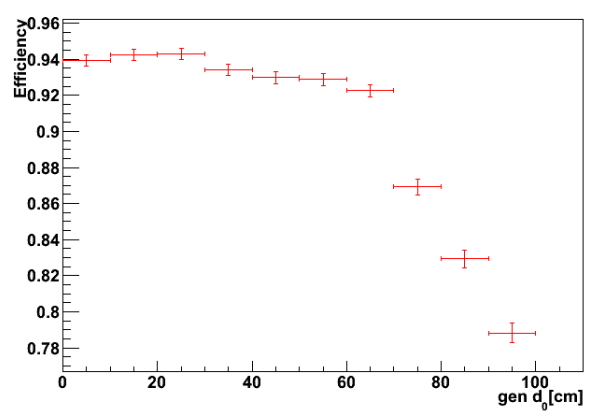

Figure A.16.: Reconstruction efficiency vs. $d_{0}$ for RSA muons with $p_{T}=100 \mathrm{GeV} / c$

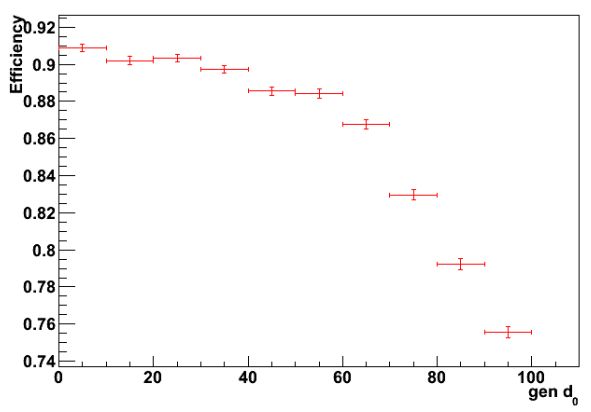

Figure A.17.: Reconstruction efficiency vs. $d_{0}$ for RSA muons with $p_{T}=1000 \mathrm{GeV} / c$ 


\section{APPENDIX B. MUON TIMING INFORMATION IN THE EVENTS REMOVED BY COSMIC REJECTION}

As an additional source of background in our analysis, we might have events in which at least one cosmic muon may enter and make fake dimuon combinations, which might be so highly displaced that they pass the lifetime selection, with other muons emanating from the pp collisions. Hence, the cosmic rejection cut, described in Chapter 4.3, is placed to avoid such cosmic contamination.

If a muon originating from cosmics hits the detector components randomly, it would most likely be unsynchronized with the time of the collisions. Even though the muon can still be reconstructed, the fit used to extract the timing information could fail. To examine the events vetoed by the cosmic rejection in detail, we check the timing information of the muons in those events. If a muon has the timing information reconstructed, it is labeled as in-time muon and out-of-time muon, if not.

It is checked that the efficiency of reconstructing muon timing is slightly above 99\% for the prompt data and the background simulation events. The same efficiency is around $93 \%$ for the signal simulation events. In other words, if a muon emerges from the collision, then, it is an in-time muon with great efficiency. On the other hand, if the events removed by the cosmic rejection are carefully investigated, more than $\approx 85 \%$ of the cases, we find at least one out-of-time muon in these events. Therefore, no decisive disagreement with the cosmic muon interference hypothesis is shown.

Another important finding is that there is a correlation between the muon timing reconstruction and the number of muon hits. To confirm it, we examine the valid muon hit distributions of in-time and out-of-time muons in the events removed by the cosmic rejection cut. Figure B.1 demonstrates the clear separation in the number of valid muon hits between the two. Thus, one could reasonably argue that out-of- 
time muons tend to have lower number of hits in the muon chambers and the timing reconstruction fails for the same reason.
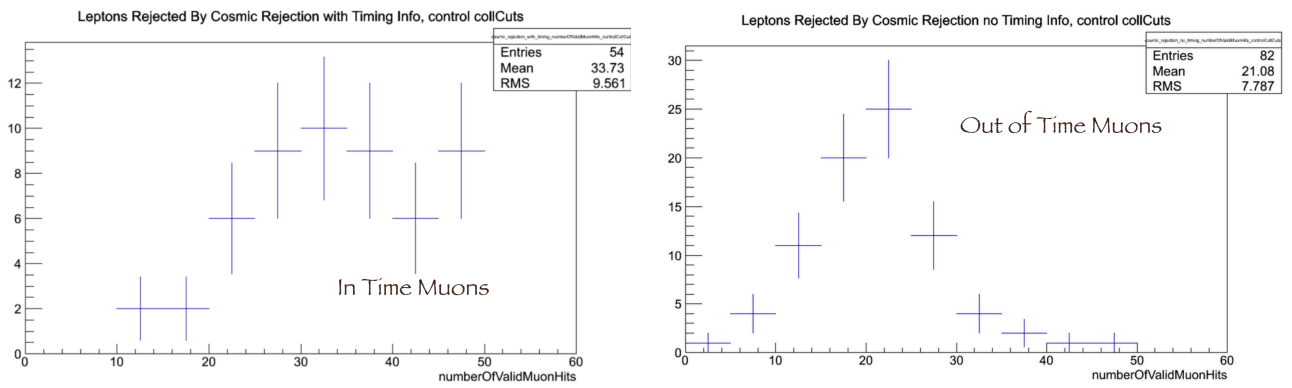

Figure B.1.: (Left) Distribution of the number of valid hits for the in-time muons in the events rejected by the cosmic rejection cut. (Right) Same distribution for the outof-time muons. There is an obvious shift between the two distributions, suggesting that out-of-time muons, in general, have lower number of hits in the muon chambers.

In Figure B.2, the distribution of the minimum number of valid hits of the muons passing the full selection is shown for one of the signal samples. After checking the same distribution in all signal simulated samples, it is concluded that if one requires at least 17 valid hits for each muon, the signal efficiencies would not be significantly affected, whereas the level of background in data would be slightly reduced. Therefore, this cut is included in the analysis selection as a further protection against the cosmic contamination. 


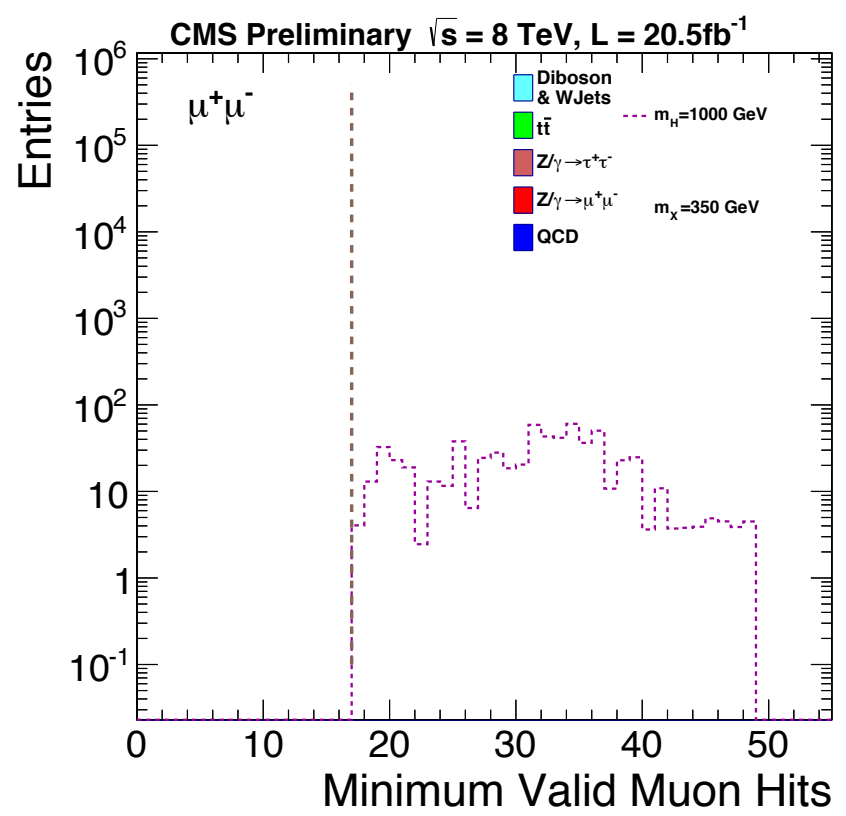

Figure B.2.: Distribution of the minimum valid muon hits of the two muons for the dimuon candidates passing the full selection except the cut on the minimum valid muon hits. This plot is obtained for the signal sample: $M_{\mathrm{H}^{0}}=1000 \mathrm{GeV} / c^{2}$ and $M_{\mathrm{X}}=350 \mathrm{GeV} / c^{2}$ with $c \tau=350 \mathrm{~cm}$ 


\section{APPENDIX C. MUON TRIGGER EFFICIENCY}

The systematic uncertainty assigned to the trigger efficiency to select the events analyzed is simply the discrepancy associated with that measurement between data and the simulation. The trigger efficiency is measured via the Tag and Probe method which can be exploited provided that there is a mass resonance, such as Z boson, decaying to muon pairs.

One of the muons, which comes from Z boson's decay, is labeled as tag that survives the tight selection criteria that ensure that it is very unlikely to be fake. The other muon, which is assumed to have no correlation with the tag muon, is called probe. A passing probe, satisfying selection cuts, is required to be matched to the trigger that selects the events for this analysis. Finally, one fits the tag-probe mass distribution to extract the number of $\mathrm{Z}$ candidates for failing and passing probes.

The analysis selects events the collected by the trigger, HLT_L2DoubleMu23_NoVertex_2Cha_Angle2p5. This dimuon trigger requires:

- Two L2 muons with $p_{T}>23 \mathrm{GeV} / \mathrm{c}$ reconstructed with no vertex constraint.

- Each muon must have at least two DT or CSC muon stations with any hits.

- The three dimensional angle between the two muons must be larger than 2.5 radians (cosine $>\sim-0.8)$.

Because of the angle requirement in the trigger, the collected dataset is unsuitable to apply the Tag and Probe method directly. Instead, we factor the trigger efficiency measurement into two parts. First, we use the events that are collected by another double muon trigger, HLT_L2DoubleMu23_NoVertex for the measurement. This trigger is identical to HLT_L2DoubleMu23_NoVertex_2Cha_Angle2p5 except for the lack of the angle cut and of the requirement of a minimum number of muon stations with hits. Secondly, since the actual dimuon trigger is equivalent to HLT_L2DoubleMu23_- 
NoVertex plus two additional cuts, we measure the efficiency of these additional cuts separately to get the overall efficiency of HLT_L2DoubleMu23_NoVertex_2Cha_Angle2p5 used in this search. Note that HLT_L2DoubleMu23_NoVertex is prescaled by a factor of 20 , which is accounted for the measurement.

The MC dataset used for this measurement is /DYJetsToLL_M-50_TuneZ2Star_8TeV-madgraph-tarball/Summer12_DR53XPU_S10_START53_V7Av1/AODSIM and all CMS datasets in this study are processed as 22Jan ReReco. In the part in which HLT_L2DoubleMu23_NoVertex trigger efficiency is measured, SingleMu CMS dataset is used. DoubleMu CMS dataset is used when the ratio of the two trigger efficiencies is computed. The official Tag and Probe package is exploited to measure the trigger efficiency of HLT_L2DoubleMu23_NoVertex. The package is run under CMSSW_5_3_7.

To measure the HLT_L2DoubleMu23_NoVertex trigger efficiency, the tag is chosen from the global muon collection matched to IsoMu24 single muon trigger object within $\Delta R<0.1$. Then, the following selection cuts are applied for the tag muon:

- $p_{T}>26 \mathrm{GeV} / \mathrm{c}$ and $|\eta|<2$

- Relative isolation (isolationR03.sumPt) $/ \mathrm{pt}<0.1$

- Number of tracker layers with measurement $\geq 6$

- $\left|d_{x y}\right|<30 \mathrm{~cm}$ and $\left|d_{z}\right|<30 \mathrm{~cm}$

The probe muons are required to be RSA muons used in the analysis. The conditions to be met for the probe muons are:

- $p_{T}>17 \mathrm{GeV} / \mathrm{c}$ and $|\eta|<2.4$

- Number of DT + CSC muon stations with valid hits $\geq 2$

- The probe is a passing probe if it is matched to the dimuon trigger, HLT_L2DoubleMu23_NoVertex, object within $\Delta R<0.5$

In addition, two generic criteria are applied for the pair selection between the tag and the probe:

- $\Delta R>0.2$ 
- Cosine of the angle $>-0.79$

The efficiency for this measurement is input as an unknown parameter to the simultaneous fitting. Once the fitting is done, the parameter is simply extracted and plotted. The trigger efficiency vs. $p_{T}$ graph for the dimuon trigger, HLT_L2DoubleMu23_NoVertex, is shown in Figure C.1. It can be deduced from the same graph that data-MC discrepancy is less than 5\%. Additionally, in order to check the validity of the efficiency result from the simultaneous fitting, a comparison with the efficiency results from counting method is performed for the simulation. Figure C.2 shows that the fit results agree with those from counting.

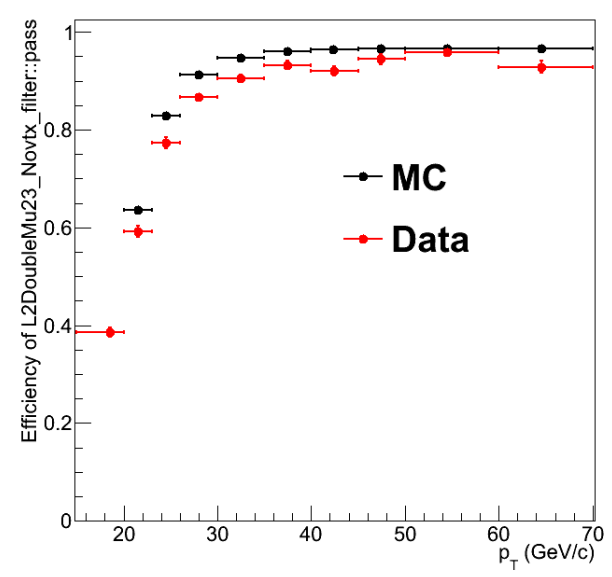

Figure C.1.: Trigger efficiency of HLT_L2DoubleMu23_NoVertex vs. $p_{T}$ of the probe muon for both data and $\mathrm{Z} \rightarrow \mu \mu$ simulation, obtained with the Tag and Probe method.

To demonstrate the accuracy of the fitting, one may look at Figure C.3 and Figure C.4 in which the tag-probe dimuon mass distributions are shown for each $p_{T}$ bin of the probe muon. Note that green line represents the dimuon mass distribution of the tags and the passing probes and blue line represents that of tags and all probes. The red curve is for the same distribution with the failing probes. It can be said that even though the fitting is not perfectly accurate, it is reasonable. Given that the 
offline muon $p_{T}$ threshold is $26 \mathrm{GeV} / c$ for the analysis, one should not consider the bins below that value for the accuracy check. Another important feature of the RSA muons seen from these figures is that $\mathrm{Z}$ mass peak location is different for each bin. It is mainly due to the low $p_{T}$ resolution of RSA muons. Besides, the fact that we have binning in $p_{T}$ makes the shift more obvious to discern. One may also suspect that some of the passing probes are fake. However, an additional study is performed with the MC sample from which MC Truth information is collected determining if the muons actually come from $\mathrm{Z}$ boson decay. In Figure C.5, red, blue and violet dots are the results from fitting, counting and $M C$ Truth fitting. The plot illustrates that $M C$ Truth fitting results are almost identical to the results in matching not-required case. The fitting and counting results are in agreement with each other as well.

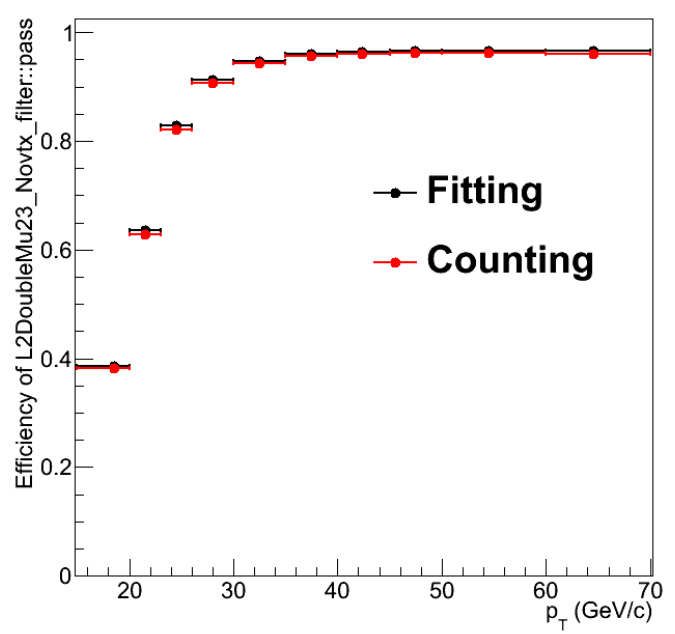

Figure C.2.: The plot compares HLT_L2DoubleMu23_NoVertex trigger efficiency results from the fitting, already presented in Figure 6.14 and with those from the counting method for the MC sample.

HLT_L2DoubleMu23_NoVertex trigger efficiency as a function of pseudorapidity, $|\eta|$, is also derived. Figure C.6 shows that the trigger efficiency is highest in the barrel region, which is expected. In addition, in order to have an idea of how the efficiency 
varies for data and the simulation in two dimensional plane of $p_{T}$ and $|\eta|$, the plots in Figure C.7 and Figure C.8 are prepared for a further inspection.

The efficiency of the additional cuts included in HLT_L2DoubleMu23_NoVertex_2Cha_Angle2p5 relative to HLT_L2DoubleMu23_NoVertex is measured for the RSA muons. This efficiency is defined as the ratio of the number of events triggered by HLT_L2DoubleMu23_NoVertex_2Cha_Angle2p5, given that HLT_L2DoubleMu23_NoVertex is fired, to the number of events triggered by HLT_L2DoubleMu23_NoVertex only.

While keeping the selection requirements on RSA muons same as described previously, the simultaneous fit is implemented to extract the ratio of the two trigger efficiencies as a function of the $p_{T}$ of one of the RSA, probe, muons in the event. In Figure C.9, the ratio of the new trigger, HLT_L2DoubleMu23_NoVertex_2Cha_Angle2p5, efficiency to the old trigger, HLT_L2DoubleMu23_NoVertex, efficiency vs. $p_{T}$ plot is presented. Note that the terms, new and old, are used for simplicity. As it can be inferred from the plot, the efficiency of the additional cuts is around $98 \%$ and the simulation-data discrepancy is less than $1 \%$. It is also advised to look at both Figure C.10 and Figure C.11 that justify that the fits are accurate enough for which Crystal Ball function is employed.

The efficiency to trigger two muons equals the square of HLT_L2DoubleMu23_NoVertex efficiency multiplied by the ratio of HLT_L2DoubleMu23_NoVertex_2Cha_Angle2p5 efficiency to HLT_L2DoubleMu23_NoVertex efficiency. The discrepancy between data and simulation is no larger than $10 \%$, which is taken as systematic uncertainty on the trigger efficiency measured for $\mathrm{Z}$ decays.

An accompanying cross check performed is to test if the cosine cut, $\cos \theta>-0.79$, applied to the muon pairs is reasonable. If it is correct, then one may expect to have a jump in HLT_L2DoubleMu23_NoVertex_2Cha_Angle2p5 trigger efficiency around this value. Figure C.12 verifies that the cut value is optimal. One may pursue the similar line of thought to check if $\Delta R>0.2$ cut is also a good choice. However, since the samples used are rich in the muon pairs from $\mathrm{Z}$ decays that are mostly back-to- 

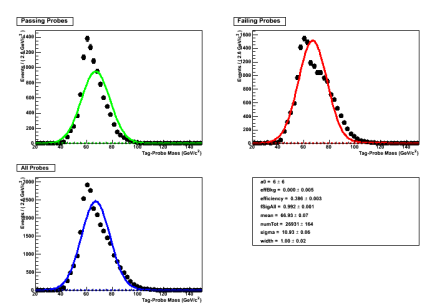

(a) $15-20 \mathrm{GeV}$
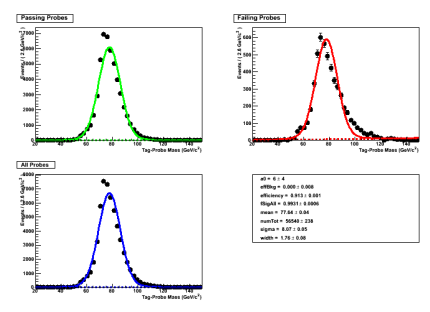

(d) $26-30 \mathrm{GeV}$
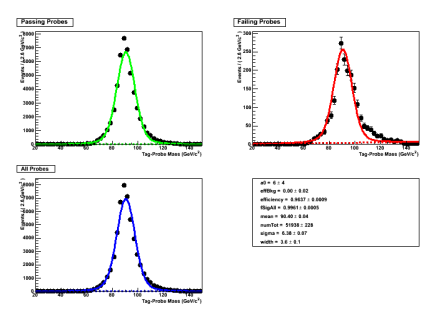

(g) $40-45 \mathrm{GeV}$

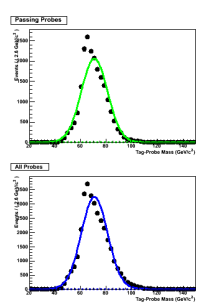

(b) $20-23 \mathrm{GeV}$

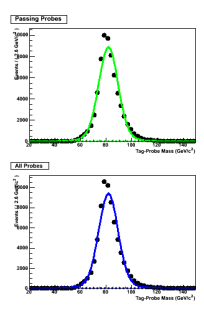

(e) $30-35 \mathrm{GeV}$

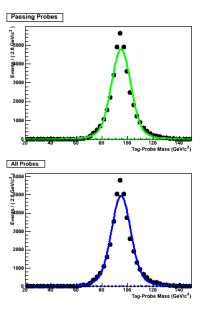

(h) $45-50 \mathrm{GeV}$
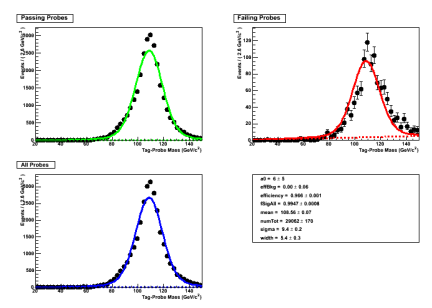

(j) $60-70 \mathrm{GeV}$

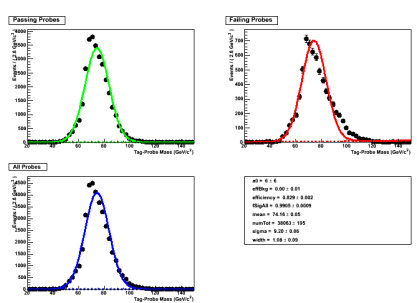

(c) $23-26 \mathrm{GeV}$
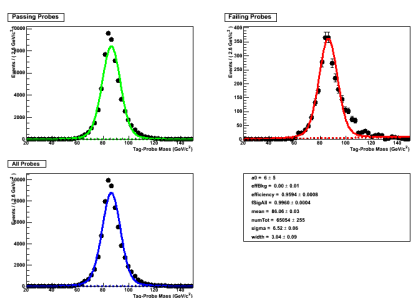

(f) $35-40 \mathrm{GeV}$
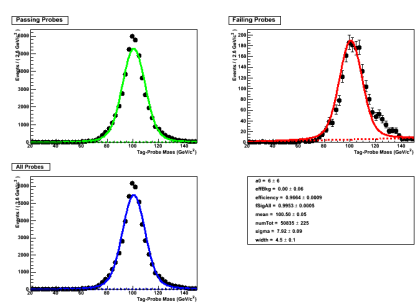

(i) $50-60 \mathrm{GeV}$

Figure C.3.: MC fitting results of tag-probe dimuon mass distributions for each $p_{T}$ bin with Tag and Probe applied for the trigger, HLT_L2DoubleMu23_NoVertex. Note that the range of each $p_{T}$ bin is indicated under each plot. 


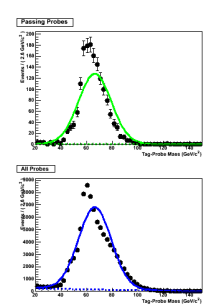

(a) $15-20 \mathrm{GeV}$

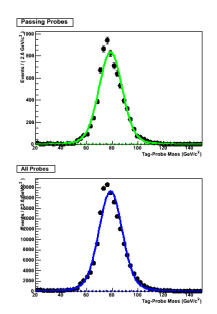

(d) $26-30 \mathrm{GeV}$

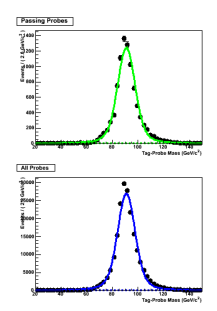

(g) $40-45 \mathrm{GeV}$

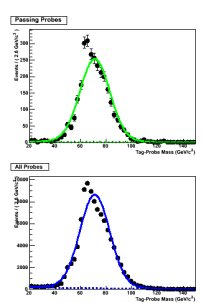

(b) $20-23 \mathrm{GeV}$

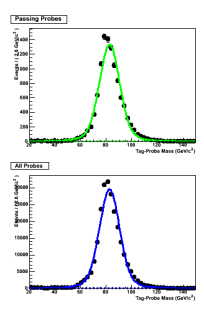

(e) $30-35 \mathrm{GeV}$

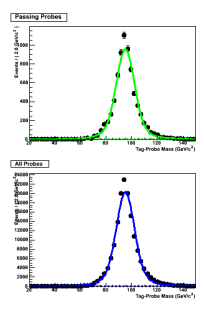

(h) $45-50 \mathrm{GeV}$
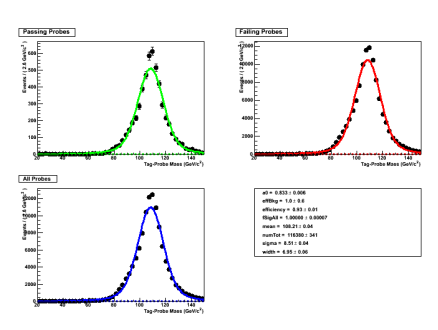

(j) $60-70 \mathrm{GeV}$

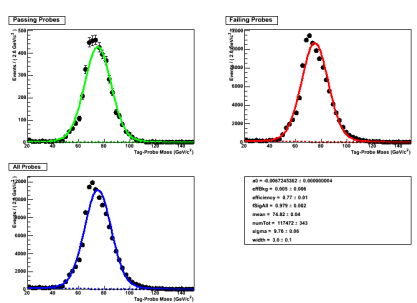

(c) $23-26 \mathrm{GeV}$

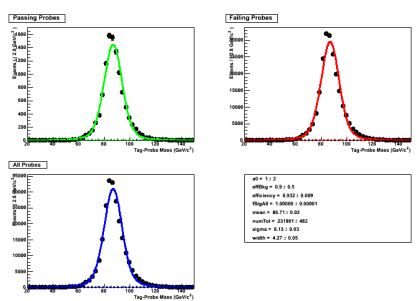

(f) $35-40 \mathrm{GeV}$
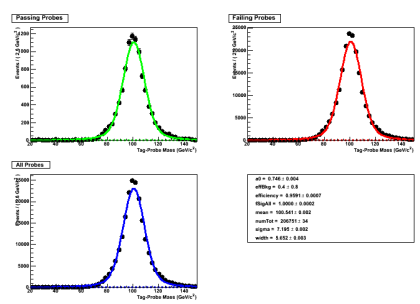

(i) $50-60 \mathrm{GeV}$

(j) 60-70 GeV

Figure C.4.: Data fitting results of tag-probe dimuon mass distributions for each $p_{T}$ bin with Tag and Probe applied for the trigger, HLT_L2DoubleMu23_NoVertex. Note that the range of each $p_{T}$ bin is indicated under each plot. 
back, the procedure could not be repeated due to the lack of statistics for small $\Delta R$ separation.

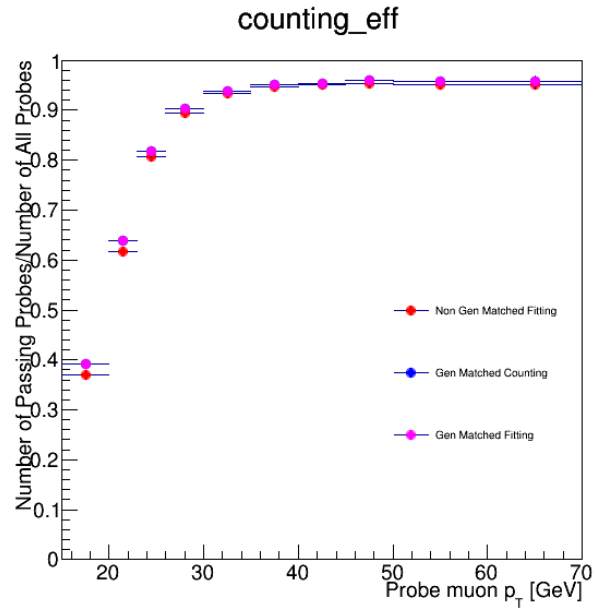

Figure C.5.: HLT_L2DoubleMu23_NoVertex trigger efficiency vs. $p_{T}$ graph. The results from fitting, counting and MC Truth fitting methods are compared. Note that Tag and Probe method is applied.

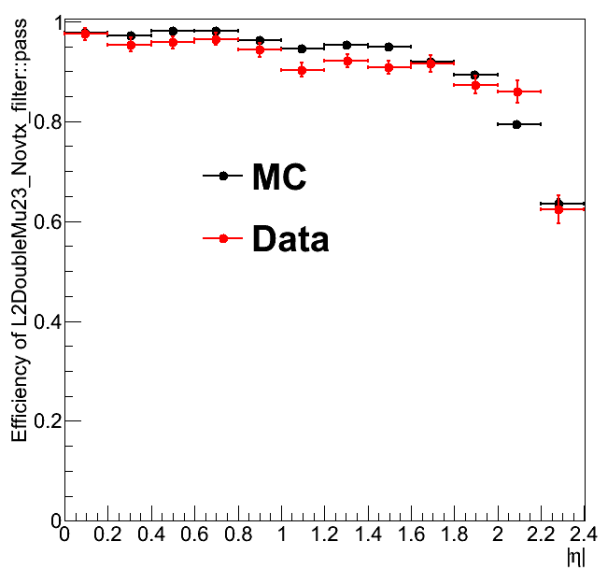

Figure C.6.: HLT_L2DoubleMu23_NoVertex trigger efficiency vs. $|\eta|$ graph for both the simulation and data with Tag and Probe applied. 


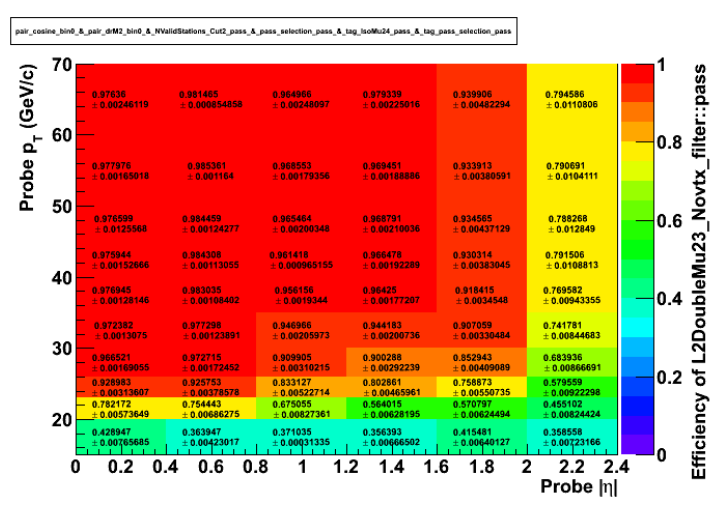

Figure C.7.: HLT_L2DoubleMu23_NoVertex trigger efficiency distribution on the $p_{T}$ and $|\eta|$ plane for MC with Tag and Probe applied.

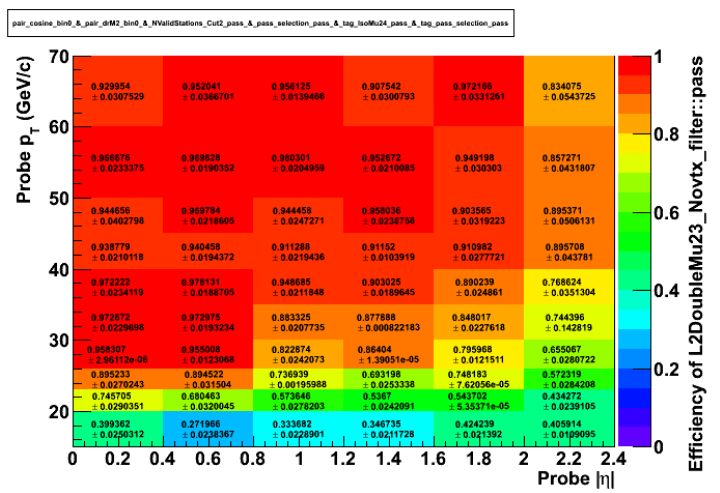

Figure C.8.: HLT_L2DoubleMu23_NoVertex trigger efficiency distribution on the $p_{T}$ and $|\eta|$ plane for data with Tag and Probe applied. 


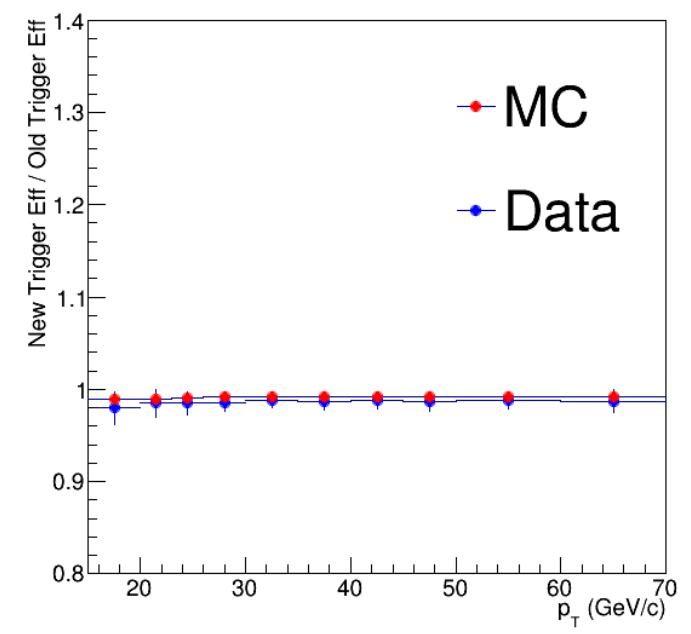

Figure C.9.: The ratio of HLT_L2DoubleMu23_NoVertex_2Cha_Angle2p5 trigger efficiency to HLT_L2DoubleMu23_NoVertex trigger efficiency vs. $p_{T}$ graph for both data and the simulation.

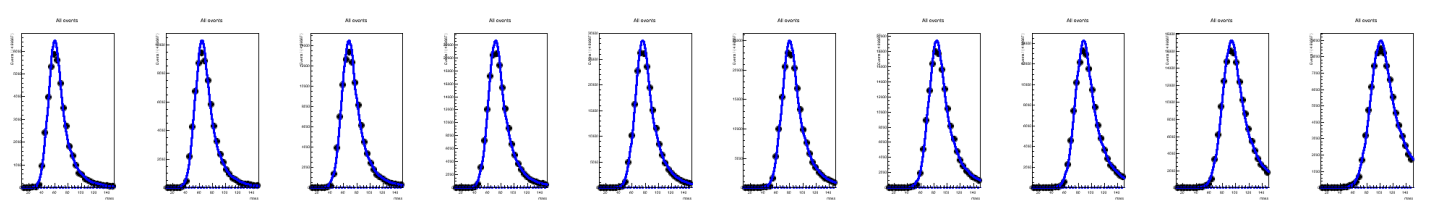

(a) Two RSA muons matched to HLT_L2DoubleMu23_NoVertex trigger

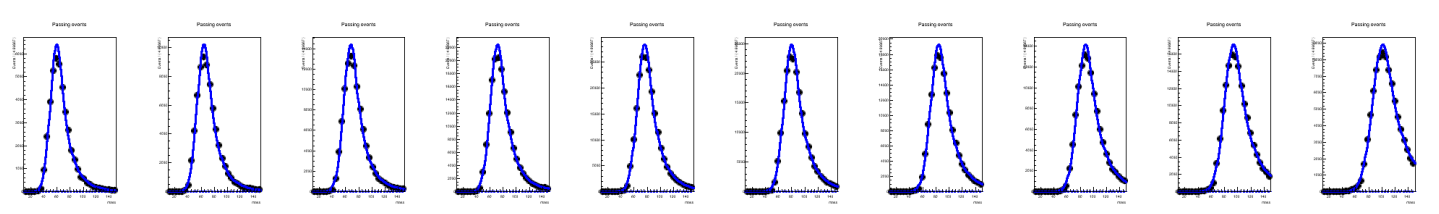

(b) Two RSA muons matched to both HLT_L2DoubleMu23_NoVertex and HLT_L2DoubleMu23_NoVertex_2Cha_Angle2p5 triggers

Figure C.10.: MC fitting results of dimuon mass distributions. The ratio of the integrals of the two curves in each $p_{T}$ bin gives the fraction of dimuons matched to HLT_L2DoubleMu23_NoVertex_2Cha_Angle2p5 trigger given that HLT_L2DoubleMu23_NoVertex is fired. 


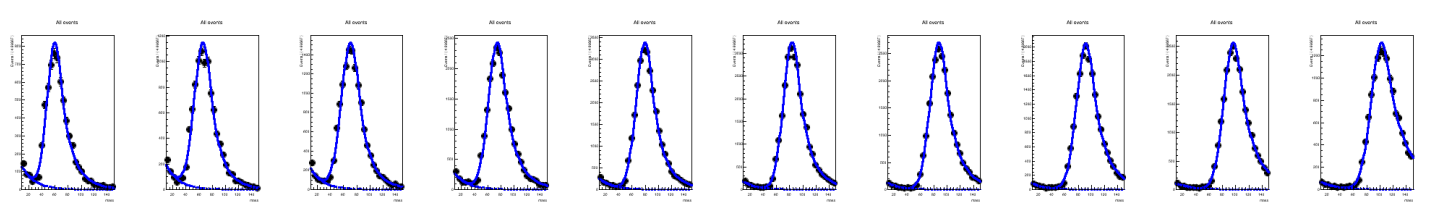

(a) Two RSA muons matched to HLT_L2DoubleMu23_NoVertex trigger
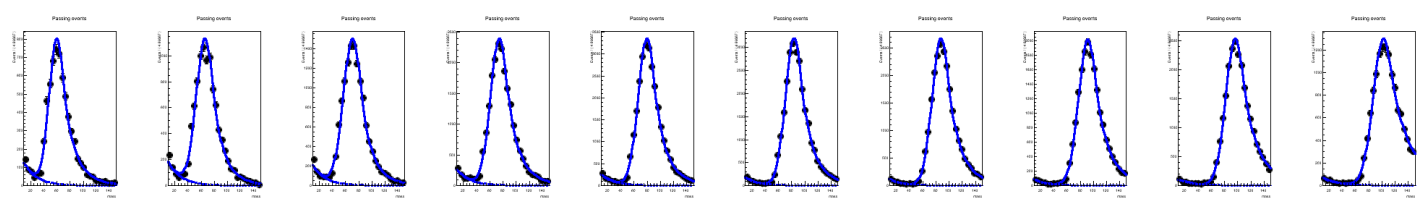

(b) Two RSA muons matched to both HLT_L2DoubleMu23_NoVertex and HLT_L2DoubleMu23_NoVertex_2Cha_Angle2p5 triggers

Figure C.11.: Data fitting results of dimuon mass distributions. The ratio of the integrals of the two curves in each $p_{T}$ bin gives the fraction of dimuons matched to HLT_L2DoubleMu23_NoVertex_2Cha_Angle2p5 trigger given that HLT_L2DoubleMu23_NoVertex is fired.

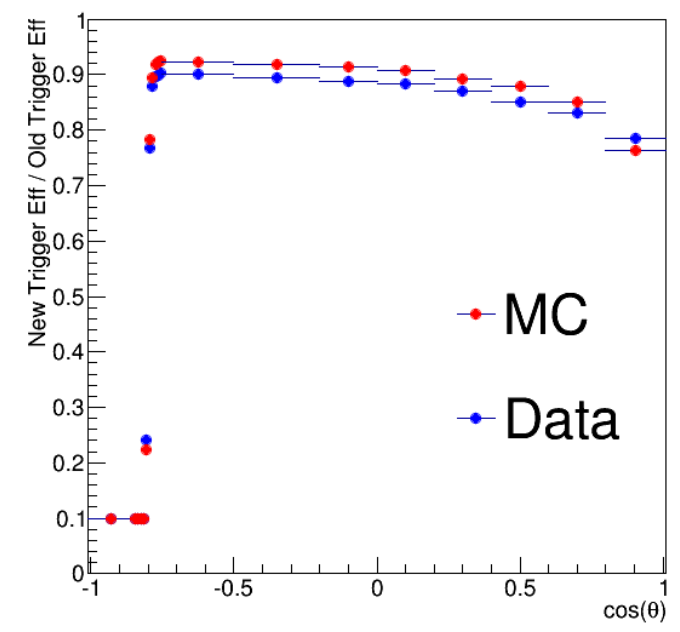

Figure C.12.: The ratio of HLT_L2DoubleMu23_NoVertex_2Cha_Angle2p5 trigger efficiency to HLT_L2DoubleMu23_NoVertex trigger efficiency vs. $\cos \theta$. Note that $\theta$ is the $3 \mathrm{D}$ angle between the two muons. 


\section{APPENDIX D. AGREEMENT BETWEEN DATA AND THE BACKGROUND SIMULATION}

Though it is already discussed in Chapter 4.3 that the simulation does not account for the expected cosmic background in the data, a reasonable agreement between the background MC samples and data is found. The plots from Figure D.1 to Figure D.5 are obtained by applying the full selection criteria except the cuts on the $\left|d_{0}\right| / \sigma_{d}$ and $L_{x y} / \sigma_{L_{x y}}$, tracker track rejection and the one plotted. They show that the agreement between data and simulation is good for all analyzed variables. Some discrepancies are

present for low $\cos (\alpha)$ and $\Delta R$ values that are excluded from the analysis. Additional discrepancies are present in the low mass region. Furthermore, MC dimuon mass resolution is also different than that of data. However, the detailed study presented in Appendix E shows that the analysis is significantly insensitive to the $p_{\mathrm{T}}$ resolution of the RSA muons.

Therefore, even though there are minor discrepancies between data and the simulation, they do not play an effective role within the scope of the analysis. We utilize the simulation only to compute the efficiencies of the signal samples. The comparison to the background $\mathrm{MC}$ is only used to assess whether the simulation is modelling correctly the variable distributions. We estimate the expected background directly from the data. Note also that this check is employed without unblinding the analysis. That is, only the agreement in the control region is examined. 

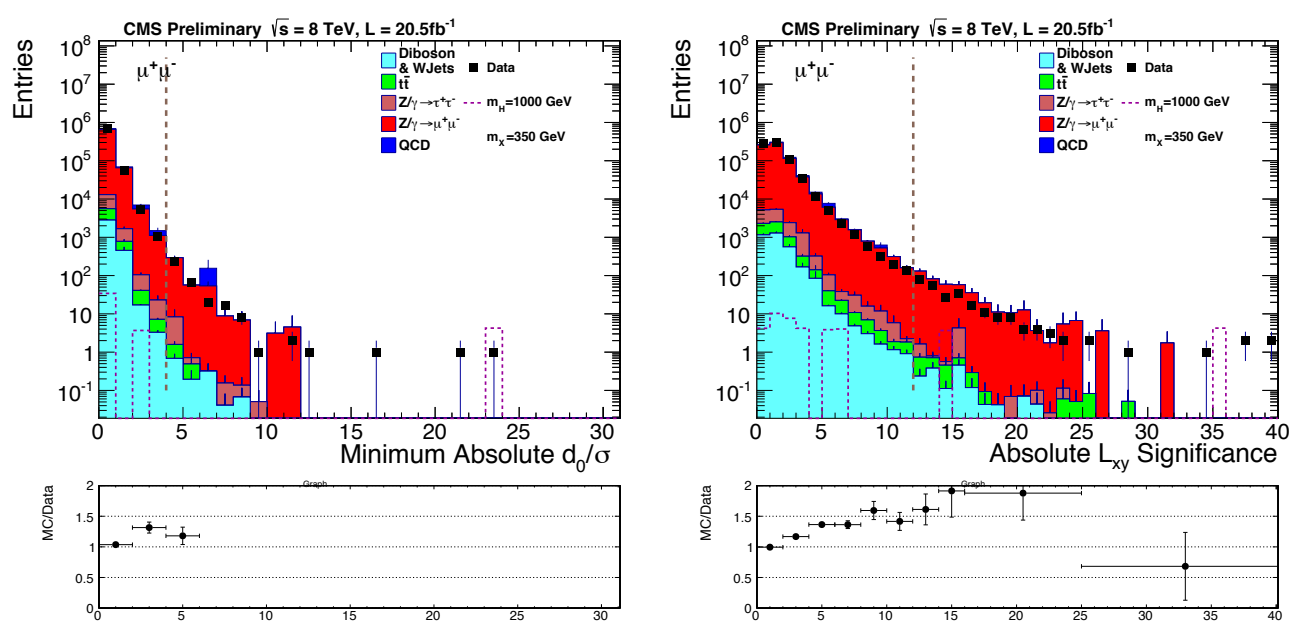

Figure D.1.: (Left) Distribution of the minimum absolute transverse impact parameter significance of the two muons for the dimuon candidates passing the selection. (Right) Distribution of the transverse decay length significance of the dimuon candidates passing the selection. The selection here is defined as the full analysis selection except $\left|d_{0}\right| / \sigma_{d}, L_{x y} / \sigma_{L_{x y}}$ and track rejection cuts in the control region. In addition to the selection defined, no cut is applied to the parameter shown in the plots. 

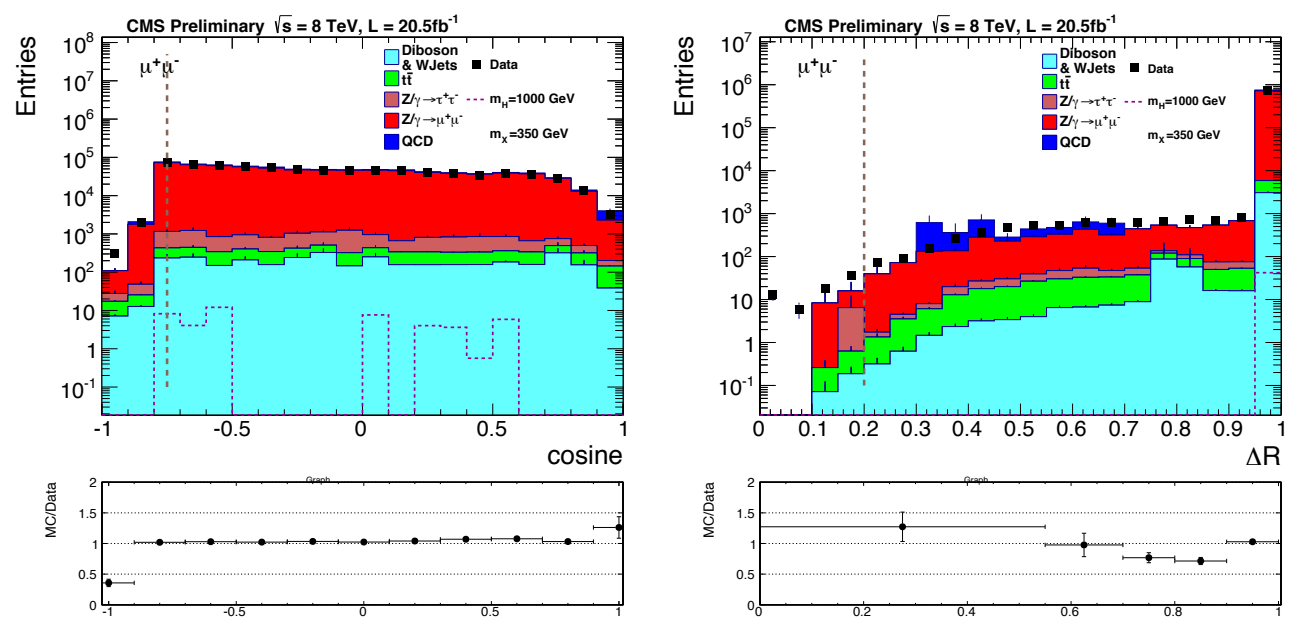

Figure D.2.: (Left) Distribution of the cosine of the 3D opening angle, $\cos (\alpha)$, between the two muons for the dimuon candidates passing the selection. (Right) Distribution of $\Delta R$ separation between the two muons for the dimuon candidates passing the selection.
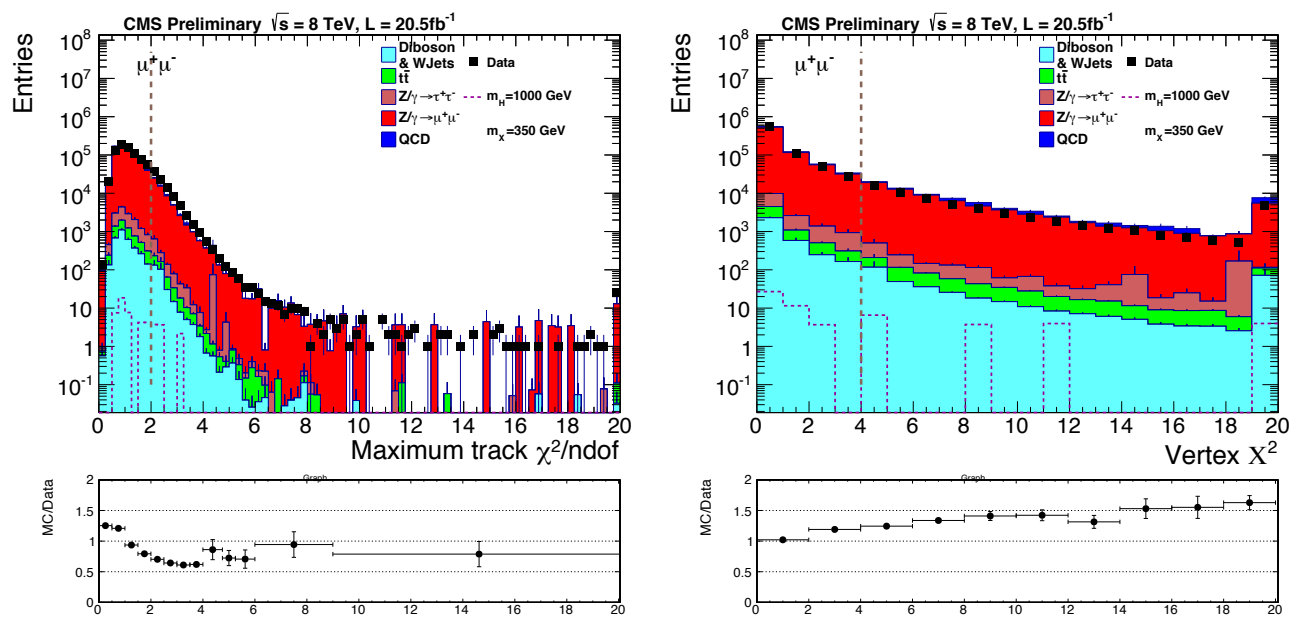

Figure D.3.: (Left) Distribution of the maximum normalized track $\chi^{2}$ of the two muons for the dimuon candidates passing the selection. (Right) Distribution of the normalized vertex $\chi^{2}$ of the dimuon candidates passing the selection. 

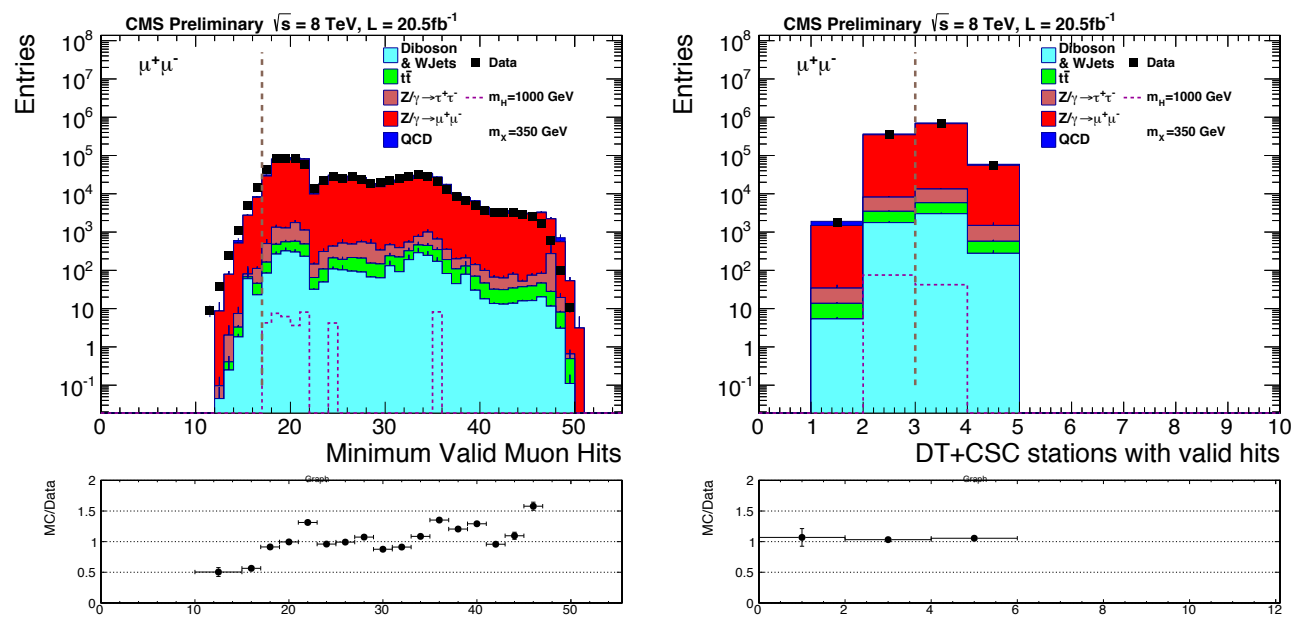

Figure D.4.: (Left) Distribution of the minimum number of valid muon hits of the two muons for the dimuon candidates passing the selection. (Right) Distribution of the minimum number of valid muon stations of the two muons for the dimuon candidates passing the selection.

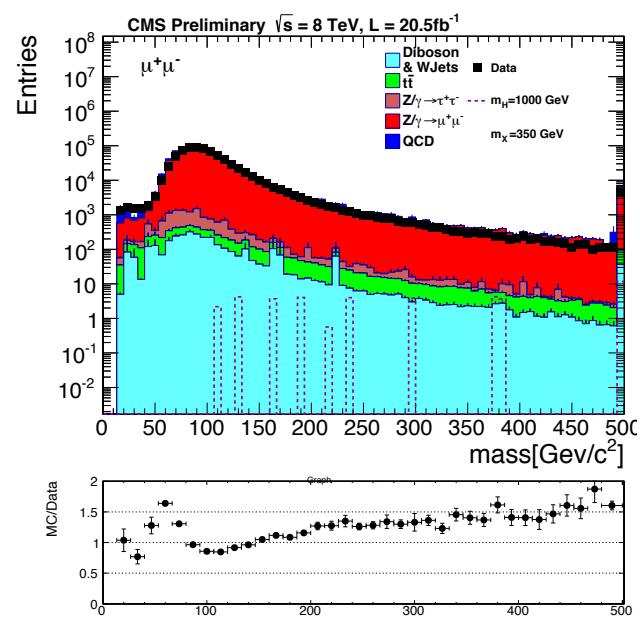

Figure D.5.: Distribution of the mass for the dimuon candidates passing the selection. 


\section{APPENDIX E. EFFECT OF THE RSA MUON $p_{\mathrm{T}}$ RESOLUTION ON THE} ANALYSIS

As shown in Appendix A, RSA muons have a much lower $p_{\mathrm{T}}$ resolution compared to the tracker muons. In this section, we examine whether an additional systematic uncertainty on this should be assigned given that the analysis selection has two cuts that directly depend on the $p_{\mathrm{T}}$ measurement.

The muons are required to have $p_{\mathrm{T}}$ of at least $23 \mathrm{GeV} / c$ at trigger level. In the offline analysis, we select the muons that have $p_{\mathrm{T}}$ of at least $26 \mathrm{GeV} / c$ to ensure that the trigger has a good efficiency. In addition, the minimum dimuon mass should be $15 \mathrm{GeV} / c^{2}$ to discard the dimuons consistent with coming from $\mathrm{J} / \psi$ and $\Upsilon$ decays and $\gamma$ conversions. The effect is expected to be negligible because loose selection criteria are applied to the muon transverse momentum and the dimuon mass. However, we performed a check to determine how much the analysis is affected by the $p_{\mathrm{T}}$ resolution of the RSA muons quantitatively.

Dimuon mass distribution is obtained for data and the background simulation by applying the full selection except the tracker track rejection and lifetime cuts, as given in Figure E.1. The figure shows that the two distributions are peaked in different

positions and that they have slightly different resonance widths. $p_{\mathrm{T}}$ resolution wise, data is not perfectly described by the simulation.

To quantify this discrepancy between the simulation and data, the distributions are fitted by Crystal Ball function due to the asymmetric long tail as presented in Figure E.2. Mean values of the two fit functions are found to be within the uncertainties of each other and the difference in these mean values between data and $\mathrm{MC}$ can be expressed by a relative difference of $1.13 \%$. Then, $p_{\mathrm{T}}$ of each muon in the simulation is increased by this factor in order to test how much the background MC dimuon mass distribution is shifted. As it can be seen in Figure E.3, this operation 
does not shift MC dimuon mass distribution enough to overlap with that of data. Furthermore, the signal selection efficiencies remain unchanged after this rescaling.

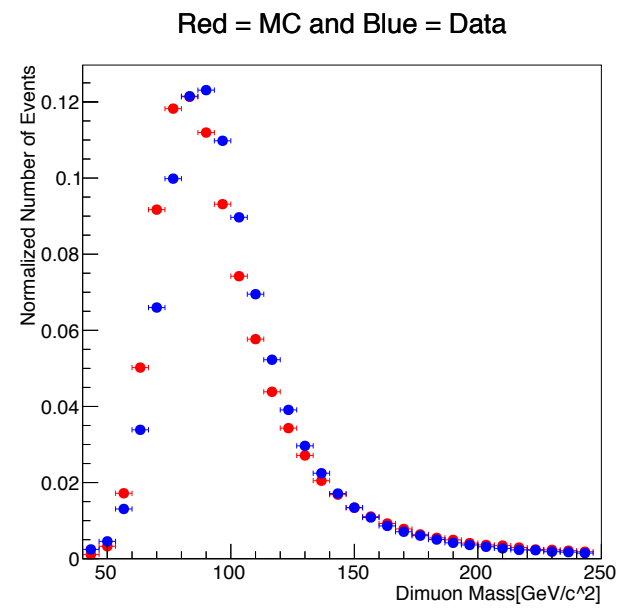

Figure E.1.: Dimuon mass distribution for both background MC, shown in red, and data, in blue. Note that the number of events is normalized.
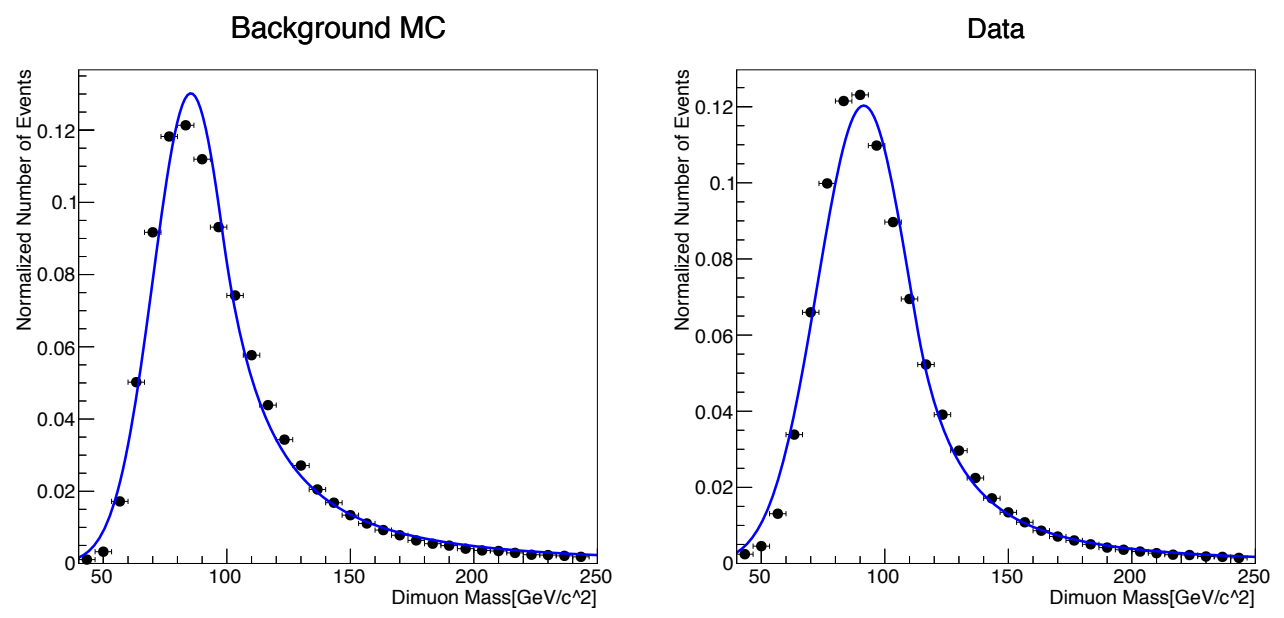

Figure E.2.: Dimuon mass distribution for background MC (left) and data (right) fitted by Crystal Ball function. Note that the number of events is normalized. 


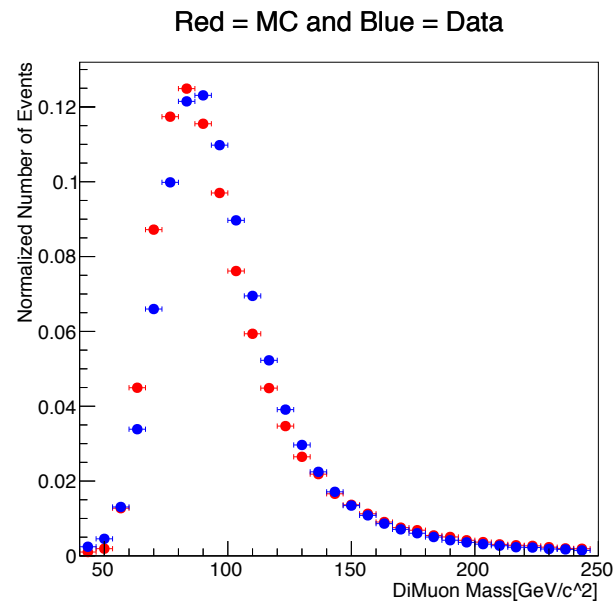

Figure E.3.: Dimuon mass distribution for both rescaled background MC, shown in red, and data, in blue. $p_{\mathrm{T}}$ is increased by a factor of $1.13 \%$ for each simulated muon. The dimuon masses are recomputed. Note that the number of events is normalized.

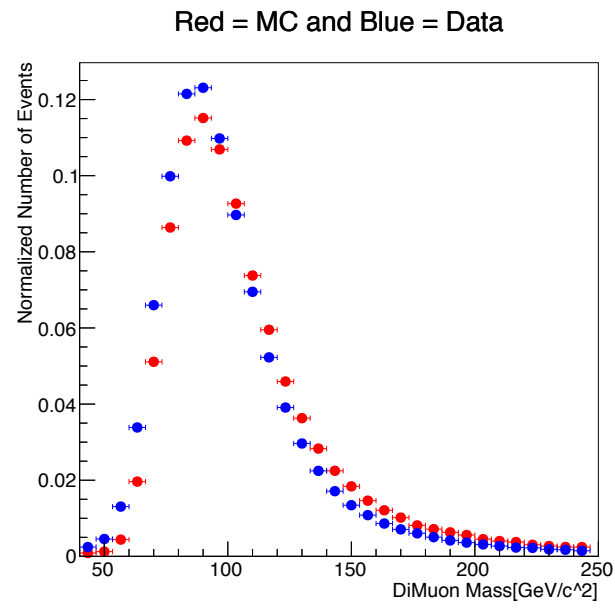

Figure E.4.: Dimuon mass distribution for both rescaled background MC, shown in red, and data, in blue. $p_{\mathrm{T}}$ is increased by a factor of $10 \%$ for each simulated muon. The dimuon masses are recomputed. Note that the number of events is normalized. 
In order to assess to what extent the analysis is sensitive to $p_{\mathrm{T}}$ resolution, the $p_{\mathrm{T}}$ of each muon in the background and signal simulations is increased by an unreasonably big factor of 10\%. Then, the procedure is repeated as previously. Figure E.4 indicates that the background MC dimuon mass distribution is shifted even beyond that of data under such scaling. However, no significant variation in the signal efficiencies of all mass points is observed. Therefore, assigning an additional systematic uncertainty on $p_{\mathrm{T}}$ resolution of the RSA muons is not found to be necessary since our analysis is significantly insensitive to it. 


\section{APPENDIX F. $L_{X Y}$ RESOLUTION OF RSA MUONS FOR PROMPT DECAYS}

To evaluate the resolution of the transverse decay length, we used simulated $\mathrm{Z} \rightarrow$ $\mu^{+} \mu^{-}$events that have a reasonable agreement with data as given in Appendix D. Since $\mathrm{Z}$ bosons decay promptly, there is no genuine secondary vertex. One might be led to think that this could yield a half gaussian distribution for $L_{x y}$ peaking at zero owing to the resolution effect. Nonetheless, it will be shown why it is not the case.

Note that $L_{x y}$ is the distance between the primary and secondary vertices, hence, always positive:

$$
L_{x y}=\sqrt{\left(x_{P V}-x_{S V}\right)^{2}+\left(y_{P V}-y_{S V}\right)^{2}}
$$

where $x_{P V}-x_{S V}$ and $y_{P V}-y_{S V}$ are the distance between primary and secondary vertices in $\mathrm{x}$ and $\mathrm{y}$ directions.

If the secondary vertex is assigned symmetrically around the primary vertex, then, the vertex distances in $\mathrm{x}$ and $\mathrm{y}$ directions are expected to have a gaussian distribution as justified in Figure F.1. That is, the secondary vertex is positioned around the primary vertex symmetrically for the prompt decays. However, same symmetry can not be met by $L_{x y}$ since it is square root of the square sum of the two gaussian distributions. Even though Figure F.2 might, at first glance, infer that the secondary vertex positions are distributed unevenly around the primary vertex, there is actually two fold symmetry coming from both $\mathrm{x}$ and $\mathrm{y}$ directions. So, it is not straightforward to derive the resolution on $L_{x y}$ in the first place.

$L_{x y}$ resolution is computed by a rough estimate on the resolution of $x_{P V}-x_{S V}$. Figure F.3 shows that the gaussian fit applied is reasonable. The sigma value of the gaussian fit is about $3 \mathrm{~cm}$. Then, if two resolution effects are combined, one could roughly estimate the resolution of $L_{x y}$ as $3 \sqrt{2} \approx 4.2 \mathrm{~cm}$. This result is valid only for prompt dimuon decays. 


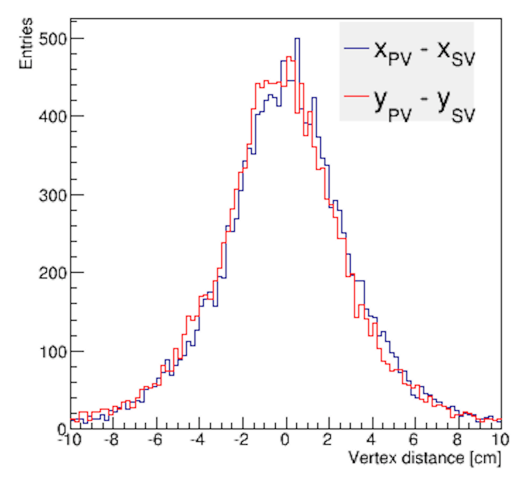

Figure F.1.: Distribution of the distance between primary and secondary vertices in $\mathrm{x}$ and y planes for the simulated $\mathrm{Z} \rightarrow \mu^{+} \mu^{-}$events. The shapes look gaussian indicating the symmetry of the decay.

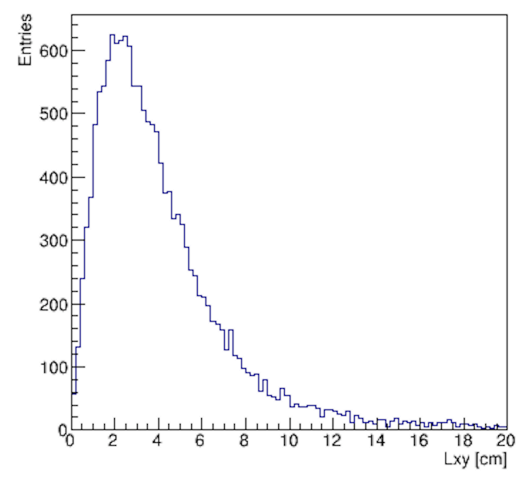

Figure F.2.: Distribution of the transverse decay length for the simulated $\mathrm{Z} \rightarrow \mu^{+} \mu^{-}$ events. Since it is square root of the square sum of the two gaussian distributions, the resolution of $L_{x y}$ can not be trivially extracted from this shape. 


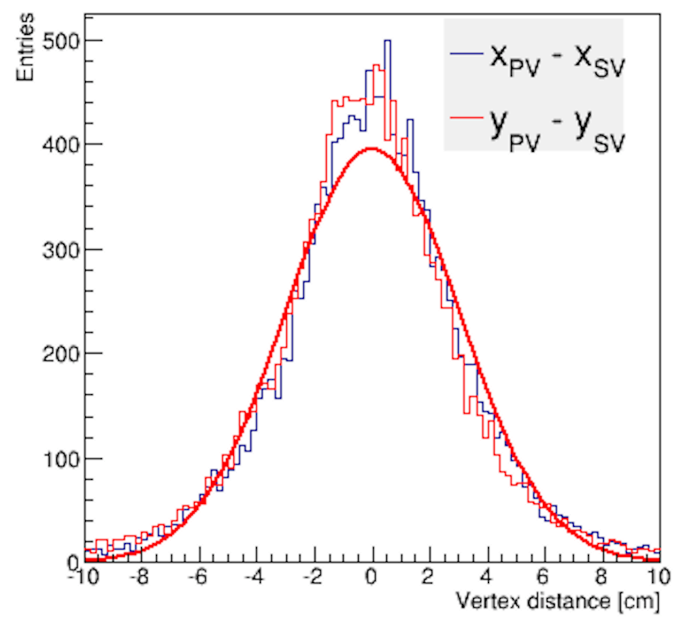

Figure F.3.: Distribution of the distance between primary and secondary vertices in $\mathrm{x}$ and $\mathrm{y}$ planes for the simulated $\mathrm{Z} \rightarrow \mu^{+} \mu^{-}$events. Bold red curve is the gaussian fit applied to one of the two. The sigma value of the fit function is 3.00918 . 


\section{APPENDIX G. SECONDARY VERTEX RECONSTRUCTION EFFICIENCY}

The algorithm used to fit a common secondary vertex from two tracks is the KalmanVertexFitter [21]. The default implementation in the CMSSW release used in this analysis contains a cutoff of the size of the silicon tracker. We extended the cutoff to the approximate beginning of the muon chambers in order to recover efficiency for particles decaying outside the tracker. The following changes were made to RecoVertex/VertexTools/src/SequentialVertexFitter.cc:

- TrackerBoundsRadius was changed from 112 to 500,

- TrackerBoundsHalfLength was changed from 273.5 to 1000.

To verify the performance of the algorithm for decays outside its previously intended range, we study the secondary vertex reconstruction efficiency in signal MC. We also perform an additional cross check by using cosmic data and comparing them with results obtained on cosmic simulation.

\section{G.1 Secondary vertex reconstruction efficiency in signal MC}

We compute the secondary vertex reconstruction efficiency on signal MC. The reference sample used is $H \rightarrow X X$ with $M_{H^{0}}=400 \mathrm{GeV} / c^{2}, M_{X}=50 \mathrm{GeV} / c^{2}$ and $c \tau=800 \mathrm{~cm}$. We consider the generated $L_{x y}$ as the position of the secondary vertex. The primary vertex is reconstructed using tracker tracks and it is measured with an accuracy of the order of 100 microns or less. The resolution on $L_{x y}$ is dominated by the resolution on the secondary vertex position. A good agreement between measured and generated $L_{x y}$ indicates a good agreement between the generated and reconstructed position of the secondary vertex in the transverse plane. In all the results shown in this section we do not apply any explicit trigger requirement. Figure G.1 shows the correlation of $L_{x y}$, its error and its significance with the generator level $L_{x y}$. A good 
correlation is found for the $L_{x y}$ value up to 4 meters. The $L_{x y}$ error shows a cutoff at about 3.4 meters where it assumes a default value of $100 \mathrm{~cm}$. As a consequence, the $L_{x y}$ significance is generally below the cut of 12 used in the analysis for this region. However, the effective range of the analysis is limited by the trigger efficiency to approximately 2.5 meters and this behavior does not affect the results. It should be certainly improved in case the analysis sensitivity is extended to even longer decay length values in the future.
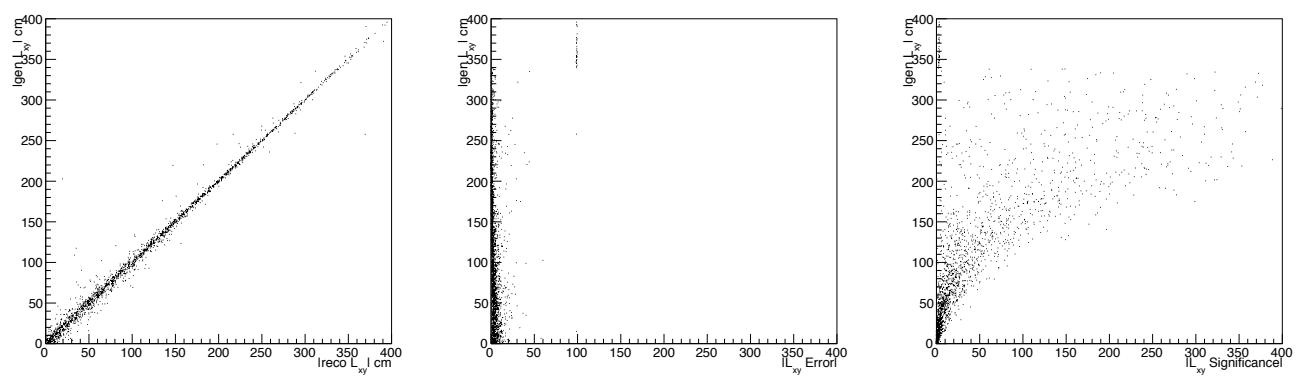

Figure G.1.: Correlation of the reconstructed $L x y$ (left), $L_{x y}$ error (center), and $L_{x y}$ significance (right) with the generated $L_{x y}$. The $L_{x y}$ error defaults to a value around $100 \mathrm{~cm}$ after approximately 3.4 meters. This is outside the effective region of the analysis that is limited to approximately 2.5 meters by the trigger efficiency.

To evaluate the secondary vertex reconstruction efficiency we consider all generated long-lived particles decaying to dimuons where the two muons are matched to RSA muons and among these events (denominator), we consider the cases where a secondary vertex has been successfully reconstructed (numerator). The efficiency is computed as the ratio of the numerator and the denominator terms. The distributions of the number of all generated long-lived particles decaying to dimuons and of those passing the numerator and denominator selections are shown in Figure G.2. Figure G.3 shows the secondary vertex reconstruction efficiency. No significant dependence on $L x y$ is observed and we do not assign any additional systematic uncertainty. 


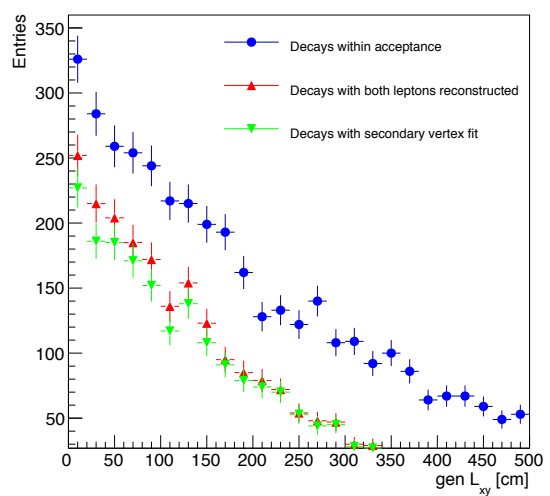

Figure G.2.: Distribution of the generated $L_{x y}$ for all generated long-lived particles decaying to muons (blue), for those that have both muons reconstructed (red), and for the ones where a secondary vertex is also successfully reconstructed (green).

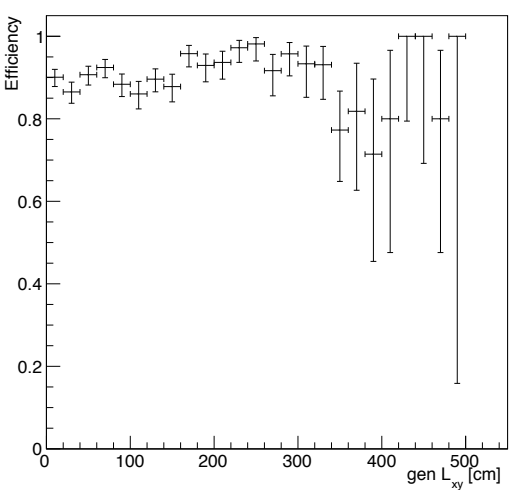

Figure G.3.: Efficiency to reconstruct a secondary vertex in signal MC events for $M_{H^{0}}=400 \mathrm{GeV} / c^{2}, M_{X}=50 \mathrm{GeV} / c^{2}$ and an average lifetime of $800 \mathrm{~cm}$. 


\section{G.2 Secondary vertex reconstruction efficiency in cosmics data and sim- ulation}

To evaluate the efficiency for data as a function of the displacement of the tracks with respect to the expected beamspot, we use cosmic data and cosmic simulation. The datasets used are described in Chapter 6.3 with the difference that for the data we utilize cosmics collected in the "Run C" period instead of the CRAFT period.

In cosmic events there is no well defined $L_{x y}$ so it is not possible to express the efficiency as a function of this variable. The impact parameter of the tracks, however, is a good measurement of the displacement of the reconstructed secondary vertex. We therefore show the efficiency vs $d_{0}$ and we compare the results obtained in data and simulation. The result cannot be directly compared to the efficiency obtained in the previous section but it provides a cross check of the agreement between the secondary vertex reconstruction efficiencies in data and simulation. Note, however, that if the track is completely straight, the position of the secondary vertex is arbitrary since the two RSA tracks, when extrapolated, will coincide (ideally). To reduce this effect we require that the two RSA muons are not completely back-to-back. We select events with only two reconstructed RSA muons with an angle between them, $\alpha$, such that $-0.95<\cos (\alpha)<0.90$. The upper bound is to avoid possible cases of duplicates where the two RSA tracks are actually reconstructed from a subset of the hits of the same half of the cosmic. The two muons are also required to be within the acceptance of the analysis $\left(p_{T}>26 \mathrm{GeV}\right.$ and $|\eta|<2$.). Among those events we select the ones where a secondary vertex was successfully reconstructed. The ratio of these two categories of events is a measurement of the (fake) secondary vertex reconstruction efficiency in the cosmic events. The distributions of number of events with two reconstructed RSA muons within the acceptance as a function $d_{0}$ of the highest $p_{T}$ track in the event and of those where a secondary vertex is reconstructed are shown in Figure G.4 for data and simulation. The resulting efficiencies are presented in Figure G.5. Good agreement is found between data and simulation and we do not 
assign any additional systematic uncertainty from secondary vertex reconstruction efficiency. The choice of the $d_{0}$ from the highest $p_{T}$ RSA muon in the event is arbitrary. However, the highest $p_{T}$ RSA muon is expected to be the better measured half of the cosmic and thus have a better resolution on $d_{0}$.
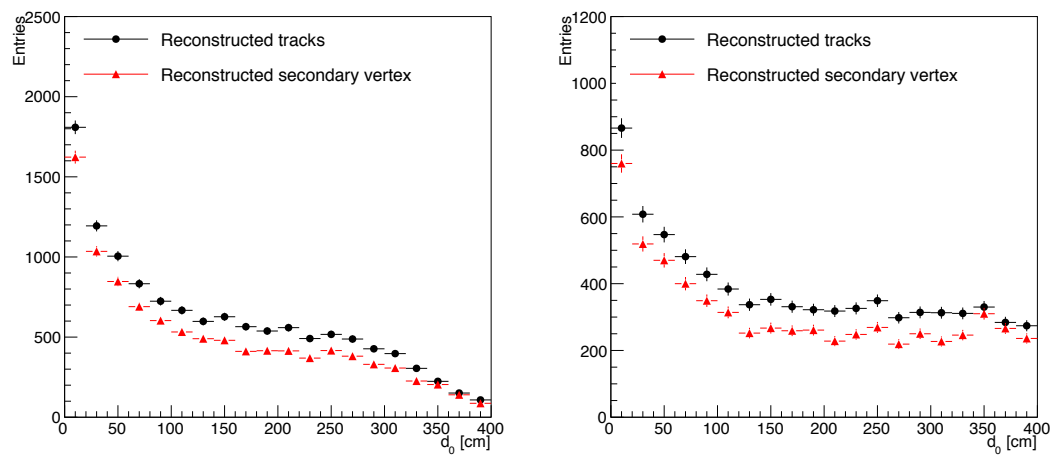

Figure G.4.: Distribution of the number of cosmic events in data (left) and simulation (right) with two reconstructed RSA muons within the acceptance (black) and with the additional requirement of a reconstructed secondary vertex (red) as a function of the $d_{0}$ of the muon with the highest $p_{T}$.

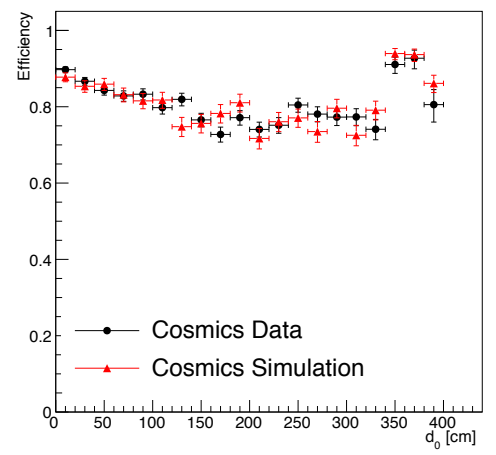

Figure G.5.: Efficiency to reconstruct a (fake) secondary vertex in cosmic data (black) and simulation (red) as a function of the $d_{0}$ of the highest $p_{T}$ RSA muon in the event. 


\section{APPENDIX H. ALTERNATIVE BACKGROUND SYSTEMATICS ESTIMATE}

As an additional method to the principal one to derive a systematic uncertainty on the background estimate presented in Chapter 5.2, the $L_{x y} / \sigma_{L_{x y}}$ tail-cumulative distribution for the data is obtained after applying all the remaining cuts in the control region. Only 3 events are left in the control region after the full selection except the $L_{x y} / \sigma_{L_{x y}}$ cut is applied. Given the low statistics, an unbinned fit is performed on the $L_{x y} / \sigma_{L_{x y}}$ cumulative distribution with an exponential function. The evaluation of the fit function at $L_{x y} / \sigma_{L_{x y}}=12$, the cut value, gives another estimate on the number of expected background events in the signal region, that is $0.23 \pm 0.24$. Secondly, the procedure can also be iterated for the $\left|d_{0}\right| / \sigma_{d}$ cumulative distribution. In this case, there are only 2 events left if the complete selection is applied except the $\left|d_{0}\right| / \sigma_{d}$ cut. Figure H.1 demonstrates both tail-cumulative distributions. Fitting the $\left|d_{0}\right| / \sigma_{d}$ cumulative distribution yields an estimation of zero background events in the signal region. Note that the errors in the fit results obtained by this method do not take into account that the data points in these cumulative distributions are correlated to each other. Even though the two results are consistent with each other, having very few statistics necessitates a more reliable method. 

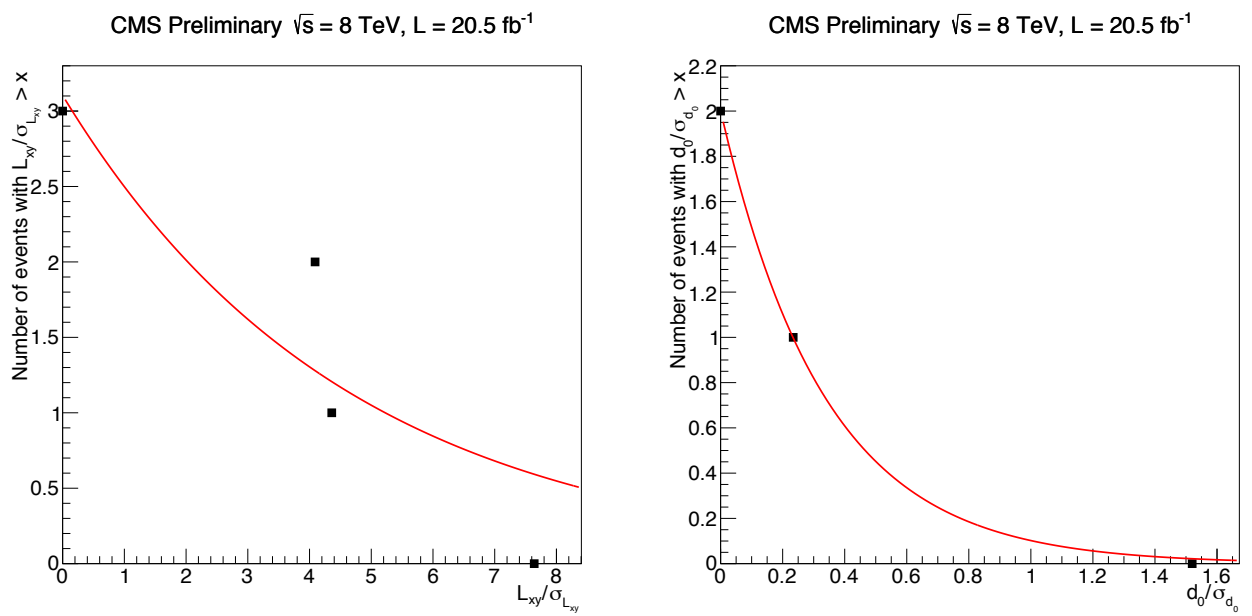

Figure H.1.: (Left) $L_{x y} / \sigma_{L_{x y}}$ cumulative distribution as all the remaining cuts are applied. (Right) $\left|d_{0}\right| / \sigma_{d}$ cumulative distribution. The most conservative estimate of background comes from the left one, $0.23 \pm 0.24$, suggesting that the systematic uncertainty on the number of estimated background can be set as high as 0.47 . Note that the red curves represent the exponential unbinned fit functions. 